SF 199

. A14 A5

1914

Copy 1
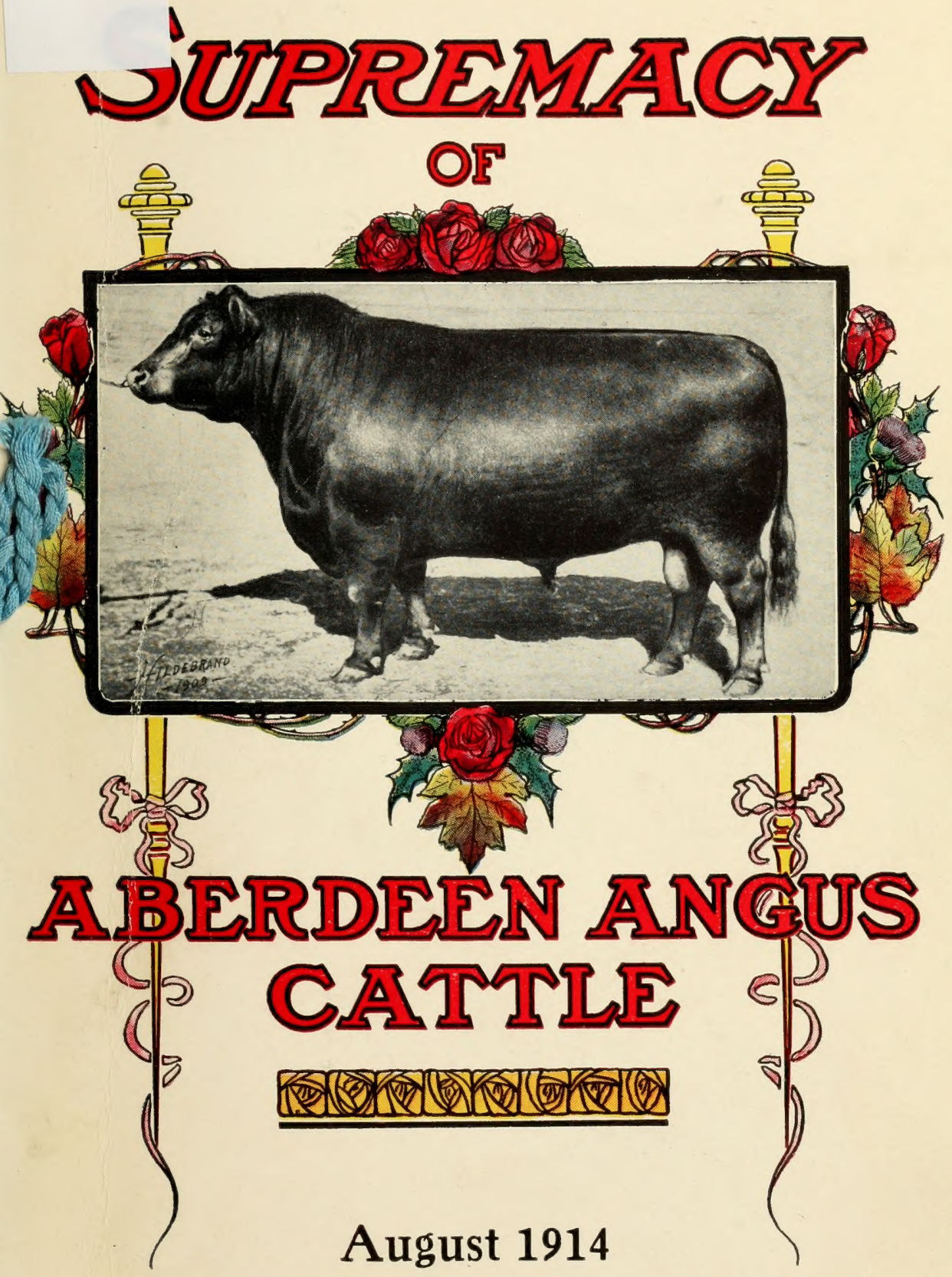



\title{
Supremacy of
}

\section{Aberdeen-Angus Cattle}

Results of Leading Fat Stock Shows of

Great Britain and America

Third Edition

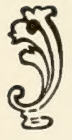

EDITED BY CHAS. GRAY, SECRETARY

\author{
PUBLISHED BY
}

AMERICAN ABERDEEN-ANGUS BREEDERS' ASSOCIATION

817 EXCHANGE AVENUE, CHICAGO 



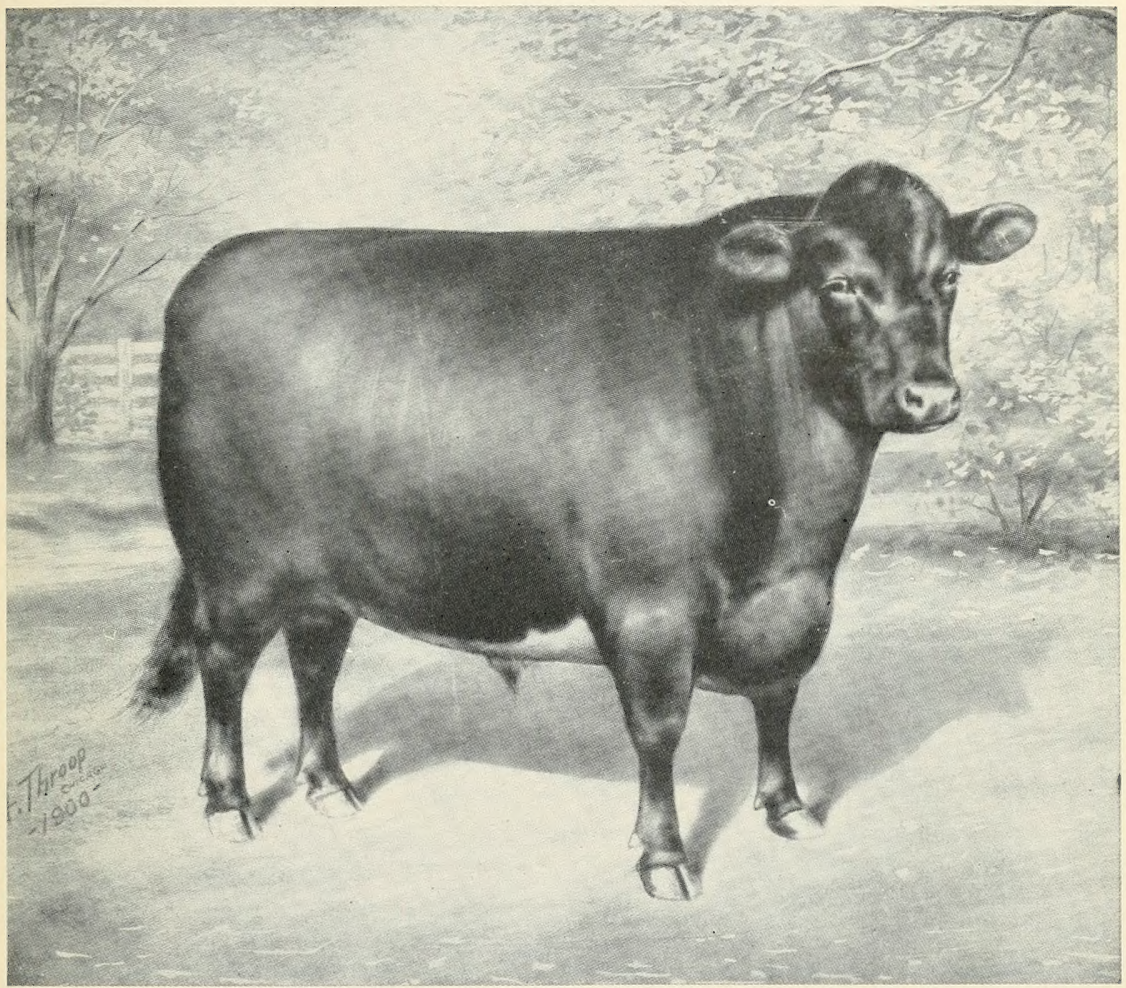

ADVANCE?

An Aberdeen-Angus Product of Illinois

First International Live Stock Exposition Grand Champion Fat Steer over all Breads. Bred, Fed and Exhibited by Stanley R. Pierce, Sold at World's Record Pric,
$\$ 1.50$ Per Pound by Bowles \& Co. 


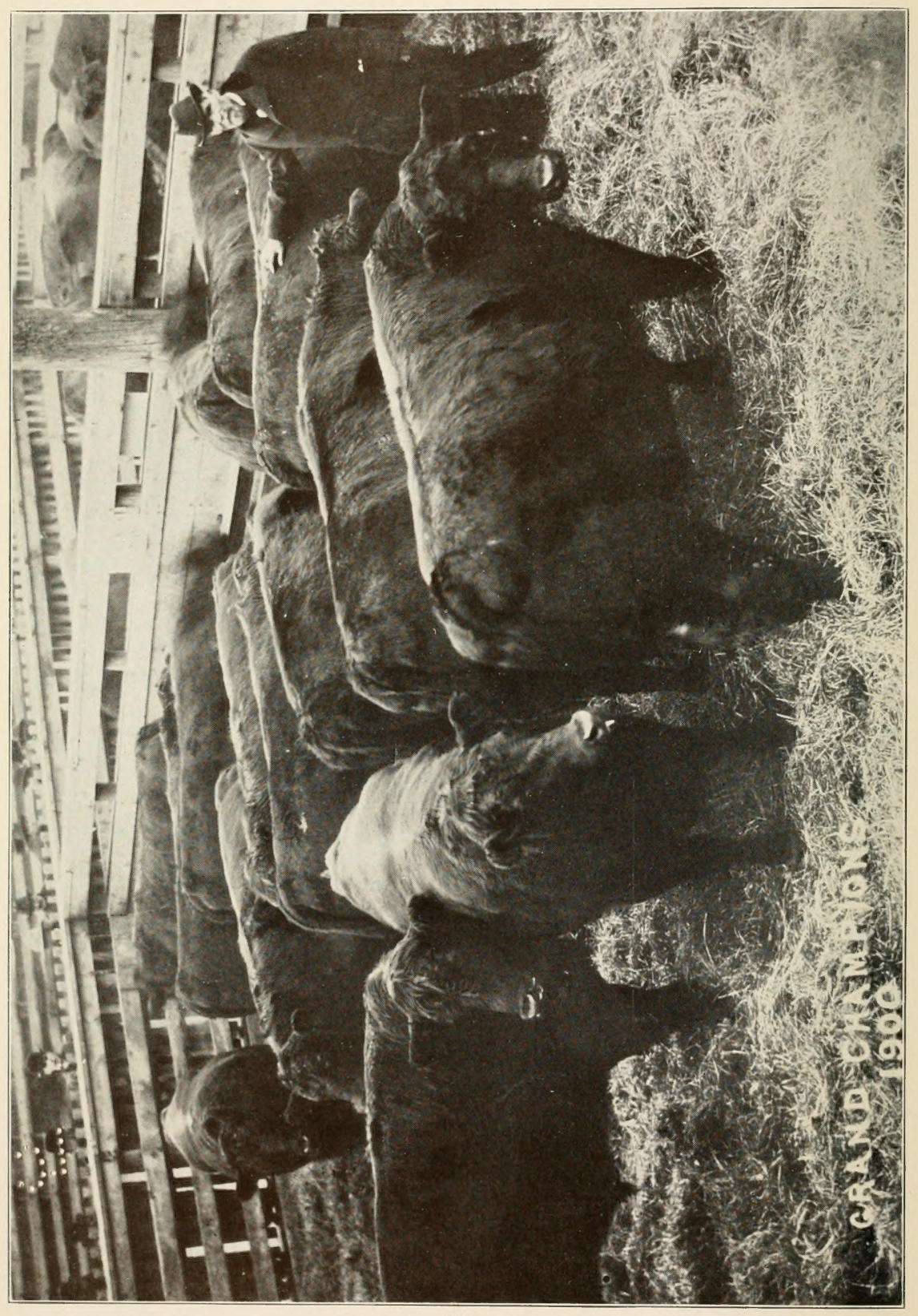

Photo by Courtesy of Clay, Robinson \& Co. AN ABERDEEN-ANGUS PRODUCT OF ILLINOIS

Grand Champion Carload of Fat Steers at 1900 International Live Stock Exposition. Sold at $15 \frac{1}{2} \mathrm{C}$ per $\mathrm{lb}$, by Clay, Robinson \& Co. Fed and Exhibited by Late Hon. L. H. Kerrick. 


\section{CONTENTS}

\section{Awards of American Fat Stock Shows}

Pages

Introduction

Relation of Pure-Bred Aberdeen-Angus to the Cattle Industry...................

Retrospective and Prospective of Beef Production (by .I. E. Poole).............

Table of Awards and Summary of Single Fat Animal Awards at International.

Table of Awards and Summary of Fat Carload Lot Awards at International.

Table of Awards and summary of International Carcass Contest...............

Aberdeen-Angus Cattle on the Range (by George Findlay)............
Pages

Aberdeen-Angus in Feed Lot (by A. W

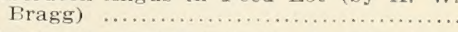

Table of Awards of St, Joseph show, Iowa State and Inter-state Fairs, Western Stock Show, Fort Worth and Canadian Shows

Table of Comparative Average Prices Obtained at Auctions by Breeds from

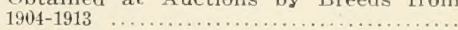
Why Aberdeen-Angus are Popular....... Aberdeen-Angus Mature Early........... Progressive Agrieulture

Recapitulation of American Shows......

\section{8}

\section{Awards of British Fat Stock Shows}

Introduction

Aberdeen-Angus and Their Crosses (by J. R. Barclay)

Summary of Smithfield Show Awards...

63

Smithfield Carcass Awards and Summary Summary of Birmingham Show Awards Summary of Edinburgh, Aberdeen and

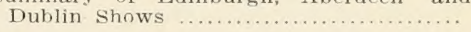

\section{Illustrations}

Advance, 1900 International Grand Cham-

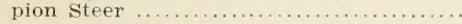
International 1900 Grand Champion Fat

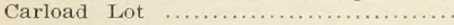

Glencarnock Victor, 1912 International

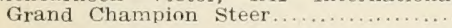

Glencarnock Victor 2d. 1913 International

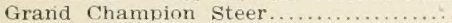

Doctor Gwinn, 1909 International Cham-

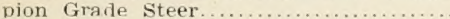

Victor, 1910 International Grand Cham-

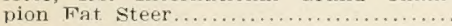

King Ellsworth, 1909 International Grand

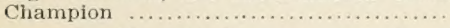

Shamrock 2d, 1910 International Grand

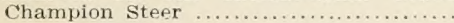

Clear Lake Jute 2d, 1904 International

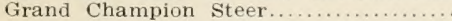

Fyvie Knight, 1908 International Grand

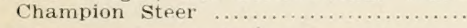
1913 International Grand Champion Fat

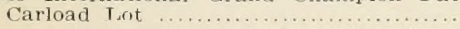

Pittsburg Grand Champion Fat Carload

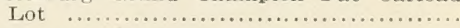

International Grand Champion Carcass

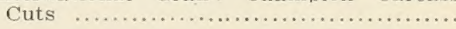

International 1908 Champion Fat Carload

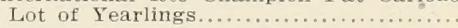
Fort Worth, 1910 Grand Champion Fat

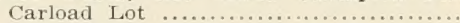

Chuck and Round from Carcass of Exilio, Grand Champion............... 44

1906 International Grand Champion Fat Carload Lot ........................

1906 American Royal Grand Champion Carload Lot ....................... 50

Western Stock Show, 1910 Grand Charspion Feeder Carload Lot.............., , 54 Western Stock Show, 1908 Grand Chasin-? pion Fat Carload Lot................, 54

Violet $3 \mathrm{~d}$ of Congash................ 64

Beauty of Welbeck, 1913 Grand Champion at Smithfield and Birmingham.......... 64 


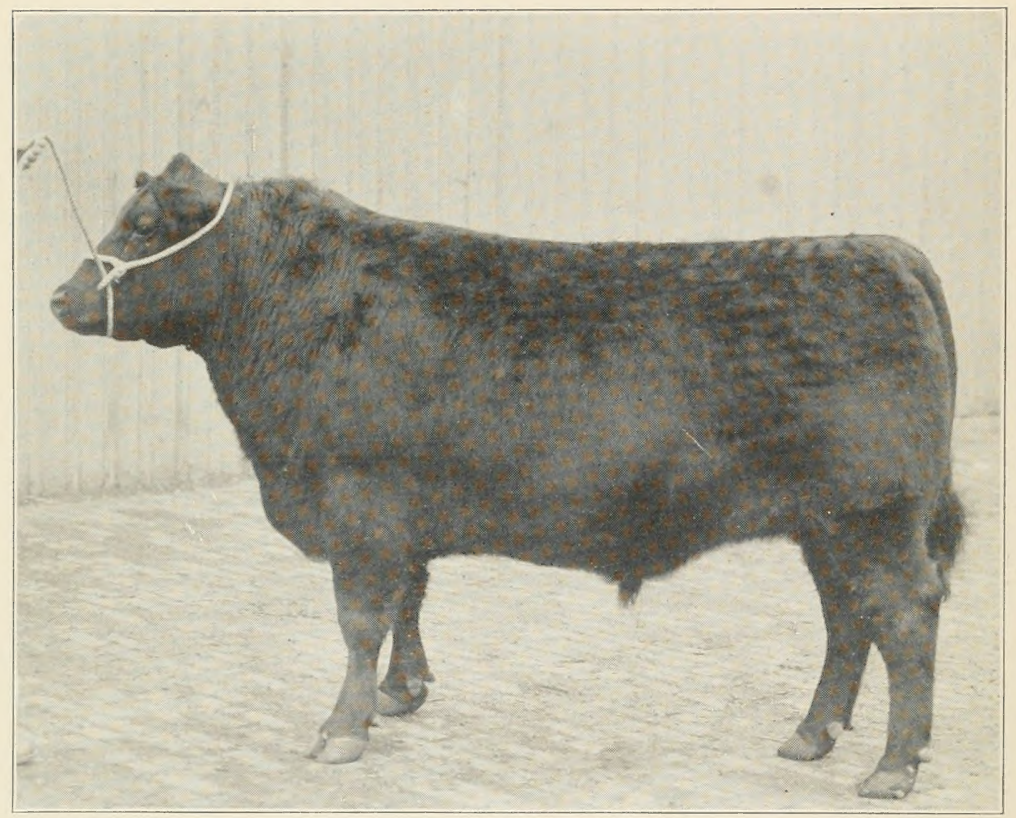

GLENCARNOCK VICTOR

1912 International Jive Stock Exposition, Chicago, Grand Champion Steer over Al! Breeds. Bred, Fed and Exhibited by J. D. MeGregor, Brandon, Manitoba, Canada.

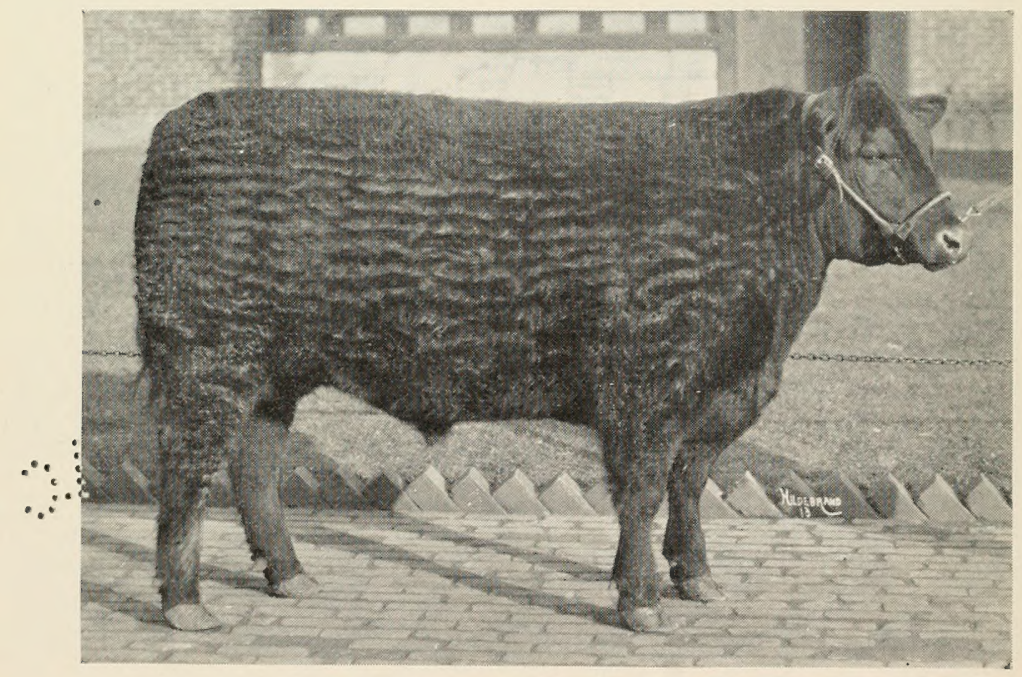

GLENCARNOCK VICTOR $2 \mathrm{~d}$

1913 Intcrnational Live Stock Exposition, Chicago, Grand ('hampion Stere Over All

Breeds. Bred, Fed and Exhibited by J. D. McGregor, Brandon, Manitoba, Canada. 


\section{Introduction}

The first and second editions of "Supremacy of Aberdeen-Angus Cattle" issued by the American Aberdeen-Angus Breeders' Association in 1909 and 1910 met with such universal favor among Aberdeen-Angus breeders as well as breeders and feeders of other breeds of live stcck and those interested in the fat stock shows and expositions, that the large editions which were printed are exhausted. At a meeting of the Board of Directors of the American Aberdeen-Angus Breeders' Association a motion was passed instructing the Secretary to revise the pamphlet, incorporate the up-to-date show results and print a large enough edition to supply all demands. Many features of interest have been added in form of contributions from eminent authorities on the cattle industry. The illustrations have been improved and new ones added in order to make the pamphlet more practica! and instructive. Much could be written in form of elaboration on the contents but the author feels sure the concise form will be preferred and appreciated by the majority of readers.

The chief object of this pamphlet is for the purpose of setting forth the comparative merits of the leading beef breeds in Gruat Britain and America. To treat this subject scientifically and practically in an exhaustive manner would require much more time than the average man of this busy age can afford, especially in America, thus only the essence of the facts that enter into the results of the past decade of British and American Shows and Expositions has been used.

So much of a general nature has been written on the merits of beef breeds that the theories or speculations constitute a mass of material that is like a mountain of bewilderment to the average cattleman. Elaboration for explanation of a theory is necessary; however, right at this point every practical and scientific cattleman should get fixed in mind the definition of the terms practical, scientific, theory and hypothesis.

$\mathrm{Al}$ ' arguments of the merits of all the beef breeds should be based on practical or scientific foundations. America is a country of science with practice, thus no up-to-date American should lag in the ruts of theory or listen to dreams that belong to the category of hypothesis.

To avoid generalization and at the same time present the true merits of the Aberdeen-Angus breed of cattle, the results of the leading Live Stock Shows and Expositions have been used, where the leading beef breeds of cattle have been in direct competition in Great Britain and America. The past fifteen years is considered the best criterion of the beef cattle industry in America; therefore the results obtained from such a period are without question a true index of the merits of the rival beef breeds. 
It is conceded by all leading cattle authorities that practical and scientific production of beef has reached a higher degree of excellence in Great Britain and America than in any other countries of the world. It is further conceded that the marvelous results obtained have been accomplished chiefly by the object-lessons presented at the great Live Stock Shows of Great Britain and America, such as the International Live Stock Exposition, Chicago, Illinois; American Royal Live Stock Show, Kansas City, Missouri; Inter-State Show, St. Joseph, Missouri; W/estern Stock Show, Denver, Colorado; National Stock Show, Fort Worth, Texas; Winter Fairs, Guelph, Ontario, and Brandon, Manitoba, Canada, etc., in America; London Smithfield Show and Birmingham Show, in England; Edinburgh and Aberdeen Shows, in Scotland, and Dublin Show, Ireland.

The data has been compiled with the utmost care from the official records of the various shows. The author fee!s greatly indebted to the Secretaries and Superintendents of British and American Shows, Breeder's Gazette, Banffshire Journal, Country Gentleman, James E. Poole, and George Findlay for the courtesy and assistance tendered in form of valuable contributions and illustrations.

\section{Relation of Pure-Bred Aberdeen-Angus to the Cattle Industry.}

Great as has been the progress in every department of agriculture during comparatively recent years, there is probably no problem that deserves more attention or confronts the American people more forcibly than the production of meat animals, especially beef animals, to furnish food for the population of this great American nation, which is continually increasing at a rate nothing short of marvelous.

The British people have been consistent beef-eaters for many generations, and it is conceded by leadir 8 authorities that the British people have progressed more rapidly during the time they have used meat as one of their chief foods than during any other period covering the same number of years. Many eminent writers and philosophers attribute the strength and success of the British people to the use of beef. The people of the United States are destined to be great because they possess much of the best blood of all the leading nations of the world and because the Americans have used meat more liberally as one of the chief foods of their diet than any other people.

For these sound reasons no one should give heed to people with a fanatic turn of mind who instigate boycotts on packers and producers of beef. Yellow journalism has done much to stir the equilibrium or steadiness of the American farmer. Nearly everything in the country has been organized except the farmer, and he is rapidly being educated in a manner that will mean organization ultimately in a more gigantic form than anything that has yet been in evidence. When such a time arrives, the pure-bred cattle interests and their associations will be of such significance that they will be a power in this country in a measure that has scarcely been dreamed of by farmers, stockmen and those engaged in the cattle business. 
Some would-be authorities seem to be of the opinion that the pure-bred cattle business has reached its zenith. It only requires a little investigation, however, to enlighten or convince any one of such absurd ideas or vague conceptions of the pure-bred cattle industry.

There are scarcely fifty million beef animals in the United States and considerably less than half a million pure-bred living registered animals in use for the purpose of improving the quality of beef animals. In other words, there is only about one pure-bred animal to every one hundred grades and scrubs. A full realization of these facts should readily convince every breeder, stockman and farmer, as well as those interested in the cattle industry, that the pure-bred cattle business and the beef cattle Record Associations are on a sound, solid foundation, although mere! $y$ in their infancy.

It is not an uncommon thing to hear men of high positions in the sphere of the cattle industry question the merit of the use of pure-bred registered animals and the Record Associations. Those who have any doubt about the value of pure blood need only make a general survey of the improvement that has been wrought on the native and range cattle of our country during the past twenty-fivc or thirty years, or since pure blood has been permanently established.

The great continual success of the fat stock shows of Careat Fritain are chiefly duc to the systematic use of pure blood and the phenomenal success of the fat stock shows of the United States and Canada can be who'ly attributed to the fertile brains of the pure-bred breeders who have labored as pioneers, as it were, without the assistance of Government legislation or assistance from leading capitalists.

This immense cattle industry which has grown to such proportions and spread over such vast territories of this country has its sheet-anchor or foundation in the use of pure blood, and furthermore it is dependent on the use of pure-bred animals in order to maintain the perfection it has already reached. There is nothing that will do more to attain greater perfection in beef cattle than the use of more purebred Aberdeen-Angus, or some of the other recognized beef breeds. If the pure-bred cattle Associations and their members were to forsake their noble work, such action would be a ca'amity to the cattle industry of this country and the noted specimens of beef animals would soon deteriorate to the old-time dogies and scrubs. The pure-bred cattle Associations and the systematic methods of keeping pure-bred animals are here to stay as long as our country continues to be a leader in the important branch of agriculture known as live stock farming.

Much more cculd be added" in form of elaboration to show the intrinsic valuc and relation the pure-bred cattie have to other cattle and the industry in general; however, it is not deemed necessary. Lecause the writer is fully convinced it is only the question of a short time until the real significance and power of the purebred beef cattle interests will be recognized and appreciated in a much higher degree by everyone interested in cattle, the Government, and the people of this country. 


\title{
Retrospective and Prospective of Beef Production
}

\author{
By JAMES E. POOLE, Editor Chicago Live Stock $W$ orld and Assoctate Editor \\ of Breeder's Gazette.
}

Beef scarcity was a much derided theory less than three years ago. Consested markets were attributeci to generous production instead of the real causc, liquidation. For more than a decade the common impulse among producers was to get out of the cattle business. A minority either actuated by foresight or favorably situated for carrying on breeding operations retained cow herds and when scarcity emerged from the theoretical stage to become a fact found themselves in possession of remunerative property. Beef production declined at a period when consumption was increasing by leaps and bounds and almost at the inception of the year 1914. Despite opening of the markets of the United States to the beef producers of the world a few months previcusly ithe fact became apparent that the much advertised Antipodean plentitude was not equal to restoration of the cheap beef era. Combelt feeders were confronted with the impossibility of replenishing feed lots save at prices that were prohibitive of profit and the steer. man west of the Missouri River faced extinction as a production factor. Within a few months the cattle breeder found his business established on a remunerative basis, with every prospect of a continuance of that condition. Forecasters of this evolution, accustomed to ridicu'e, commanded respect and public concern over the natural beef supply acauired emphasis.

Hardly had the first leaf been torn from the ca'endar of the year 1914 when values of pure-bred cattle were enhanced on somewhat pyrotechnical fashion and in this appreciation the Aberdeen-Angus were in the van. Sale records tell the story adequately. Bulls that a few years previous'y were scont to the shambles encountered inquiry from every nook and cranny of the country. Breeders, tempted by prices that would have been regarded as impossible a few years before, parted with their bovine possessions only to discnver that they had blundered and in many cases reinstatement was accomplished at considerable added expense. Many breeders refused to price cattle, sale rings were thronged by anxious hidders, bull buyers with unfilled orders rode highways and byways in quest of suitable material and the fact that a 'amentable shortage of both pure-bred and commercial cattle existed became patent. Even a period of commercial and industrial depression failed to repress the quest for material necessary to recuperate the industry. Had the purchasing power of the industrial population of the country been normal during the first six months of the current year, a furore in pure-bred cattle trade would have been inevitable.

How seriously reduced commercial catt'e volume became at this juncture is indicated by market supply statistics. During the first half of 1914 six principal western markets, Chicago, Kansas City, Omaha, St. Louis. St. Joseph, and Sioux 
City, received approximately but 2,825,000 cattle, against 3,215,000 the previous year, 3,230,000 in 1912, and 3,616,000 in 1911, at which period supply was normal. The figures show a supply shrinkage in three years at these markets alone o! 775,000 cattle, while at primary recciving points of lesser importance the decrease was proportionately as great. At some western markets vast s'aughtering p?ants were thrown into disuse, butchering gangs were reduced 60 per cent and beef consumption in the United States dropped to the smallest volume since the present method of distribution was developed. Mexico and Canada, advertised scurces of supply plentitude failed, even when tariff bars were thrown down to afiord maierial relief and considerable quantilies of Australian and Argentine bee! disappeared into the ravenous maw of the market without mitigating the condition of the consumer. Coincident'y caitle values advanced all crer the civilized world and protest at beef cost became as vociferous at Buenos Aires and Brisbane as at Chicago and New York. Decline of the catt'e industry in the United States had world wide influence.

But what has happened is an oft-lold story, and the public is more interested in supply prospects than in reminisence. The position that cattle growing in contradistinction to feeding is impossible on high-priced cornbelt land is no longer tenable, its fallacy having been abundantly disproved and the fact is equal'y demcnstrable that if the people of the United States are to coninue the heaviest beef consumers in the world, domestic production must increase. Reinstatement of the breeding industry in a commercial sense has undoubtedly been retarded by skepticism concerning the future, but Argentine and Australian surplus production is obviously incapable of nullifying shortage elsewhere and despite congressional action in placing dressed meats on the free list, 1914 cattle prices have averaged at the highest levels on record. Even the doubing ones are beginning to realize that foreign competition is not the serious factor anticipated. American consumers are fastidious and such beef as Aberdeen-Angus yearlings or two-year-old steers are convertible into wil! never experience serious competition from Antpodean pastures. Those who realized this fact are getting remunerative results while the timid ones have nothing to sell.

The fact will not be disputed that on this North American continent more beef making material is going to waste than at any time since the bison was evicted from the trans- Missouri region. An \$8@8.50 market for the few Aberdeen-Angus yearlings and calves reaching the central markets demonstrates how profitably this waste could be utilized. Feed plentitude is no mere local condition. Montana has been carrying a hay accumulation of several seasons, in Texas the prairie dog has been allowed to take possession of millions of acres of good grazing ground. East of the Mississippi and south of the Ohio is a vast area fitted by nature for cattle production, and in the cornbelt the silo has practically doubled production capacity. Only reinstatement of breeding herds is neccessary to insure an adequate national supp'y of beef, but the very nature of that task renders an excess impossible. The 
average western settler, cornbelt farmer or southern planter is neither adapted to cattle raising nor in the mood for that undertaking, consequently overproduction is not to be feared. For an indefinite period the breeder will pocket most of the profit resulting from cattle production.

Aberdeen-Angus cattle are mote than holding their own in the somewhat tardy reinstatement of the business. During the past year they have been distributed over a wide area. Western growers no 'onger permit cattle to rutle for a living, and where the haystack decorates the landscape, the placid, weight-maing, thrifty "doddie" isk without competition. The Eastern South has been absorbing Aberdeen-Angus breeding stock, missionary work by the association in that quarter having been effective in facilitating the distribution process, and the buyer with a contract to secure a car load of bulls of serviceable age, has had a difficult task. As continuance of high prices arouses interest in beef cattle production, demand for purebred cattle will expand, and the annual increase will be contracted before weaning time. Breeding operations south of the Ohio River will undoubtedly expand now that the tick scourge is being repressed and the cornbelt market rendered accessible for ca'ves bred in that region at all seasons.

Profitable beef production in the future means early maturity, and no yearling commands the attention of the buyer as readily as the one wearing a black coat. And, if the testimony of feeders is worth anything, an Aberdeen-Angus calf handles better, makes a better average gain, and responds more readily to the care essential to successful baby beef production than any other breed. This fact is fully attested by the celerity with which the stock cattle market is cleared of them. And when the feeder returns to market with finished product, no peddler's license is necessary to vend it.

Circumstances have created an opportunity. Those endowed with foresight to seize it are reaping a rich reward, but knocking is sti!l distinctly audible. Excess production of good cattle is as impossible as a glut of gold, and the hundred-dollar fat yearling, of either sex, will produce better net results than any other system of utilizing the soi' products of the Mississippi Valley, the western slope, the intermountain region or any other section of this North American continent where grow in abundance the grasses and grains on which the bovine species thrives. 


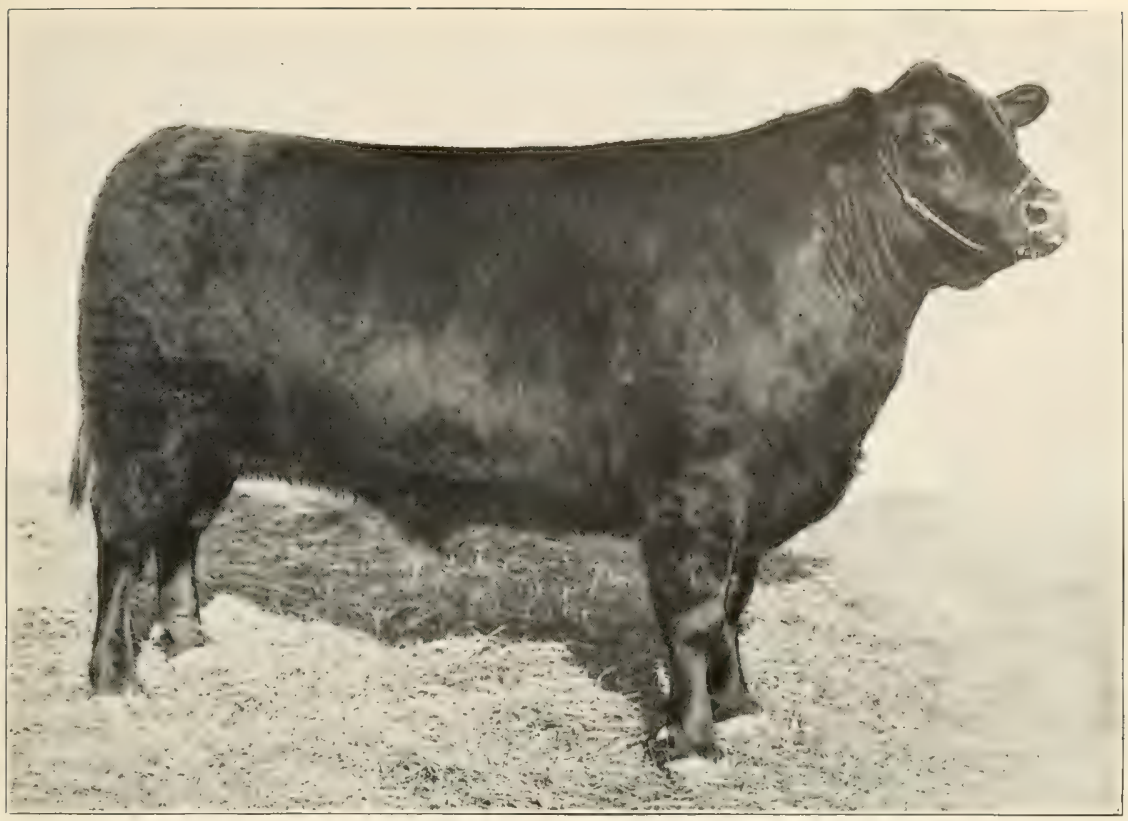

DOCTOR GWINN

An Aberdeen-Angus Product of Ohio.

1909 International Live Stock Exposition Champion Grade Steer over all Breeds. Fed and Exhibited by Mart L. MeCoy.

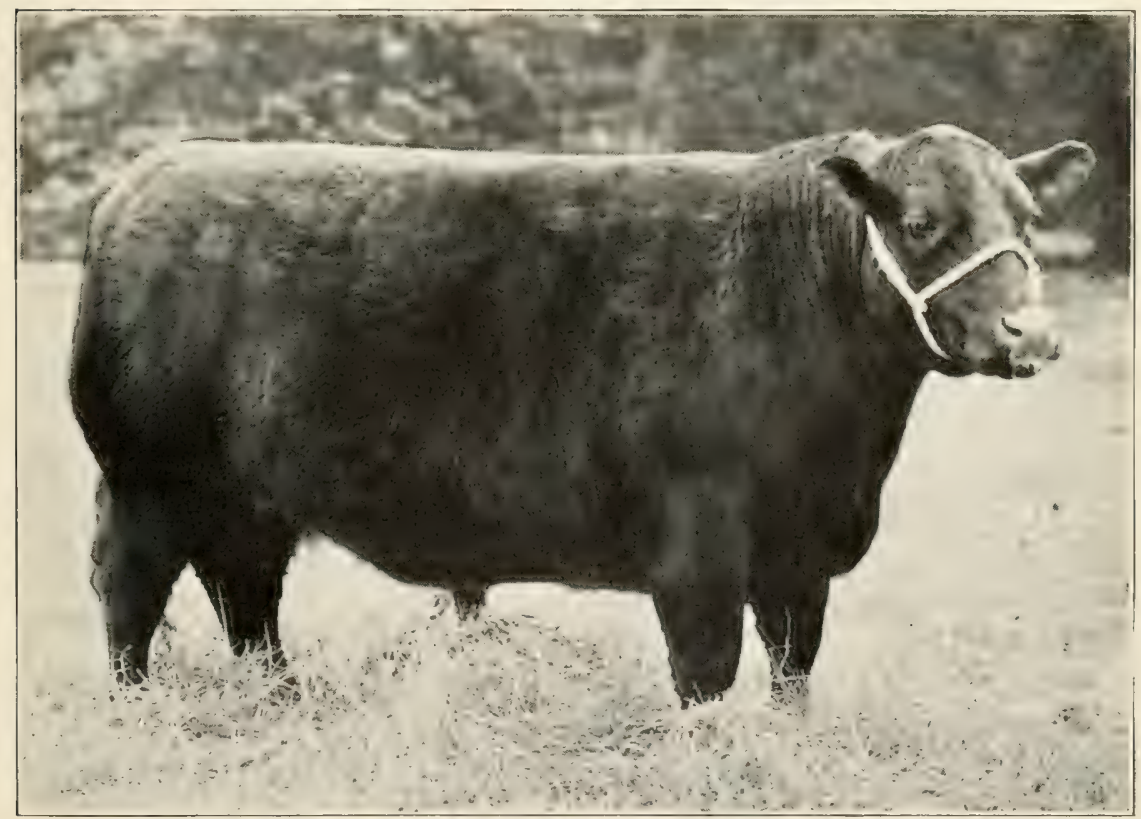

\section{VICTOR}

An Aberdeen-Angus Product of Iowa

1910 Grand Champion Fat Steer over all Breeds at National Feeders and Breeders' Show, Fort Worth, Texas. Bred, Fed and Exhibited by W. J. Miller. Also 1911 International Live Stock Exposition Grand Champion Steer over all Brceds. Fed and Exhibited by Iowa State College. 


\title{
Chicago \\ International Live Stock Exposition Awards
}

\section{Grades and Cross-Breds}

\author{
Competition Limited to Steers, Spayed or Marlin Heifers
}

TOTAL ENTRIES.

CLASS.

Aberdeen- Shorthorn Hereford Mixed Angus rating rating rating rating

33 several not shown: 2 years and under 3

$1234 \quad 1234$

1234

1234

48 several not shown: 1 year and under 2 .

0200

$\begin{array}{llll}0 & 0 & 0 & 0\end{array}$

$\begin{array}{llll}1 & 0 & 3 & 4\end{array}$

$\begin{array}{llll}0 & 0 & 0 & 0\end{array}$

25 several not shown: under I year.

$\begin{array}{llll}1 & 0 & 3 & 0\end{array}$

$\begin{array}{llll}0 & 0 & 0 & 4\end{array}$

$\begin{array}{llll}0 & 0 & 0 & 0\end{array}$

0 A 200

7 shown: herd.

$\begin{array}{llll}0 & 0 & 0 & 0\end{array}$

$\begin{array}{llll}0 & 0 & 0 & 0\end{array}$

$\begin{array}{llll}1 & 0 & 0 & 4\end{array}$

$0 \mathrm{~B} 2 \mathrm{c} 30$

A Hereford-Shorthorn, B Hereford-Holstein, C Aberdeen-Angus-Calloway.

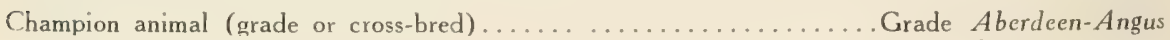

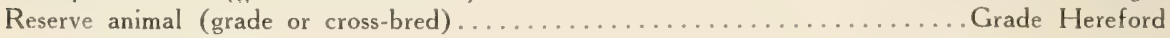

Champions by Ages (Pure-breds, Grades and Cross-breds Competing).

Champion animal 2 years old and under 3.

Reserve animal 2 years old and under 3

Champion animal 1 year old and under 2 .

Aberdeen-Angus

Reserve animal I year old and under 2 .

Hereford

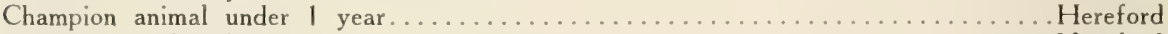

Reseive animal under I year

Hereford

Grand champion single animal of the show............................

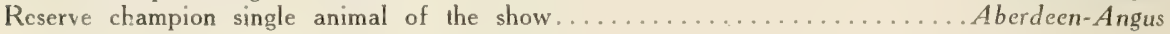

1901

TOTAL ENTRIES. CLASS.

Aberdeen- Shorthorn Hereford Mixed Angus rating rating rating rating

$1234 \quad 1234 \quad 1234 \quad 1234$

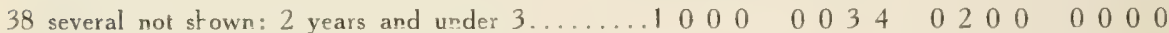

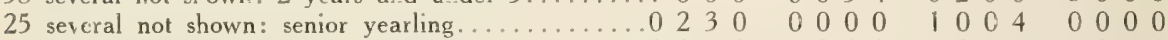

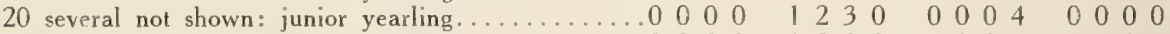

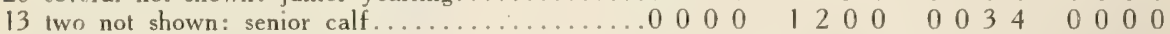

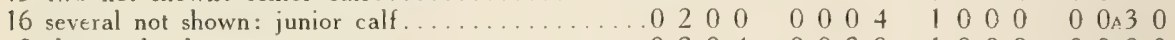

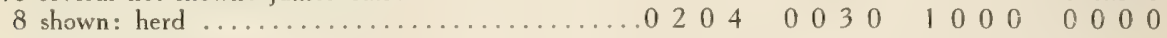

A Breed unknown.

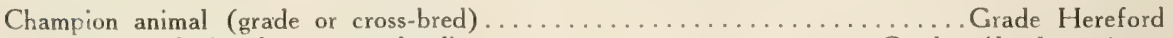

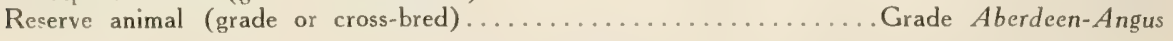

Champions by Ages (Pure-breds, Grades and Cross-breds Competing).

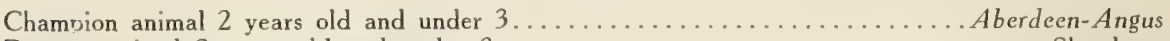
Reserve animal 2 years old and under $3 \ldots \ldots \ldots \ldots \ldots \ldots \ldots \ldots \ldots \ldots \ldots \ldots$ Shorthorn Champion animal 1 year old and under $2 \ldots \ldots \ldots \ldots \ldots \ldots \ldots \ldots \ldots \ldots \ldots$. . . . . . . . . . . . . . Reserve animal 1 year old and under $2 \ldots \ldots \ldots \ldots \ldots \ldots \ldots \ldots \ldots \ldots \ldots$ Aberden-Angus

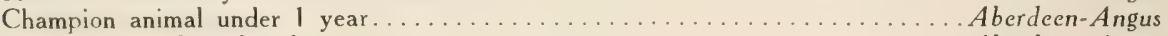

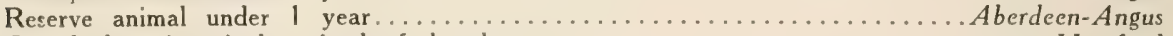

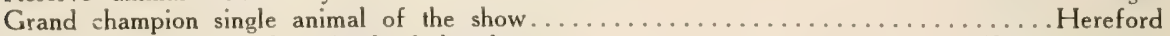
Reserve champion single animal of the show............................... Angus 
TOTAL ENTRIES.

CLASS.

Aberdeen-

Angus rating

Shorthorn rating

12345

$\begin{array}{lllll}0 & 0 & 3 & 0 & 0\end{array}$

$\begin{array}{lllll}0 & 0 & 0 & 0 & 0\end{array}$

$\begin{array}{lllll}0 & 0 & 0 & 0 & 0\end{array}$

$\begin{array}{lllll}0 & 0 & 3 & 0 & 0\end{array}$

$\begin{array}{lllll}0 & 0 & 3 & 0 & 0\end{array}$

() 00000
Hereford rating

rating

$12345 \quad 12345$

0200000000000

$\begin{array}{llllllllll}0 & 0 & 3 & 0 & 5 & 0 & 0 & 0 & 0 & 0\end{array}$

$\begin{array}{llllllllll}0 & 0 & 3 & 4 & 0 & 0 & 0 & 0 & 0 & 0\end{array}$

$12045 \quad 000000$

1000400000000

() 233000000000

Champion animal (grade or cross-bred)

Grade Aberdeen-Angus Reserve animal (grade or cross-bred).

Grade Aberdeen-Angus

\section{Champions by Ages (Pure-breds, Grades and Cross-breds Competing).}

Charrpion animal 2 years old and under 3.

Reserve animal 2 years old and under 3 ...

Grade Aberdeen-Angus Champion animal 1 year old and under 2

Reserve animal 1 year old and under 2 . Shorthorn

Champion animal under 1 year. .

Reserve animal under 1 year

Champion herd of the show.

Reserve herd of the show.

Grand champion single animal of the show.

Reserve champion single animal of the show Hereford Aberdeen-Angus Aberdeen-Angus Hereford

Grade Aberdeen-Angus Abericen-Angus Grade Aberdeen-Angus Hereford

1903

TGTAL ENTRIES.

CLASS.

Aberdeen-

Angus raling

Shorthorn

Hereford

Mixed

12345

34 several not shown: 2 years and under 3..0 20005

19 several not shown: senior yearling...... 100000

20 several not shown: junior yearling. ...... 0232300

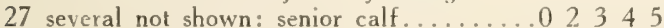

12 several not shown: junior calf........ 0003003

9 shown: herd ...................... 23340

15 several not shown: get of one sire.....00300 *Shorthorn-Galloway. zGalloway.

rating

\section{$123+5$}

raling

raling

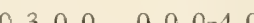

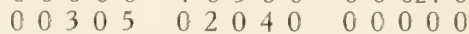

$00000 \quad 10045 \quad 000000$

$\begin{array}{lllllllllllllll}0 & 0 & 0 & 0 & 0 & 1 & 0 & 0 & 0 & 0 & 0 & 0 & 0 & 0 & 0\end{array}$

$\begin{array}{llllllllllllllllll}0 & 0 & 0 & 4 & 0 & 0 & 0 & 0 & 0 & 0 & * & * & * & 0 & 0 & 0\end{array}$

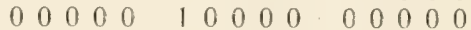

$00000 \quad 12000000000$

Champion animal (grade or cross-bred). Hereford Reserve animal (grade or cross-bred). Hereford

\section{Champions by Ages (Pure-breds, Grades and Cross-breds Competing),}

Champion animal 2 years old and under $3 \ldots \ldots \ldots \ldots \ldots \ldots \ldots \ldots \ldots \ldots \ldots \ldots \ldots \ldots$. Reserve animal 2 years old and under $3 \ldots \ldots \ldots \ldots \ldots \ldots \ldots \ldots \ldots \ldots \ldots$. . . . . . . . . . . . . . . Champion animal 1 year old and under $2 \ldots \ldots \ldots \ldots \ldots \ldots \ldots \ldots \ldots$ Aberdeen-Angus Reserve animal 1 year old and under $2 \ldots \ldots \ldots \ldots \ldots \ldots \ldots \ldots \ldots \ldots \ldots \ldots \ldots \ldots \ldots$.

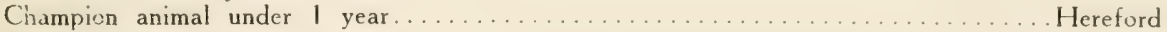

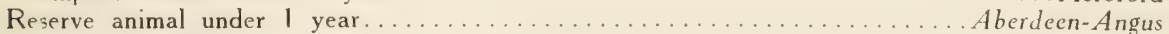

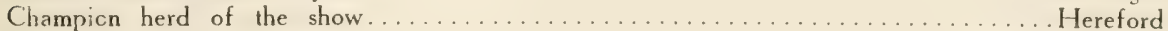

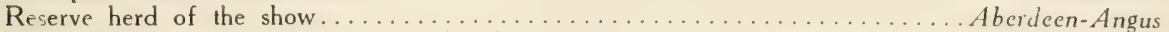

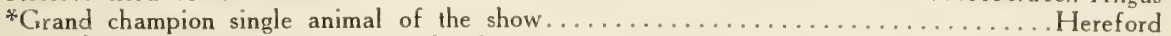

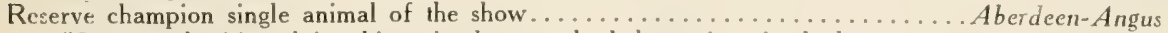
*Some authorities claim this animal correctly belongs in mixed class. 
IOTAL ENTRIES.

Aberdeen-

Angus rating

Shorthorn

rating

12345

12345

32 several not shown: 2 years and under 3..00000 0

23 several not shown: senior yearling......1 03340

21 several not shown: junior yearling......0 00000

20 several not shown: senior calf........02230 5

10 several not shown: junior calf........ 000000

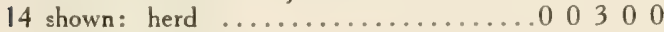

II shown: get of one sire.............. 2000

Hereford

Mixed

rating

rating

10040.0233000000000

$\begin{array}{lllllllllllllll}0 & 0 & 0 & 0 & 0 & 0 & 2 & 0 & 0 & 5 & 0 & 0 & 0 & 0 & 0\end{array}$

(1) $\begin{array}{lllllllllllllllllll}0 & 0 & 0 & 0 & 0 & 0 & 3 & 4 & 5 & * & 1 & 2 & 0 & 0 & 0\end{array}$

$\begin{array}{lllllllllllllllll}0 & 0 & 0 & 0 & 0 & 1 & 0 & 0 & 0 & 0 & 0 & 0 & 0 z & 0 & 0\end{array}$

$\begin{array}{llllllllllll}0 & 0 & 0 & 4 & 0 & 0 & 0 & 3 & 0 & 5 & * & * \\ 2 & 0 & 0 & 0\end{array}$

$\begin{array}{llllllllllllllll}0 & 0 & 0 & 0 & 0 & 1 & 0 & 0 & 4 & 0 & 0 b 2 & 0 & 0 & 0\end{array}$

$\begin{array}{llllllllllllllll}0 & 0 & 0 & 0 & 0 & 0 & 0 & 3 & 0 & 0 & \mathrm{x} 1 & 0 & 0 & 0 & 0\end{array}$

*Shorthorn-Galloway. bMixed herd. xMixed or cross-bred. zGalloway.

Champion animal (grade or cross-bred)

Grade Shorthorn

Reserve animal (grade or cross-bred).

Shorthoin-Galloway

Champions by ages (Plre-breds, Craces and Cross-breds Competing).

Champion animal 2 years old and under 3

Aberdeen-Angus

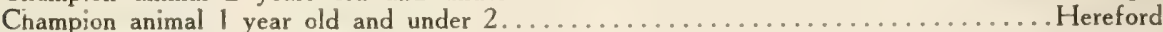

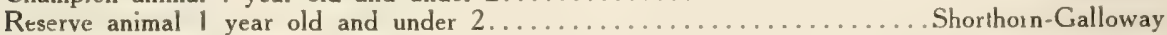

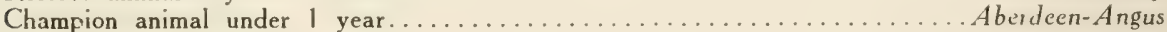

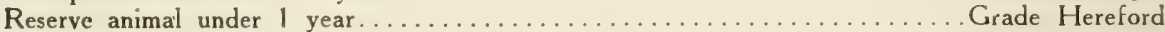

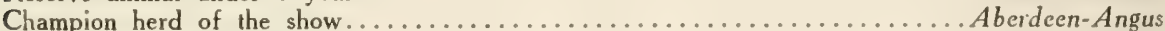

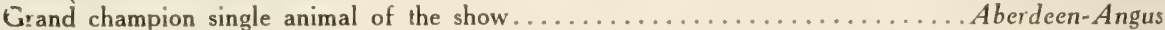

Reserve champion single animal of the show

Hereford

1905

$\begin{array}{lcccc}\text { Aberdeen- } & \begin{array}{c}\text { Shorthorn } \\ \text { rating }\end{array} & \begin{array}{c}\text { Hereford } \\ \text { rating }\end{array} & \begin{array}{c}\text { Mixed } \\ \text { raling }\end{array}\end{array}$

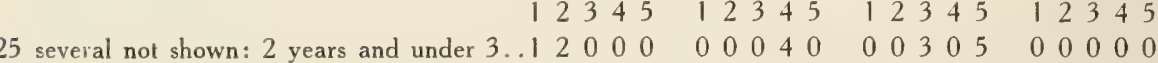

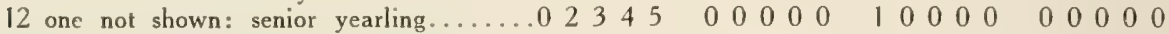

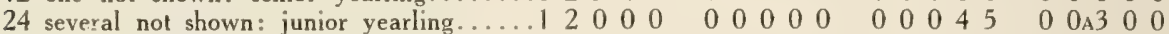

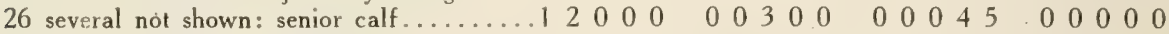

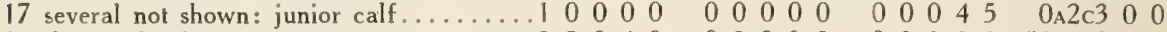

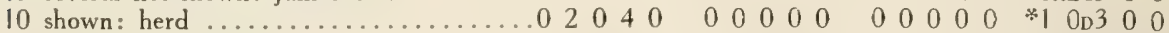

9 shown: get of one sire........... 2330000000000000000000000

* Aberdeen-Angus-Shorthorn-Galloway. cAberdeen-Angus-Hereford. DBreed unknown.

AShorthorn-Galloway.

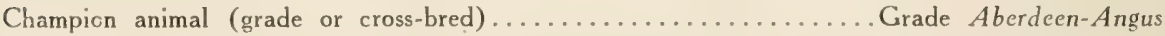

\section{Champions by Ages (Pure-breds, Grades and Cross-breds Competing).}

Champion animal 2 years old and under $3 \ldots \ldots \ldots \ldots \ldots$ Grade Aberdeen-Angus

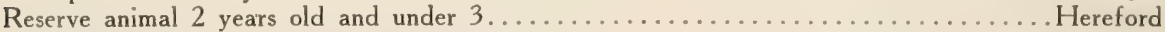

Champion animal 1 year old and under $2 \ldots \ldots \ldots \ldots \ldots \ldots \ldots \ldots \ldots \ldots \ldots$. $\ldots \ldots \ldots$ ford

Reserve animal $\mid$ year old and under $2 \ldots \ldots \ldots \ldots \ldots \ldots \ldots \ldots \ldots \ldots$ Aben-Angus

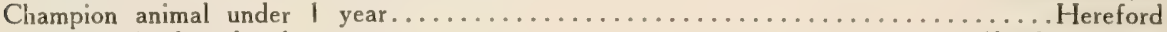

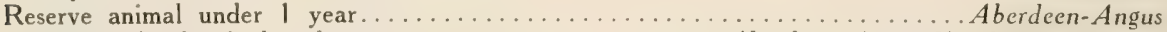

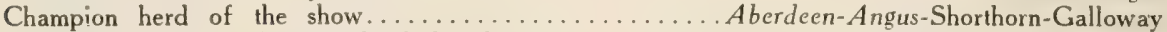

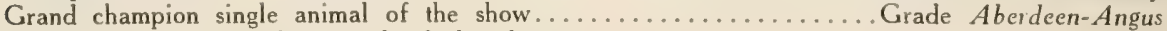

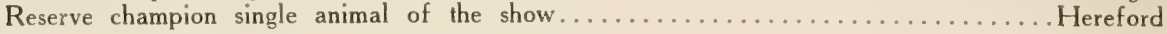


TOTAL ENTRIES.

CLASS.

AberdeenAngus rating

Shorthorn rating

12345

12345

28 two not shown: 2 years and under $3 \ldots 10045$

21 several not shown: senior yearling.....0 00300

22 several not shown: junior yearling....... 00000

16 two not shown: senior calf............ 2305

13 several not shown: junior calf........ 1004040

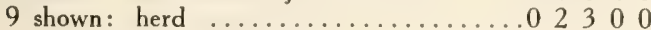

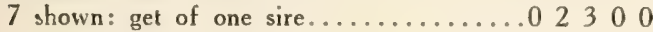
BShorthorn-Calloway. $\begin{array}{lllllllllllllll}0 & 2 & 0 & 0 & 0 & 0 & 0 & 0 & 0 & 0 & 0 & 0 \mathrm{~A} & 0 & 0 & 0\end{array}$

$020000000045 * 100000$

$\begin{array}{llllllllllllll}0 & 0 & 3 & 0 & 0 & 0 & 2 & 0 & 0 & 5 & 0 & 0 & 0_{\mathrm{B}} 4 & 0\end{array}$

$\begin{array}{lllllllllllllll}0 & 0 & 0 & 0 & 0 & 0 & 0 & 0 & 4 & 0 & 0 & 0 & 0 & 0 & 0\end{array}$

$\begin{array}{llllllllllllll}0 & 0 & 0 & 0 & 0 & 0 & 2 & 0 & 0 & 0 & 0 & 0 \mathrm{c} 3 & 0 * 5\end{array}$

$\begin{array}{llllllllllllll}0 & 0 & 0 & 0 & 0 & 1 & 0 & 0 & 0 & 0 & 0 & 0 & 0 \mathrm{D} 4 & 0\end{array}$

$\begin{array}{llllllllllllll}0 & 0 & 0 & 0 & 1 & 0 & 0 & 0 & 0 & 0 & 0 & 0 & 0 & 0\end{array}$

*Hereford-Aberdeen-Angus.

Champion animal (grade or cross-bred)

Hereford-Aberdeen-Angus

\section{Champions by Ages (Pure-breds, Grades and Cross-breds Competing).}

Champion animal 2 years old and under 3

Champion herd of the show

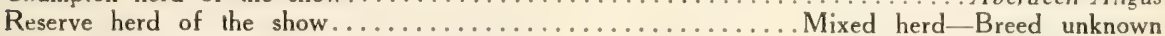

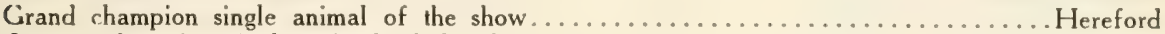

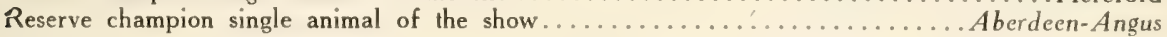

\begin{tabular}{|c|c|c|c|c|}
\hline ENTRIES. & $\begin{array}{l}\text { Aberdeen- } \\
\text { Angus rating }\end{array}$ & $\begin{array}{l}\text { Shorthorn } \\
\text { rating }\end{array}$ & $\begin{array}{l}\text { Hereford } \\
\text { rating }\end{array}$ & $\begin{array}{l}\text { Mixed } \\
\text { raling }\end{array}$ \\
\hline & 12345 & 12345 & 12345 & 12345 \\
\hline th 2 & 0034 & $\begin{array}{lllll}0 & 0 & 0 & 0 & 0\end{array}$ & 02005 & $* 100$ \\
\hline & 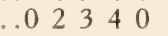 & $\begin{array}{lllll}0 & 0 & 0 & 0 & 5\end{array}$ & 10000 & 0000 \\
\hline & 00300 & $\begin{array}{lllll}0 & 0 & 0 & 0 & 0\end{array}$ & 12040 & $\begin{array}{llll}0 & 0 & 0 & 0 \mathrm{~A} 5\end{array}$ \\
\hline & .02300 & 10005 & 0004 & 0000 \\
\hline & .12000 & $\begin{array}{lllll}0 & 0 & 0 & 0 & 0\end{array}$ & 00034 & 000 \\
\hline & & $\begin{array}{lllll}0 & 0 & 0 & 0\end{array}$ & 100000 & 000 \\
\hline bo & 12300 & $\begin{array}{lllll}0 & 0 & 0 & 0 & 0\end{array}$ & $\begin{array}{lllll}0 & 0 & 0 & 0 & 0\end{array}$ & 000 \\
\hline
\end{tabular}

* Aberdeen-Angus-Shorthorn. AAberdeen-Angus-Hereford.

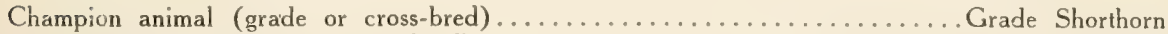

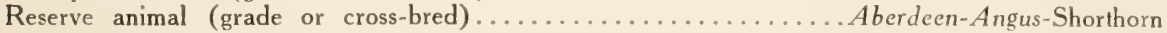

\section{Champions by Ages (Pure-breds, Grades and Cross-breds Competing).}

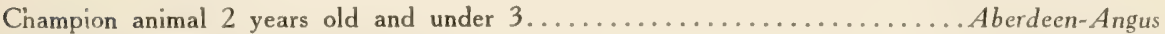

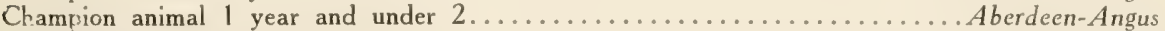

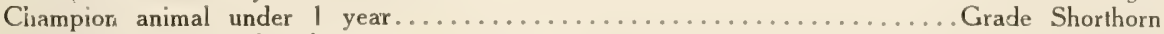

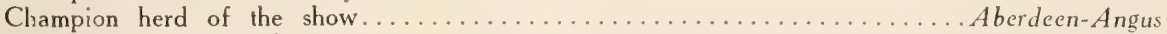

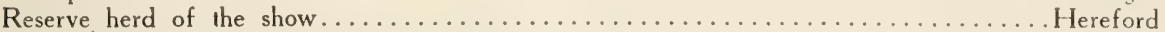

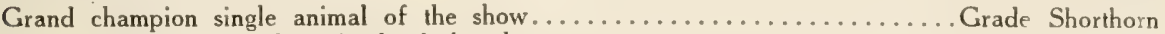
Reserve champion single animal of the show............................. Angus 
TOTAL ENTRIES.

Aberdeen-

Angus raling

12345

Shorthorn rating

12345

$\begin{array}{lllll}0 & 0 & 0 & 0 & 5\end{array}$

10000

$\begin{array}{lllll}0 & 0 & 0 & 4 & 0\end{array}$

I 2340

$\begin{array}{lllll}0 & 0 & 0 & 0 & 5\end{array}$

02040

10000
Hereford
rating

$123+5$

10040

$\begin{array}{lllll}0 & 0 & 3 & 0 & 0\end{array}$

$\begin{array}{lllll}0 & 0 & 0 & 0 & 0\end{array}$

$\begin{array}{lllll}0 & 0 & 0 & 0 & 0\end{array}$

$\begin{array}{lllll}0 & 0 & 3 & 4 & 0\end{array}$

$\begin{array}{lllll}0 & 0 & 0 & 0 & 0\end{array}$

00300
Mixed

rating

12345

00000

$\begin{array}{lllll}0 & 0 & 0 & 0 & 0\end{array}$

$\begin{array}{llllll}0 & 0 & 0 & 0 & 0\end{array}$

$\begin{array}{llllllll}0 & 0 & 0 & 0 & 0\end{array}$

$\begin{array}{lllllllllll}0 & 0 & 0 & 0 & 0\end{array}$

$\begin{array}{lllllllllll}0 & 0 & 0 & 0 & 0\end{array}$

$\begin{array}{lllllllllll}0 & 0 & 0 & 0 & 0\end{array}$

Champion animal (grade or cross-bred)

Grade Shorthorn

Reserve animal (grade or cross-bred).

Grade Hereford

\section{Champions by Ages (Pure-breds, Grades and Cross-breds Competing).}

Champion animal 2 years old and under 3.

Reserve animal 2 years old and under 3.

Champion animal 1 year old and under 2

Abericen-Angus Aberdecn-Angus

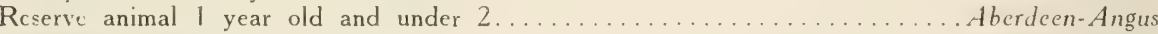

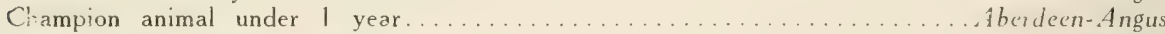

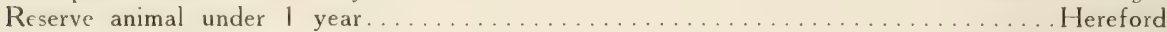

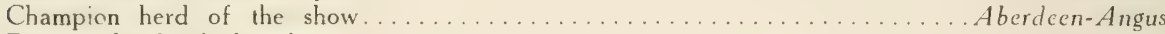

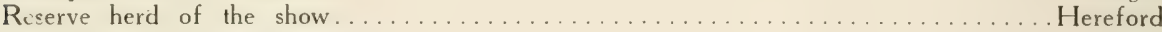

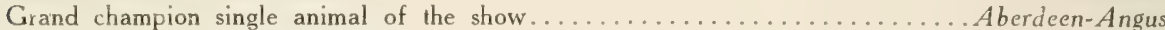

Reserve champion single animal of the show..................... Grade Shorthorn

\section{9}

TOTAL ENTRIES.

CLASS

\section{2 years and under 3}

12 Senior yearling

31 Juninx yearling

20 Senior calf

12 Juinior calf

9 Herd

$17 \mathrm{Get}$ of one sire.

10340

$\begin{array}{lllll}1 & 0 & 3 & 4 & 5\end{array}$

$\begin{array}{cccc}\text { Aberdeen- } & \begin{array}{c}\text { Shorthorn } \\ \text { raling }\end{array} & \begin{array}{c}\text { Hereford } \\ \text { raing }\end{array} & \begin{array}{c}\text { Mixed } \\ \text { rating }\end{array}\end{array}$

12345

12345

12345

12345

020005

00305

020000

$\begin{array}{llllll}0 & 0 & 0 & 0 & 0\end{array}$

100000

$\begin{array}{lllllllllll}0 & 0 & 0 & 0 & 0\end{array}$

$\begin{array}{llllll}0 & 0 & 0 & 0 & 0\end{array}$

$\begin{array}{llllll}0 & 0 & 0 & 0 & 0\end{array}$

$\begin{array}{llllll}0 & 0 & 0 & 0 & 0\end{array}$

C. 2005

$\begin{array}{lllll}0 & 0 & 0 & 0 & 0\end{array}$

$\begin{array}{lllll}0 & 0 & 0 & 0 & 5\end{array}$

$\begin{array}{lllll}0 & 0 & 0 & 0 & 0\end{array}$

10000

$\because 10.300$

$\begin{array}{lllll}0 & 0 & 0 & 0 & 0\end{array}$

$\begin{array}{lllll}0 \mathrm{~B} & 0 \mathrm{c} 4 & 0\end{array}$

$\begin{array}{llllll}0 & 0 & 0 & 0 & 0\end{array}$

$\begin{array}{lllllllllll}0 & 0 & 0 & 0 & 0\end{array}$

$\begin{array}{llllllllll}0 & 0 & 0 & 0 & 0\end{array}$

0030

BMixed-1 Shortho:n and 2 AberdeenAngus.. cMixed-1 grade Aberdeen-Angus, 1 grade Hereford, 1 cross-bred Hereford-AberdeenAngus.

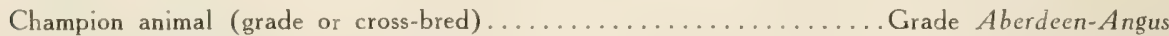
Reserve animal (grade or cross-bred) ................................. Hereford

\section{Champions by Ages (Pure-breds, Grades and Cross-breds Competing).}

Champion animal 2 years old and under $3 \ldots \ldots \ldots \ldots \ldots \ldots \ldots$ Aberdeen-Angus Reserve animal 2 years old and under $3 \ldots \ldots \ldots \ldots \ldots \ldots \ldots \ldots$ Aberdeen-Angus

Champion animal $\mid$ year old and under $2 \ldots \ldots \ldots \ldots \ldots \ldots \ldots \ldots \ldots$ Aberdeen-Angus

Reserve animal 1 year old and under $2 \ldots \ldots \ldots \ldots \ldots \ldots \ldots \ldots \ldots \ldots \ldots \ldots \ldots \ldots \ldots$ Aben-Angus

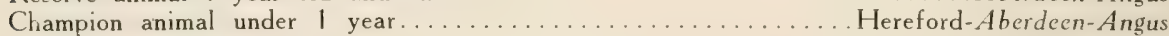

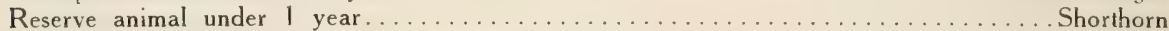

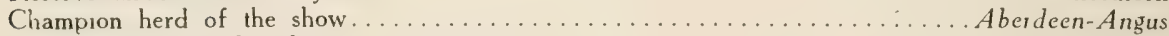

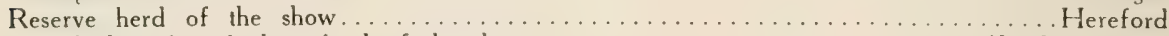

Grand champion single animal of the show.................................

Reserve champion single animal of the show...................... Aberdeen-Angus 
TOTAL ENTRIES.

CLASS.

Aberdeen-

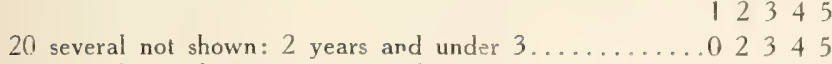

Angus rating
12345

Shorihorn

Hereford

12345

rating rating

$123+5123+5$

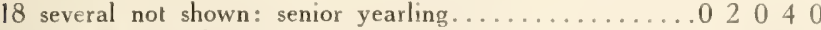

$\begin{array}{lllllllllll}0 & 0 & 0 & 0 & 0 & 1 & 0 & 0 & 0 & 0\end{array}$

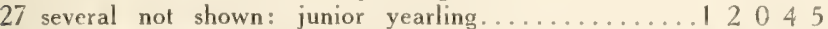

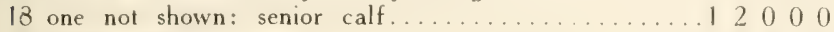

$\begin{array}{llllllllll}0 & 0 & 0 & 0 & 0 & 1 & 0 & 3 & 0 & 5\end{array}$

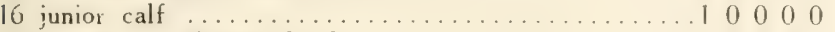

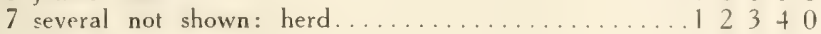

10 Get of one sire.

.02300

$\begin{array}{llllllllll}0 & 0 & 3 & 0 & 0 & 0 & 0 & 0 & 0 & 0\end{array}$

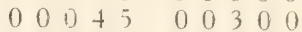

$\begin{array}{llllllllll}0 & 2 & 0 & 0 & 5 & 0 & 0 & 3 & 4 & 0\end{array}$

$\begin{array}{lllllllllll}0 & 0 & 0 & 0 & 0 & 0 & 0 & 0 & 0 & 0\end{array}$

$\begin{array}{llllllllll}1 & 6 & 0 & 0 & 0 & 0 & 0 & 0 & 0 & 0\end{array}$

Champion animal (grade or cross-bred).

Grade Aberdeen-Angus Reserve champion animal (grade or cross-bred)........... Grade Hereford-Aberdeen-Angus

\section{Champions by Ages (Pure-breds, Grades and Cross-breds Competing).}

Champion animal 2 years old and under $3 \ldots \ldots \ldots \ldots \ldots$ Shorthorn

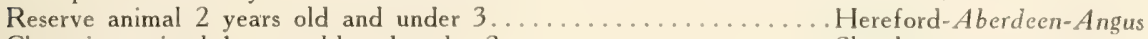

Champion animal 1 year old and under $2 \ldots \ldots \ldots \ldots$ Shorthorn

Reserve animal I year and under $2 \ldots \ldots \ldots \ldots \ldots \ldots \ldots$ Aberdeen-Angus

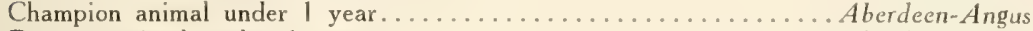

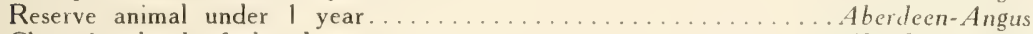

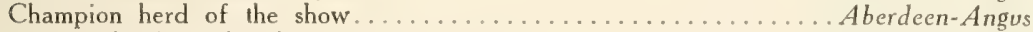

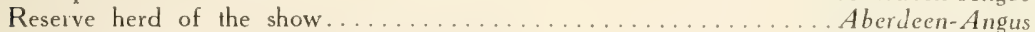

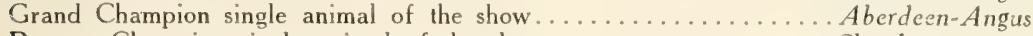

Reserve Champion single animal of the show.............. Shorthorn

1911

\begin{tabular}{|c|c|c|c|c|}
\hline L. ENTRIES. & CLASS. & $\begin{array}{l}\text { Aberdeen- } \\
\text { Angus rating }\end{array}$ & $\begin{array}{c}\text { Shorthorn } \\
\text { rating }\end{array}$ & $\begin{array}{l}\text { Hereford } \\
\text { rating }\end{array}$ \\
\hline & & 12345 & $123+5$ & 1234 \\
\hline $\operatorname{ar}$ & & .12345 & $\begin{array}{lllll}0 & 0 & 0 & 0 & 0\end{array}$ & $\begin{array}{llll}0 & 0 & 0 & 0\end{array}$ \\
\hline yearl & & .12345 & $\begin{array}{lllll}0 & 0 & 0 & 0 & 0\end{array}$ & 0000 \\
\hline yearling & & .12340 & 00005 & 0000 \\
\hline$r$ calf .. & & .02340 & $\begin{array}{lllll}0 & 0 & 0 & 0 & 5\end{array}$ & 100 \\
\hline calf & & $123+0$ & $\begin{array}{lllll}0 & 0 & 0 & 0 & 0\end{array}$ & $\begin{array}{lll}0 & 0 & 0\end{array}$ \\
\hline & & .12340 & $\begin{array}{lllll}0 & 0 & 0 & 0 & 0\end{array}$ & $\begin{array}{lll}0 & 0 & 0\end{array}$ \\
\hline one & & .10300 & $\begin{array}{lllll}0 & 0 & 0 & 0 & 0\end{array}$ & 020 \\
\hline
\end{tabular}

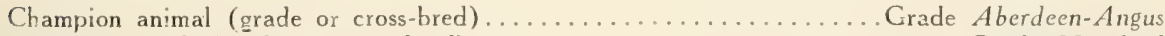

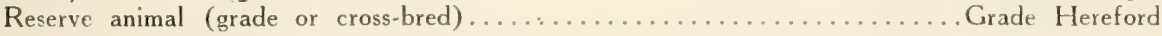

\section{Champions by Ages (Pure-breds, Grades and Cross-breds Competing).}

Champion animal 2 years old and under $3 \ldots \ldots \ldots \ldots \ldots$ Crade Aberdeen-Angus Reserve champion animal 2 years old and under $3 \ldots \ldots \ldots \ldots$. . . . . . Champion animal 1 year old and under $2 \ldots \ldots \ldots \ldots \ldots \ldots$. . . . . . . . . Aberdeen-Angus Reserve champion animal 1 year old and under $2 \ldots \ldots \ldots \ldots \ldots$. . . . . . . Aberdeen-Angus

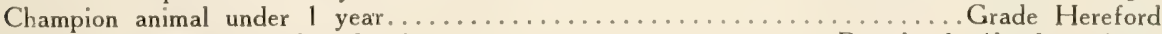
Reserve champion animal under 1 year.................... Pure-bred Aberdeen-Angus

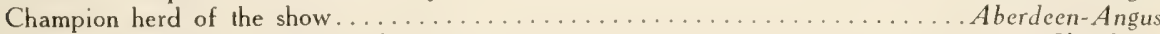
Reserve champion herd of the show . . . . . . . . . . . . . . . . . . . . . . . . . .

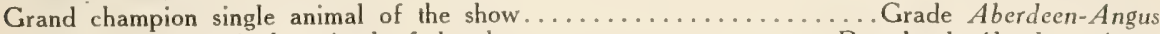
Reserve champion single animal of the show................ Pure-bred Aberdeen-Angus 


\begin{tabular}{|c|c|c|c|}
\hline TOIAL ENTRIES. CLASS. & $\begin{array}{l}\text { Abeideen- } \\
\text { Angus rating }\end{array}$ & $\begin{array}{l}\text { Shorthorn } \\
\text { rating }\end{array}$ & $\begin{array}{l}\text { Hereford } \\
\text { rating }\end{array}$ \\
\hline wo years and irds 3. & .10045 & $\begin{array}{lllll}0 & 0 & 3 & 0 & 0\end{array}$ & 02000 \\
\hline or yearling & .1000005 & 02040 & $\begin{array}{lllllllllllllllll}0 & 0 & 3 & 0 & 0\end{array}$ \\
\hline ior yearling & .12345 & $\begin{array}{llllll}0 & 0 & 0 & 0 & 0\end{array}$ & 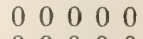 \\
\hline iior calf & .1000005 & 022340 & $\begin{array}{llllll}0 & 0 & 0 & 0 & 0\end{array}$ \\
\hline $\begin{array}{l}\text { lor calt } \\
\text { rd } \ldots \ldots\end{array}$ & $\begin{array}{lllll}1 & 0 & 0 & 4 & 5 \\
1 & 0 & 0 & 0 & 0\end{array}$ & $\begin{array}{lllllll}0 & 0 & 0 & 0 & 0 \\
0 & 0 & 3 & 0 & 0\end{array}$ & $\begin{array}{llllllll}0 & 2 & 3 & 0 & 0 \\
0 & 2 & 0 & 4 & 0\end{array}$ \\
\hline of one sire. & .10300 & $\begin{array}{llllll}0 & 0 & 0 & 0 & 0\end{array}$ & $\begin{array}{lllll}0 & 2 & 0 & 4 & 0 \\
0 & 2 & 0 & 0 & 0\end{array}$ \\
\hline
\end{tabular}

Crampion animal (grade or cross-bred)

Reserve champion animal (grade or cross-bred)

Grade Aberdecn-Angus Grade Aberdeen-Angus

\section{Champions by Ages (Pure-breds, Grades and Cross-breds Competing).}

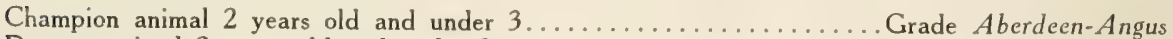
Reserve animal 2 years old and under $3 \ldots \ldots \ldots \ldots \ldots \ldots \ldots \ldots \ldots \ldots \ldots \ldots \ldots$. Champion animal 1 year old and under $2 \ldots \ldots \ldots \ldots \ldots \ldots \ldots \ldots$ Grade Aberdeen-Angus Reserve animal I year and under $2 \ldots \ldots \ldots \ldots \ldots \ldots \ldots \ldots \ldots$. Pure-bred Shorthorn

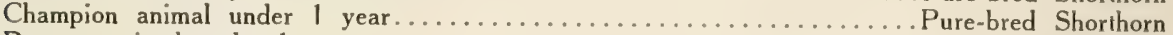

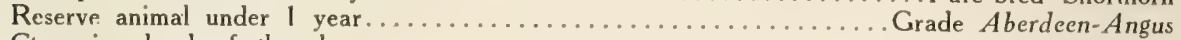
Champion herd of the show......................................... Angus

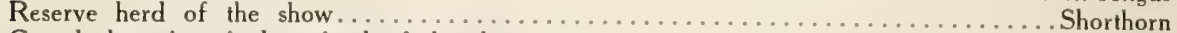

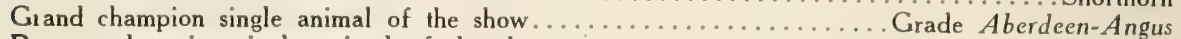

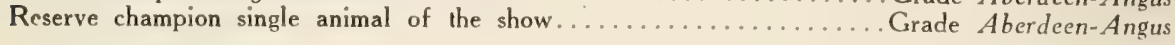

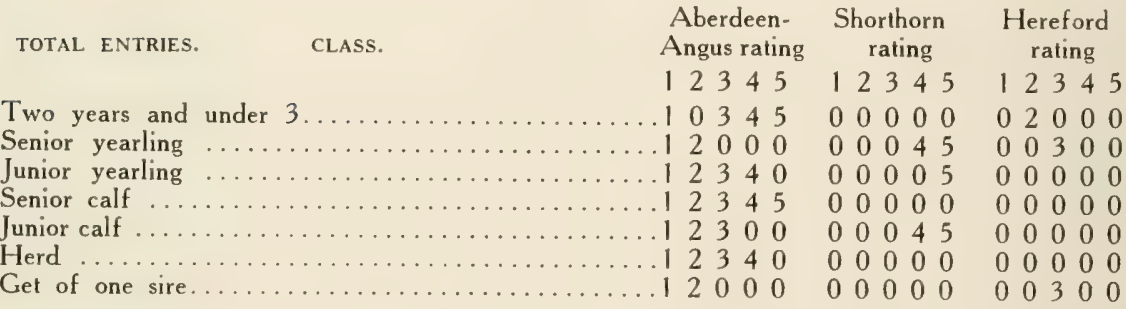

Champion animal (grade or cross-bred.

Grade Aberdeen-Angus

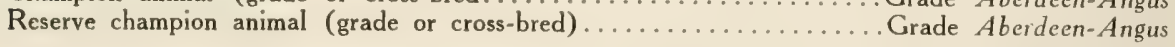

\section{Champions by Ages (Pure-breds, Grades and Cross-breds Competing).}

Champion animal 2 years old and under $3 \ldots \ldots \ldots \ldots \ldots$. Grade Aberdeen-Angus Reserve animal 2 years old and under $3 \ldots \ldots \ldots \ldots \ldots \ldots \ldots$. Pure-bred Shorthorn Champion animal I year old and under $2 \ldots \ldots \ldots \ldots \ldots \ldots \ldots$ Grade Aberdeen-Angus Reserve animal I year and under $2 \ldots \ldots \ldots \ldots \ldots \ldots \ldots \ldots \ldots$. Pure-bred Hereford Champion animal under 1 year................................ Hured Hereford

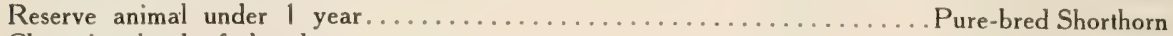

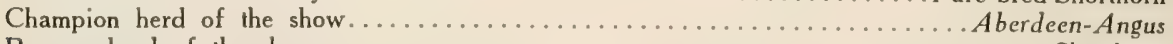

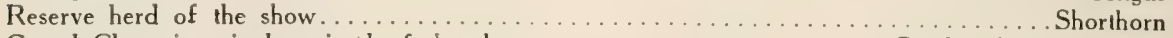
Grand Champion single animal of the show.................. Grade Aberdeen-Angus Reserve Champion single animal of the show................. Grade Aberdeen-Angus 


\title{
Summary of Single Steer, Spayed or Martin Heifer Competition
}

\author{
GRAND CHAMPION SINGLE STEER AWARDS.
}

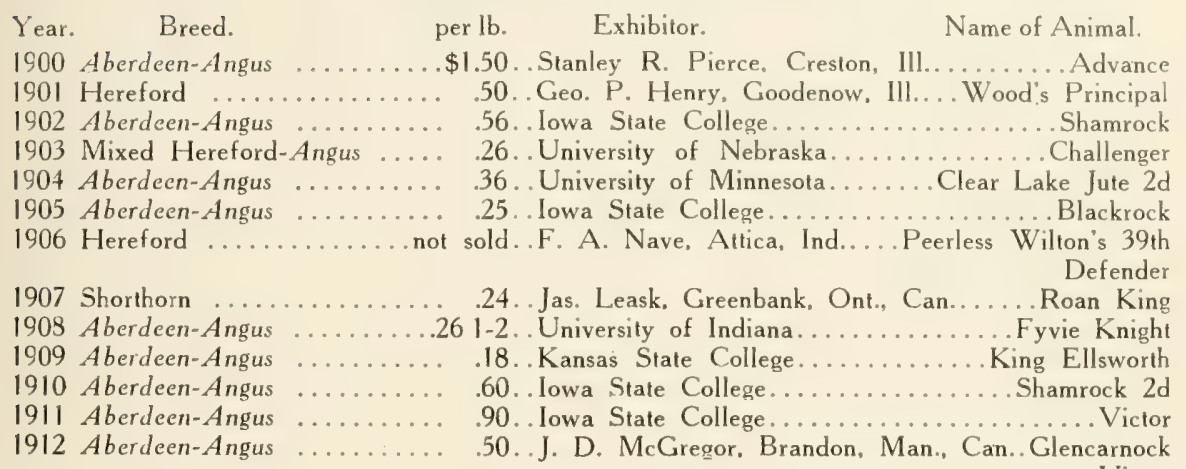

1913 Aberdeen-Angus ........ not sold. J. D. McGregor, Brandon, Man., Can.. Glencarnock Victor $2 \mathrm{~d}$

A complete analysis of a tabulated list of awards of the single steer or heifer competition reveals very forcibly the substantial steady growth the Aberdeen-Angus breed has made since the inception of the International Live Stock Exposition. A careful review of the awards in the classes in which single animals of all breeds competed should prove very interesting to admirers of Aberdeen-Angus because it shows very clearly that while the Hereford and Shorthorn breeds were conspicuous by their winnings the first few years of the show, it has become apparent during recent years that the Aberdeen-Angus bullocks have gained prizes in overwhelming numbers over the Hereford and Shorthorn.

The actual proof of the superiority of the Aberdeen-Angus breed is set forth in the following brief synopsis of comparisons which covers fourteen International Live Stock Expositions.

In competing for Championships, out of fourteen possible Championships for Grades and Cross-breds, Aberdeen-Angus won eight. Herefords iwo, Shorthorns three, mixed HerefordAterdeen-Angus one, and in the same classes only twelve Reserves were reported, which were won as follows: Aberdeen-Angus four, Herefords five, one mixed Shorthorn-Galloway, one mixed Aberdeen-Angus-Shorthorn, and one mixed Hereford-Aberdeen-Angus.

Out of fourteen possible Granci Championships and fourteen Reserves (competition open to pure-breds, grades and cross-breds) Aberdeen-Angus won ten Grand Championships and nine Reserve Grand Championships, Herefords won three of each, and the Shorthorns one of each.

Ou: of twelve Grand Champion steer herd prizes, Aberdeen-Angus won ten, Herefords one. and a mixed herd of Aberdeen-Angus-Shorthorn-Galloway one. Only ten of the twelve Reserve Grand Champion steer herd prizes were reported, four being won by Aberdeen-Angus, three by Herefords, two by Shorthorns, and one by a mixed herd.

Out of forty-two possible Champion awards by ages (pure-breds, grades ond cross-breds competing), Aberdeen-Angus won twenty-four, Herefords eleven, Shorthorns six, mixed Hereford-Aberdeen-Angus one, and out of thirty-eight Reserves which were reported, Aberdeen-Angus won twenty, Herefords nine, Shorthorns seven, mixed Shorthorn-Galloway one, Hereford-Aberdeen-Angus one.

Out of the grand total of one hundred and fifty-seven Champion and Reserve Champion avards. Aberdeen-Angus won ninety, Herefords thirty-seven, Shorthorns twenty-one, and mixed nine.

These facts should serve as conclusive proof of the superior merits of the Aberdeen-Angus breed in the single steer and heifer competition. 


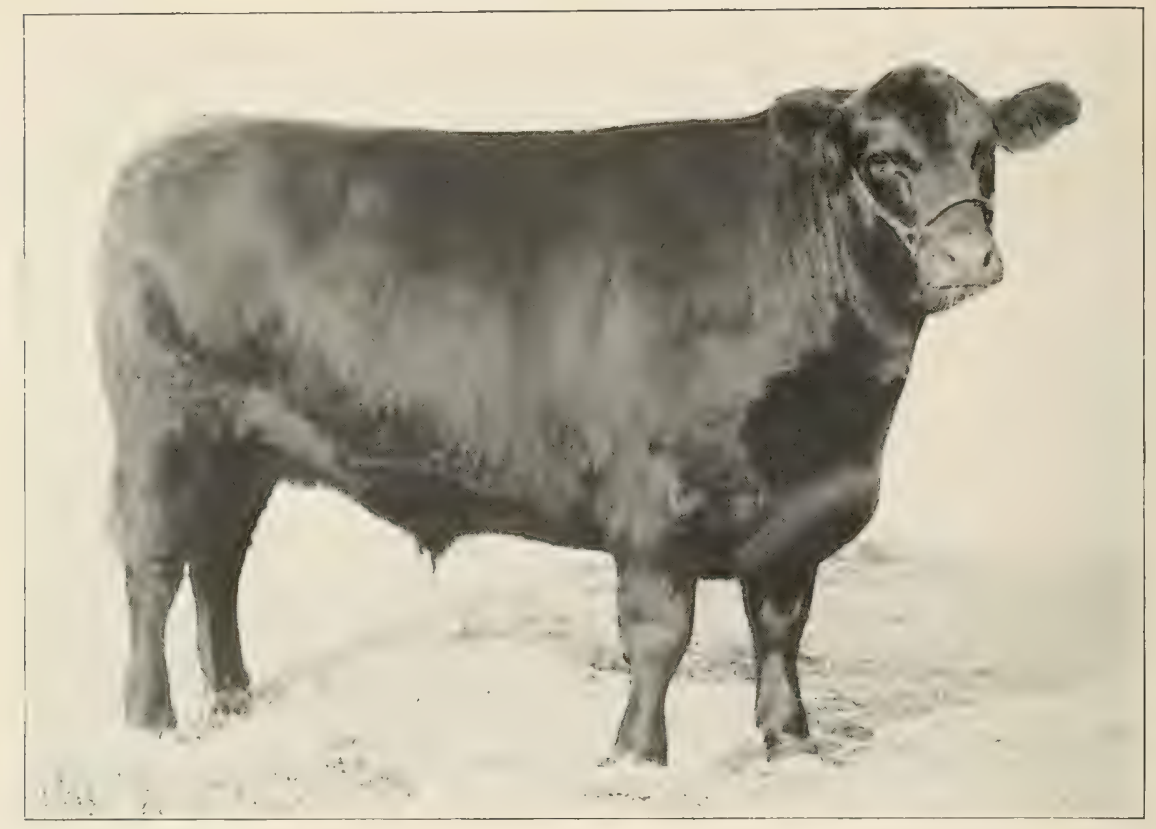

KING ELLSWORTH

An Aberdeen-Angus Product of Kansas.

1990 International Live Stock Exposition Grand Champion Steer over all Breeds. Fed and Exhibited by Kansas State College.

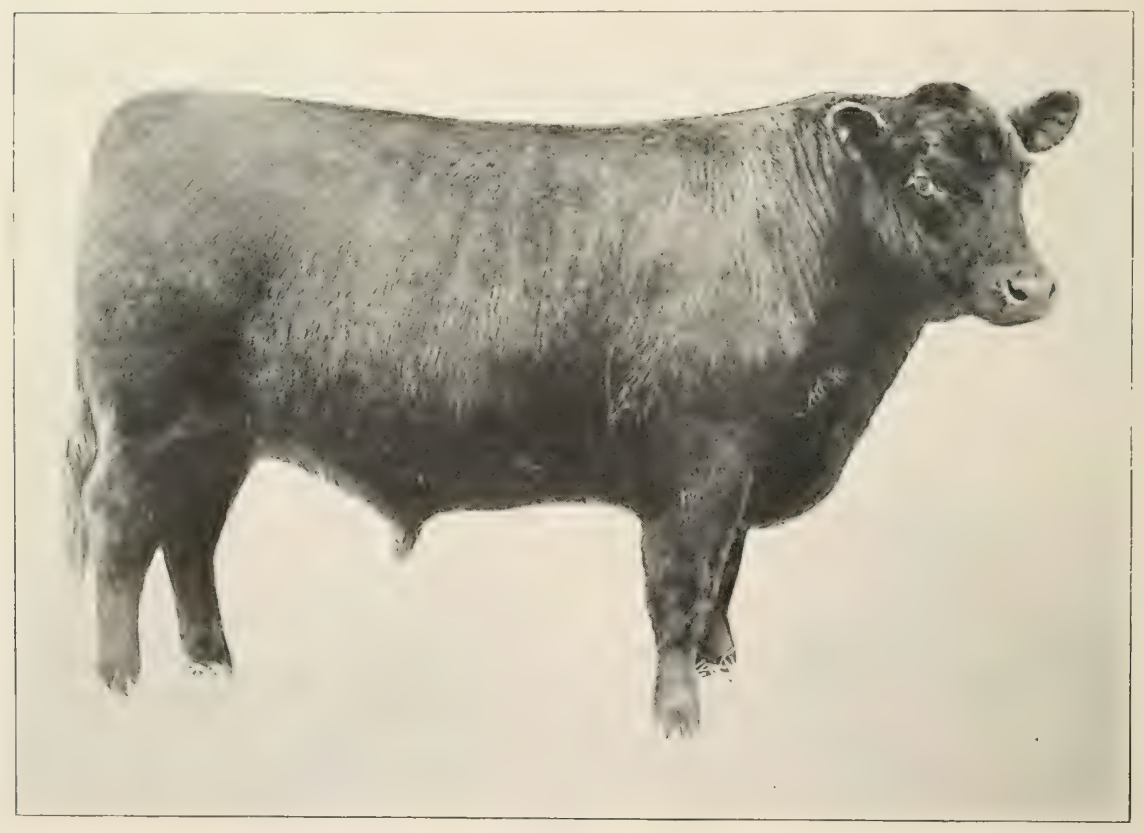

SHAMROCK II.

An Aberdeen-Angus Product of Iowa.

1910 International Live Stock Exposition Grand Champion Steer over all Breeds. Ferl and Exhibited by Iowa State College, Ames. Inwa. 


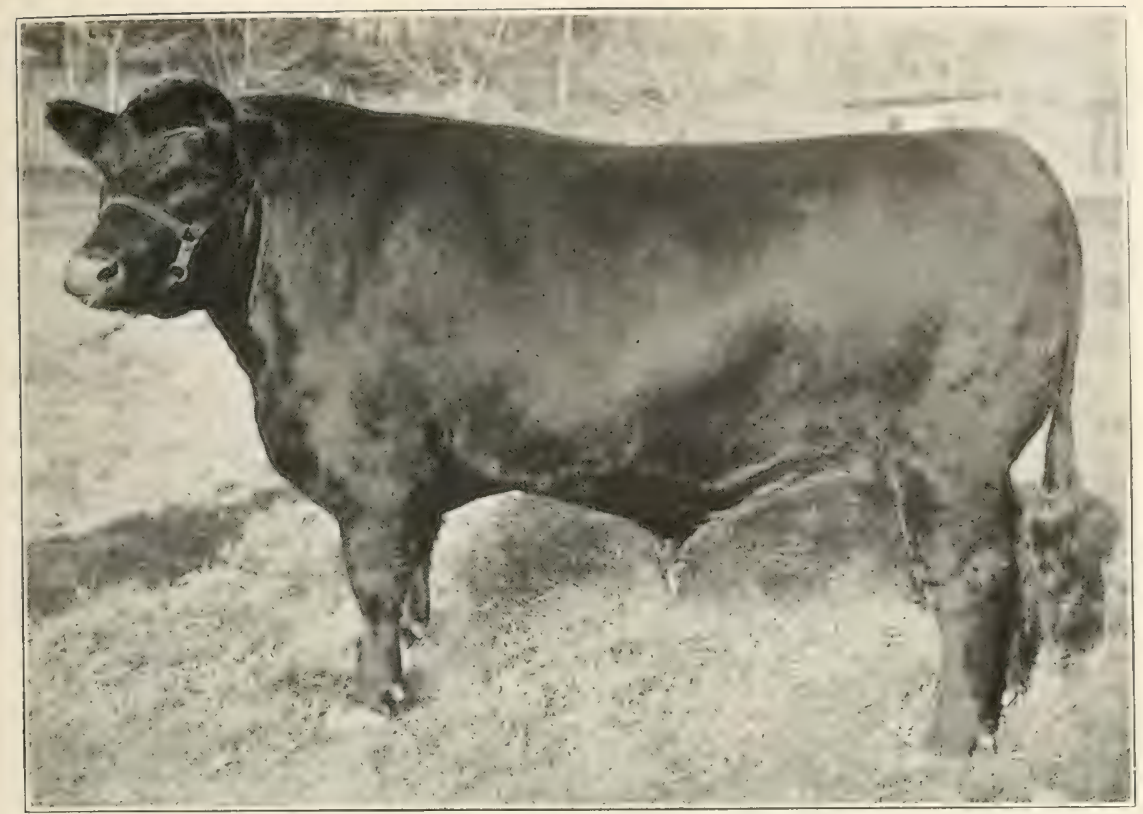

CI,EAR I,AKE JUTE 20

An Aberdeen-Angus P'roduct of Minnesota

1:04 International Live Stock Exposition Grand ('hampion Steer over all Breeds. Fetl and Exhibited by Lniversity of dlimnesota.

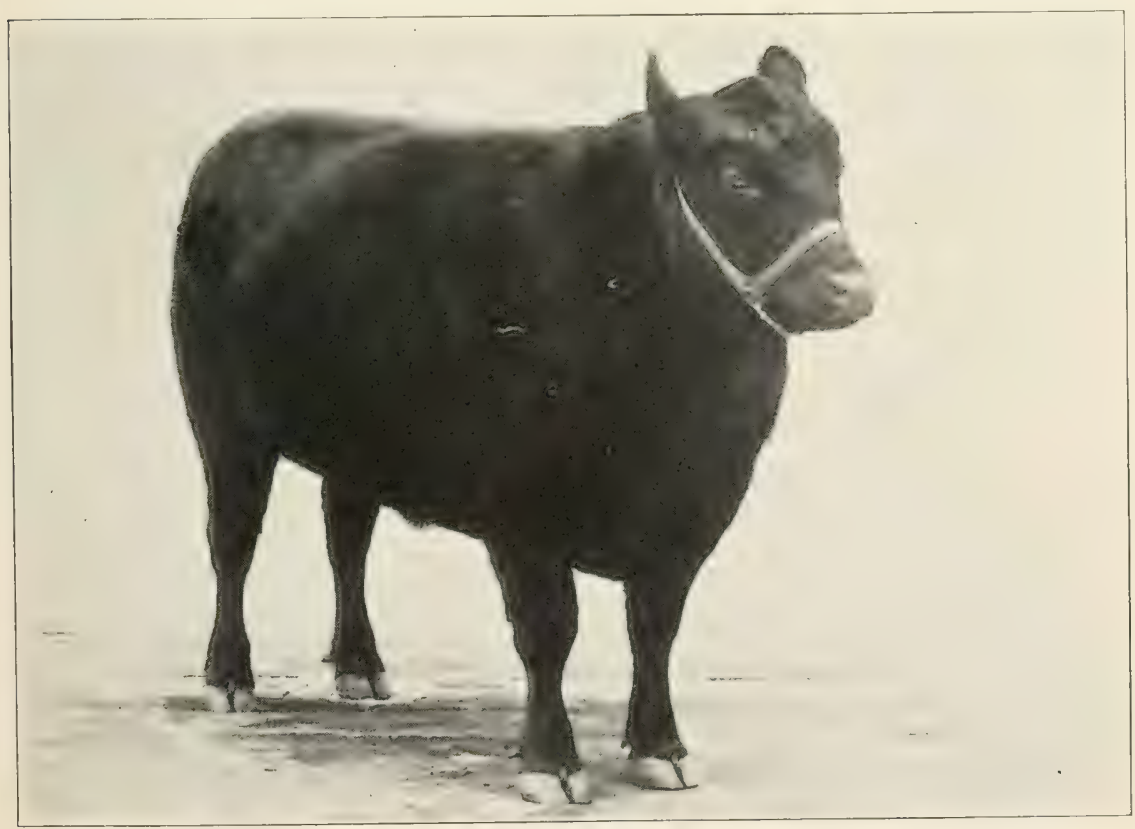

FYVIE KNIGHT

An Abeirdeen-Angus Produet of Indiana.

Stock Exposition Grand Champion Steer over all Breeds. Fect and Exhibited by Purdue Cniversity. 


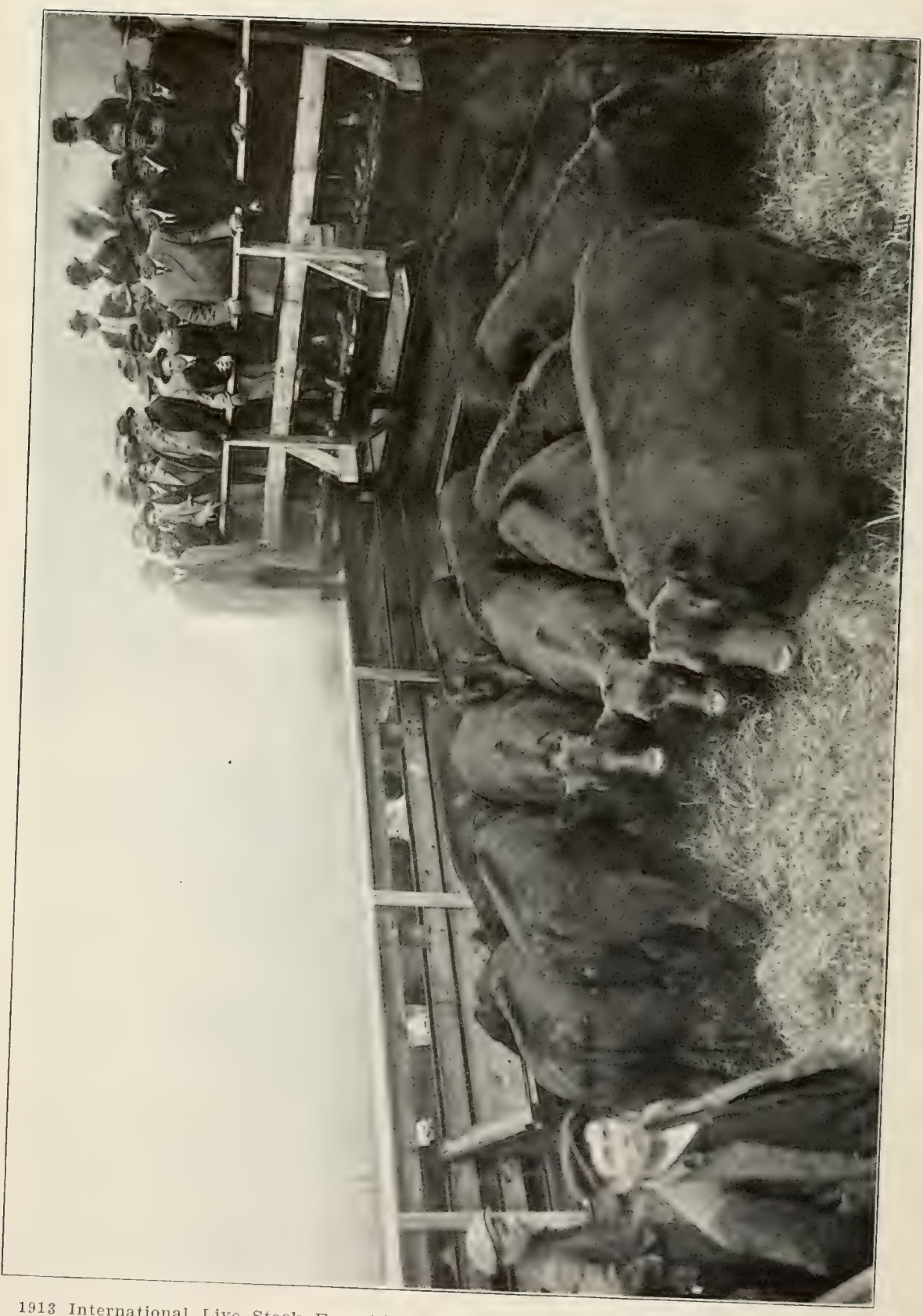

1913 International Live Stock Exposition Grand Champion Carload of Fat Steersover all Breeds. Fed and Exhibited by Escher \& Ryan. Fat Steers over 


\title{
Chicago International Live Stock Exposition Fat Carload Lot Awards
}

\author{
Competition Open to Steers and Heifers of Any Breed.
}

In the classification for Carload Lots the United States and Canada are divided into six Districts, and the territory which each District comprises is as follows:

Northwest District: Washington, Oregon, California, (north of the quarantine), Idaho, Nevada and Utah and the Territories and Provinces of Northwest Canada.

North Central District: Montana, Wyoming, North Dakota and South Dakota.

South Central District: Colorado and that part of the states of Kansas and Nebiaska lying west of the ninety-eighth degree of longtitude.

Southwest District: Arizona, New Mexico, Oklahoma and that part of Texas and Old Mexico lying north of the United States quarantine line.

Southern District: All that part of the United States and Mexico lying south of the United Stales quarantine line.

Eastern District: Animals to compete under this head may have been bred in any part of the world not provided for in the foregoing five districts.

1900

Total and No.

\section{of Entries}

of each Breed. District.

\author{
Class.
}

Aberdeen- Short- Here-

Angus horn ford Mixed
rating rating rating rating $\begin{array}{lllllll}123 & 123 & 123 & 123\end{array}$

1 Shorthorn: Northwest; 3 years and over............ 000

1 Aberdeen-Angus: North Central; 2 years and under $3 \ldots .100$

2 Both Mixed: North Central; 1 year and under 2........ 00 1 Hereford

21 Shorthorn: South Central; 3 years and over.......... 000 3 All Aberdeen-Angus: South Central; 2 years and under 3.1 2.3

3 All Herefords: Southwest; 3 years and over........... 000 2 Aberdeen-Angus

31 Hereford: Southwest: 2 years and under $3 \ldots \ldots \ldots 023$

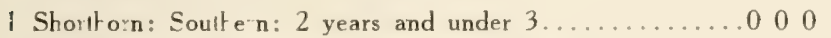

3 All Shorthorn: Southern; I year and under $2 \ldots \ldots \ldots 000$ 1 Aberdeen-Angus

1 Shorthorn

31 Hereford: Eastern; 3 years and over............. 03 1 Aberdeen-Angus

1 Hereford

31 Breed unknown: Eastern; 2 years and under 3..... 100

1 Hereford

32 Shorthorns: Eastern; 1 year and under $2 \ldots \ldots \ldots \ldots 00$

\section{Champion Fat Carloads by Ages}

3 All Herefords: 3 years or over................. $00000000123 \quad 000$ 1 Aberdeen-Angus

1 Hereford

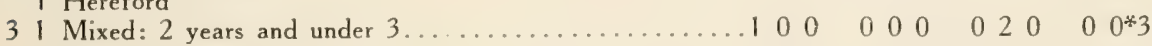
1 Aberdeen-Angus

1 Shorthorn

3 I Mixed: I year and under 2 . Grand Champion Carload of the Show-Aberdeen-Angus.

${ }^{*}$ Breed unknown. †Hereford-Shorthorn. $\begin{array}{llllllllll}1 & 0 & 0 & 0 & 0 & 0 & 0 & 0 & 0\end{array}$

$\begin{array}{lllllllll}0 & 0 & 0 & 0 & 0 & 0 & 0 & 0 & 0\end{array}$

$\begin{array}{llllllll}0 & 0 & 0 & 0 & 0 & 0 & 1 & 0\end{array}$

$020 \quad 1000000$

$\begin{array}{llllllllllllllll}0 & 0 & 0 & 0 & 0 & 0 & 0 & 0 & 0\end{array}$

$\begin{array}{llllllllll}0 & 0 & 0 & 1 & 2 & 3 & 0 & 0 & 0\end{array}$

$\begin{array}{lllllllll}0 & 0 & 0 & 1 & 0 & 0 & 0 & 0 & 0\end{array}$

$\begin{array}{lllllllll}1 & 0 & 0 & 0 & 0 & 0 & 0 & 0 & 0\end{array}$

123000000

$020 \quad 100 \quad 000$

$\begin{array}{llllllll}0 & 0 & 0 & 0 & 2 & 0 & 0 & 0\end{array}$

$1030200 \quad 000$

$000 \quad 0 \div 20$ 
Tolal ard No.

1901

of Eniries

of each Breed. District.

Class.

Aberdeen- Short- Here-

Angus horn ford Mixed

rating rating rating rating

$123 \quad 123 \quad 123 \quad 123$

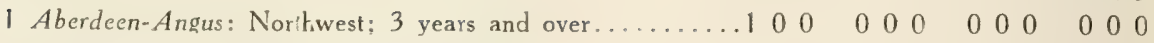

1 Hereford: Northwest; 2 years and under $3 \ldots \ldots \ldots \ldots 00000001000000$

1 Mixed: North Central; 3 years and over............ 000000000000100

2 Aberdeen-Angus

1 Hereford

41 Breed unknown: North Central; 2 years and under $3 \ldots 12000000003000$

1 Aberdeen-Angus

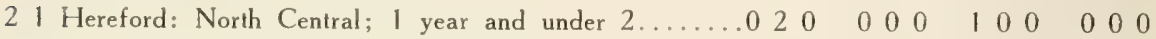

1 Hereford: South Central; 3 years and over............ 00000000100000

1 Aberdeen-Angus: South Central; 2 years and under 3....1 000000000000000

1 Hereford: Southwest; 3 years and over............ 00000000100000

1 Aberdeen-Angus

2 Herefords

52 Breed unknown: Southwest; 2 years and under $3 \ldots \ldots \ldots 003 \quad 00001200000$

1 Aberdeen-Angus

1 Hereford

1 Mixed

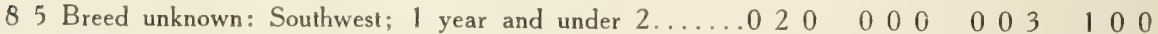

. Shorthorn: Southern; 3 years and over............. $000 \quad 1000000 \quad 000$

3 All Shorthorns: Southern; 2 years and under $3 \ldots \ldots \ldots .0001230000000$

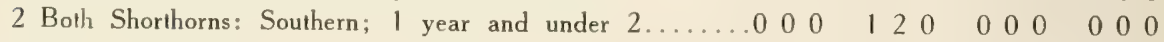

1 Aberdeen-Angus

1 Shorthorn

1 Mixed

52 Breed unknown: Eastern; 3 years and over......... 0201000000003

12 Aberdeen-Angus

219 Breed unknown: Eastern; 2 years and under $3 \ldots \ldots \ldots 12300000000000$

13 Aberdeen-Angus

Herefords

30 Shorthorns: Eastern; 1 year and under 2.

Number of Herefords and Shorthorns unknown.

$.0200003 \quad 1000000$

\section{Champion Fat Carloads by Ages}

1 Aberdeen-Angus

1 Hereford

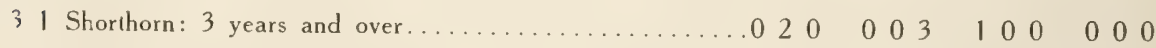

2 Aberdeen-Angus

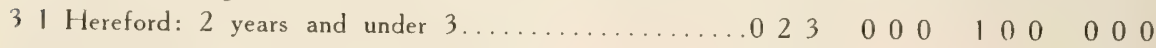

2 Herefords

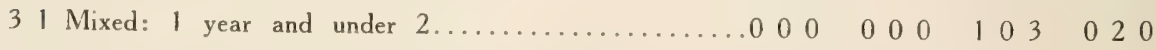

Grand Champion Carload of the Show-Herefords. 
1902

Total and No.

of Entries

of each Breed. District.

Class.

Aberdeen- Short- Here-

Angus horn ford Mixed rating rating rating rating

$123123123 \quad 123$

\section{Aberdeen-Angus}

21 Breed unknown: Norh Central; 2 years and under $3 \ldots .100000000000000$

3 All Aberdeen-Angus: North Central; 1 year and under 2..123 $3000 \quad 0000000$ 1 Aberdeen-Angus

21 Shorthorn: South Ceniral; 2 years and under $3 \ldots \ldots \ldots 02001000000000$

4 Herefords

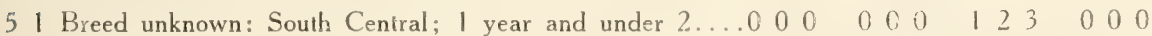

3 All Herefords: Southwest: 2 years and under $3 \ldots \ldots \ldots 0000000123000$

2 Both Herefords: Southwest; 1 year and under $2 \ldots \ldots \ldots 000000120000$

2 Both. Shorthorns: Southern; 3 years and over.......... 0001201000000 Mixed Load

- Shorhorn and Hereford: Southern; 2 years and under 3..0 000 C $000 \quad 0000100$

3 Herefords

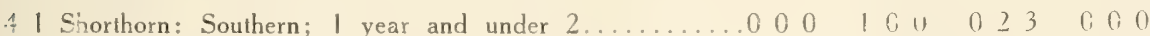

4 Aberdeen-Angus

3 Herefords

4 Shorthorns

121 Mixed: Eastern: 3 years and over............... $20010 € 003000$

16 Aberdeen-Angus

11 Herefords

12 Shorthorns

423 Breed unknown: Easiern; 2 years and under $3 \ldots \ldots \ldots 10300200000000$

15 Aberdeen-Angus

7 Herefords

5 Shorthorns

336 Breed unknown: Eastern; I year and under 2.......020 20 G C 00103000

*Bieed unknown.

\section{Champion Fat Carloads by Ages}

2 Both Shorthorns: 3 years and over............... $000 \quad 1200000000$

\section{Aberdeen-Angus}

1 Hereford

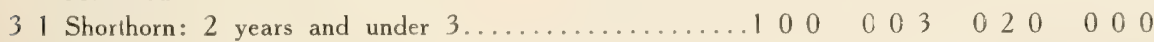

1 Aberdeen-Angus

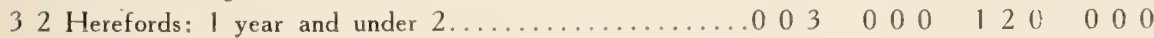

$\mathrm{G}_{1}$ and Champion Carload of the Show-Aberdeen-Angus. 
Total and No.

1903

of Entries

of each Breed. District.

Class.

Aberdeen- Shori- Here-

Angus horn ford

Mixed

rating rating rating rating

123

2 Both Aberdeen-Angus: North Central; 2 y'rs and under 3..12 0

1 Hereford: South Central; 3 years or over............. 00

9 Breed unknown: South Central; 2 years and under 3...0 03

5 Breed unknown: South Central; 1 year and under 2.....0 03

1 Aberdeen-Angus

21 Shorthorn: Southwest; 3 years or over............. 20

5 Breed unknown: Southwest; 2 years and under 3.......0 03

6 All Herefords: Southwest; I year and under 2....... 000

1 Hereford: Southern; 3 years or over.............. 00

1 Hereford: Southern; 1 year and under $2 \ldots \ldots \ldots \ldots . \ldots 00$

123123

123

$\begin{array}{lllllllllllllllll}0 & 0 & 0 & 0 & 0 & 0 & 0 & 0 & 0\end{array}$

$\begin{array}{lllllllllllll}0 & 0 & 0 & 1 & 0 & 0 & 0 & 0 & 0\end{array}$

$\begin{array}{llllllllll}0 & 0 & 0 & 1 & 2 & 0 & 0 & 0 & 0\end{array}$

$\begin{array}{lllllllll}0 & 0 & 0 & 1 & 0 & 0 & 0^{*} 2 & 0\end{array}$

2 Aberdeen-Angus

1 Shorthorn

2118 Breed unknown: Eastern; 3 years or over.......... 23

38 Breed unknown: Eastern; 2 years and under $3 \ldots \ldots \ldots .103$

Breed unknown: Eastern; 1 year and under $2 \ldots \ldots \ldots . . .000$

*A load of yearling Galloways won second prize in South Central District.

10000000000

$\begin{array}{llllllllll}0 & 0 & 0 & 1 & 2 & 0 & 0 & 0 & 0\end{array}$

$\begin{array}{llllllllll}0 & 0 & 0 & 1 & 2 & 3 & 0 & 0 & 0\end{array}$

$\begin{array}{llllllllllllllll}0 & 0 & 0 & 1 & 0 & 0 & 0 & 0 & 0\end{array}$

$\begin{array}{lllllllll}0 & 0 & 0 & 1 & 0 & 0 & 0 & 0 & 0\end{array}$

2 Herefords

Champion Fat Carloads by Ages

31 Shorthorn: 3 years or over.................... 00

1 Aberdeen-Angus

32 Herefords: 2 years and under $3 \ldots \ldots \ldots \ldots \ldots \ldots$

3 All Herefords: 1 year and under $2 \ldots \ldots \ldots \ldots \ldots \ldots$

Grand Champion Carload of the Show-Herefords.

1000023000

$\begin{array}{llllllllllllll}0 & 0 & 0 & 0 & 2 & 3 & 0 & 0 & 0\end{array}$

$\begin{array}{lllllllll}0 & 0 & 0 & 1 & 2 & 3 & 0 & 0 & 0\end{array}$

1904

3 All Herefords: North Central; 3 years or over.........00 00 1 Aberdeen-Angus

32 Herefords: South Central; 1 year and under 2......020 1 Aberdeen-Angus

54 Herefords: Southwest; 2 years and under 3....... 20

3 All Herefords: Southwest; 1 year and under 2....... 000

1 Shorthorn: Southern; 3 years or over............... 00

2 Both Herefords Southern; 1 year and under 2....... 000

3 All Shorthorns: Eastern: 3 years or over............ 000

$\begin{array}{lllllllllll}0 & 0 & 0 & 0 & 0 & 0\end{array}$

$\begin{array}{llllllllll}0 & 2 & 0 & 0 & 0 & 0 & 0 & 0 & 0\end{array}$ $\begin{array}{lllllllll}0 & 2 & 3 & 1 & 0 & 0 & 0 & 0 & 0\end{array}$

8 Aberdeen-Angus

1 Hereford

101 Shorthorn: Eastern; 2 years and under 3.

6 Aberdeen-Angus

8 Herefords

195 Shorthorns: Eastern; 1 year and under 2........ 033000

$020 \quad 000$

\section{Champion Fat Carloads by Ages}

Breed unknown: 3 years or over

100

$\begin{array}{llllllll}0 & 0 & 3 & 0 & 0 & 0\end{array}$

9 Aberdeen-Angus

8 Herefords

7 Aberdeen-Angus

12 Herefords

245 Shorthorns: 1 year and under 2.

Grand Champion Carload of the Show-Aberdeen-Angus.

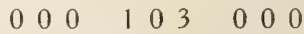

$\begin{array}{llllllllll}0 & 0 & 0 & 1 & 2 & 3 & 0 & 0 & 0\end{array}$

$\begin{array}{llllllllll}1 & 0 & 0 & 0 & 0 & 0 & 0 & 0 & 0\end{array}$

$\begin{array}{llllllllll}0 & 0 & 0 & 1 & 2 & 0 & 0 & 0 & 0\end{array}$

$\begin{array}{llllllll}1 & 23 & 0 & 0 & 0 & 0 & 0 & 0\end{array}$

$\begin{array}{lll}0 & 0 & 0\end{array}$

$\begin{array}{llllll}0 & 23 & 0 & 0 & 0\end{array}$ 
1905

Total and No.

of Entries

of each Breed. District.

Class.

Aberdeen- Short- Hele-

Angus horn ford

rating rating rating

Mixed

1 Hereford: North Central; 3 years and over........... 0000000001000000

1 Hereford: North Central; 2 years and under $3 \ldots \ldots \ldots .00000000100000$

2 Both Hereford: North Central; 1 year and under $2 \ldots \ldots 000000001200000$

I Shorthorn: South Central; 3 years and over.......... $0000 \quad 10000000000$

2 Herefords

31 Galloway: South Central; 2 years and under $3 \ldots \ldots .00000000120000 \% 3$

2 Aberdeen-Angus

2 Herefords

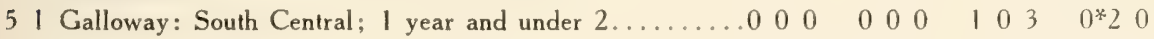

1 Hereford: Southwest; 3 years and over.............. 00000000100000

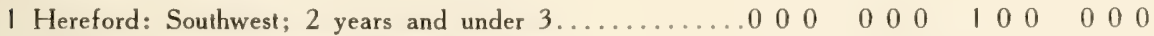

7 All Herefords: Southwest; 1 year and under 2....... 00000000123000

\section{Hereford}

21 Shorthorn: Southern: 3 years and over............ 00000200100000

4 Herefords: Southern; 2 years and under $3 \ldots \ldots \ldots \ldots 0000000123000$

4 Herefords

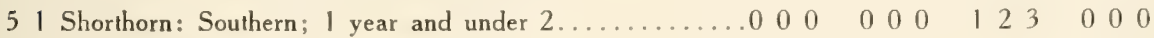

2 Aberdeen-Angus

2 Herefords

84 Shorthorns: Eastern; 3 years and over........... 0200003100000

8 Aberdeen-Angus

1 Hereford

101 Shorthorn: Eastern; 2 years and under $3 \ldots \ldots \ldots 1230 \ldots 000000000$

7 Aberdeen-Angus

2 Herefords

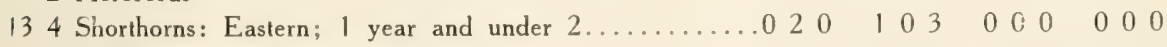

*A load of yearling Galloways won second prize in South Central District; also third prize in same District in 2-year-old class.

\section{Champion Fat Carloads by Ages}

3 All Herefords: 3 years and over................ 00000000123000

1 Aberdeen-Angus

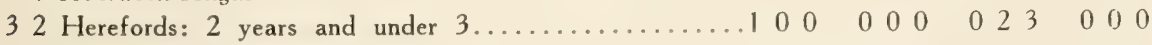

2 Herefords

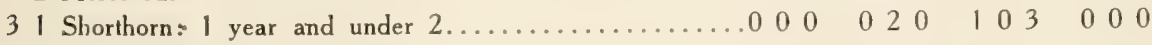

Grand Champion Carload of the Show-Aberdeen-Angus. 
Total and No.

of Eniries

of each Breed. District.
Aberdeen- Short- Here-

Angus horn ford Mixed

Class.

rating rating rating rating

$\begin{array}{lllllllll}123 & 123 & 123 & 123\end{array}$

2 Both Herefords: North Central; 2 years and under 3...000 0

2 Both Herefords: North Central: I year and under 2...000

Heleford: South Ceniral: 3 years and over............000

2 Both Herefords: South Central; 2 years and under 3...000

$0000 \quad 120 \quad 000$

$\begin{array}{lllllllll}0 & 0 & 0 & 1 & 2 & 0 & 0 & 0 & 0\end{array}$

$\begin{array}{lllllllll}0 & 0 & 0 & 1 & 0 & 0 & 0 & 0 & 0\end{array}$

$000 \quad 120 \quad 000$

2 Aberdeen-Angus

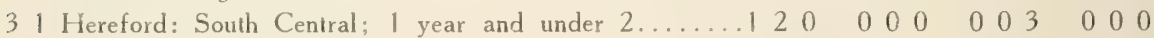

2 Herefords

31 Shorhorn: Southwest; 2 years and under $3 \ldots \ldots \ldots \ldots 00000200103000$

7 Herefords

81 Shorthorn: Southwest; 1 year and under $2 \ldots \ldots \ldots \ldots .0000100002300$

3 All Herefords: Southern; I year and under 2....... 000000000123000

6 Aberdeen-Angus

3 Herefords

3 Sinorthorns

13 I Galloway: Eastern: 3 years and over...........1000 000300200000

10 Aberdeen-Angus

4 Herefords

!5 1 Shorthorn: Eastern; 2 years and under $3 \ldots \ldots \ldots \ldots 123 \quad 0 \ldots 000000000$

7 Aberdeen-Angus

2 Herefords

10 Shorthorns

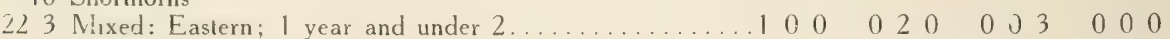

\section{Champion fat Carloads by Ages}

\section{Aberdeen-Angus}

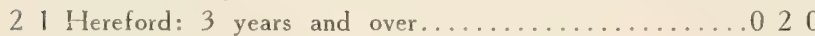

$0000 \quad 1000 \quad 000$

1 Aberdeen-Angus

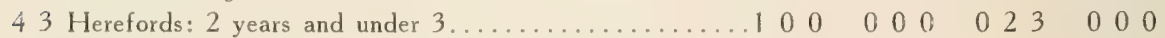

2 Aberdeen-Angus

2 Herefords

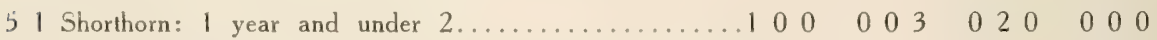
Grand Champion Carload of the Show-Aberdeen-Angus. 
Toral and No.

of Entries

of each Breed. District. Class.

Aberdeen- Short- Here-

Angus horn ford Mixed

rating rating rating rating

$123123 \quad 123 \quad 123$

4 All Herefords: North Central; 3 years or over......... 00000000123000

1 Hereford: North Central; 2 years and under $3 \ldots \ldots \ldots \ldots 00000000100000$

2 Herefords

3 Sliorthorn: North Central; 1 year and under $2 \ldots \ldots \ldots 000 \quad 1000023000$

3 Herefords

I Shorthorn

51 Aberdeen-Angus: South Central; 3 years or over.....0000 $003 \quad 1200000$

3 Herefords

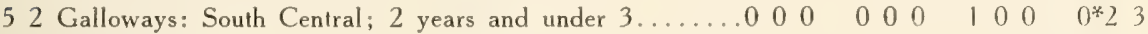

6 Herefords

82 Aberdeen-Angus: South Central; 1 year and under 2..0 0000000123000

2 Both Herefords: Southwest; 3 years or over......... 00000000120000

1 Hereford: Southwest; 2 years and under $3 \ldots \ldots \ldots .000000000100000$

8 Herefords

102 Shorthorns: Southwest; 1 year and under $2 \ldots \ldots \ldots \ldots 0001000023000$

2 Both Herefords: Southern; 2 years and under $3 \ldots \ldots \ldots .000000001200000$

2 Both Herefords: Southern; 1 year and under $2 \ldots \ldots \ldots \ldots 000000001200000$

4 Aberdeen-Angus

4 Shorthorns

124 Herefords: Eastern; 3 years or over............ 0200003100000

13 Aberdeen-Angus

3 Herefords

171 Shorthorn: Eastern; 2 years and under $3 \ldots \ldots \ldots \ldots 12000003000000$

6 Aberdeen-Angus

10 Herefords

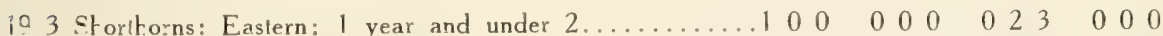

*Galloways won second and third prizes in 2-year-old class in South Central District.

\section{Champion Fat Carloads by Ages}

4 All Herefords: 3 years or over................. 00000000123000

1 Aberdeen-Angus

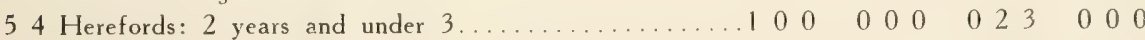

1 Aberdeen-Angus

2 Herefords

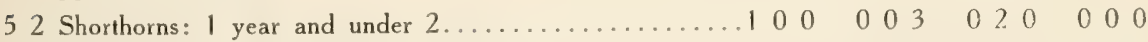

Grand Champion Carload of the Show-Aberdeen-Angus. 
1908

Total and No.

of Entries

of each Breed. District.

Class.

Aberdeen- Short- Here-

Angus horn ford Mixed

rating rating rating rating

$123123 \quad 123 \quad 123$

3 Herefords

41 Galloway: North Central; 2 years and under $3 \ldots \ldots .00000000123000$

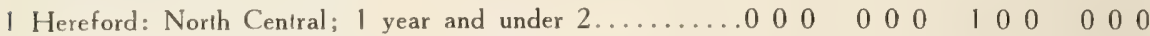

1 Hereford: South Central; 3 years or over............ 000000000100000

2 Both Herefords: South Central; 2 years and under 3... 000000001200000

3 Aberdeen-Angus

52 Herefords: South Central; 1 year and under $2 \ldots \ldots \ldots 103000000200000$

2 Both Herefords: Southwest; 2 years and under 3......0 000000001200000

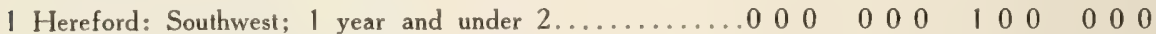

2 Both Herefords: Southern; 2 years and under $3 \ldots \ldots \ldots 00000001200000$

1 Hereford: Southern; 1 year and under $2 \ldots \ldots \ldots \ldots .000000001000000$

2 Aberdeen-Angus

3 Shorthorns

2 Herefords

81 Mixed: Eastern; 3 years or over...............

9 Aberdeen-Angus

101 Hereford: Eastern; 2 years and under $3 \ldots \ldots \ldots \ldots 1230 . \ldots 0001000000$

5 Aberdeen-Angus

3 Shorthorns

7 Herefords

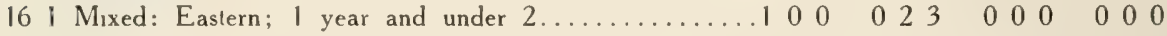

\section{Champion Fat Carloads by Ages}

1 Aberdeen-Angus

21 Hereford: 3 years or over.................. 000000000200000

1 Aberdeen-Angus

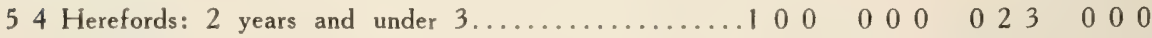

2 Aberdeen-Angus

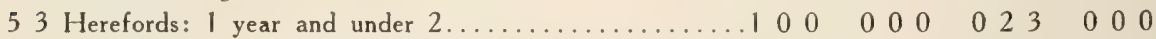

Grand Champion Carload of the Show-Aberdeen-Angus. 


\section{9}

Total and No.

of Entries

of each Breed. District.

\begin{tabular}{|c|c|c|c|}
\hline . & $\begin{array}{c}\text { Aberdeen- } \\
\text { Angus } \\
\text { rating }\end{array}$ & $\begin{array}{l}\text { Short- } \\
\text { horn } \\
\text { rating }\end{array}$ & $\begin{array}{l}\text { Here- } \\
\text { ford } \\
\text { rating }\end{array}$ \\
\hline & 123 & 123 & 123 \\
\hline & $\begin{array}{llll}0 & 0 & 0\end{array}$ & 000 & 100 \\
\hline & .000 & 000 & 100 \\
\hline
\end{tabular}

1 Hereford: North Central; 3 years and over

I Hereford: South Central; 3 years and over............ 00

3 Herefords

52 Shorthorns: South Central; 2 years and under $3 \ldots \ldots 0000003120000$

1 Shorthorn: South Central; 1 year and under $2 \ldots \ldots \ldots \ldots 000010000000000$

3 All Herefords: Southwest; 3 years and over......... 0000000123000

6 All Herefords: Southwest: 2 years and under $3 \ldots \ldots \ldots 00000000123000$

1 Aberdeen-Angus

2 Herefords

41 Shorthorn: Southwest; 1 year and under $2 \ldots \ldots \ldots \ldots .003 \quad 10000200000$

3 Aberdeen-Angus

2 Herefords

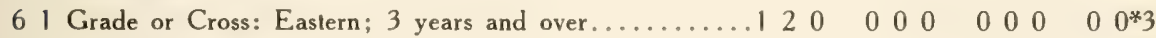

7 Aberdeen-Angus

81 Shorthorn: Eastern; 2 years and under $3 \ldots \ldots \ldots .12300000000000$

10 Aberdeen-Angus

5 Herefords

227 Shorthorns: Eastern; 1 year and under $2 \ldots \ldots \ldots .023 \quad 10000000000$

\section{Champion Fat Carloads by Ages}

\section{Aberdeen-Angus}

43 Herefords: 3 years and over................... 030000120000

1 Aberdeen-Angus

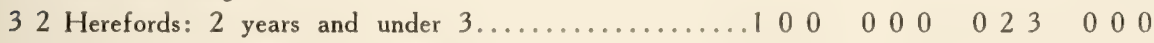

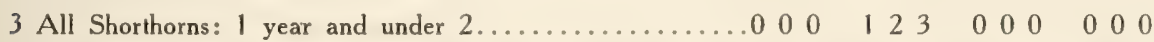

Grand Champion Carload of the Show-Shorthorn. Reserve Grand Champion CarloarlAberdeen-Angus.

* Grade or Cross. Breed unknown. 
Total and No.

of Eniries

of each breed.

District.

Class.

Aberdeen- Short- Here- Collo- Mixed

1 Shorthorn

Angus horn ford way Cross-

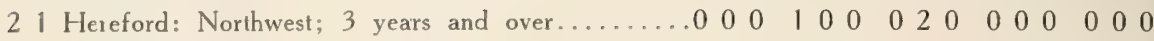

3 Herefords

41 Shorthorn: North Central; 3 years and over......0 000000012300000000

2 All Herefords: South Central; 3 years and over....0 0000000012000000000

5 Aberdeen-Angus

3 Shorthorns

102 Herefords: South Central; 2 years and under $3 \ldots 12330000000000000000$

2 Aberdeen-Angus

2 Shorthorns

51 Galloway: South Central; 1 year and under $2 \ldots \ldots 10000023000000000000$

3 All Herefords: Southwest; 2 years and under $3 \ldots \ldots 0000000012300000000$

2 Aberdeen-Angus

64 Hereford: Southwest; 1 year and under $2 \ldots \ldots \ldots 0233000010000000000$

7 Aberdeen-Angus

5 Shorthorns

2 Herefords

151 Mixed He efo:d-Shorthorn: Eastern; 3 yrs. and over.1 2000000000030000000

4 Aberdeen-Angus

4 Shorthorns

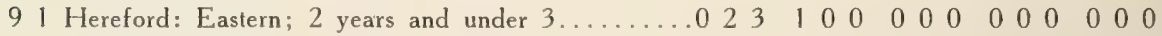

9 Aberdeen-Angus

5 Shorhorns

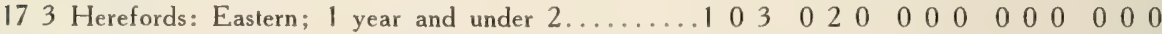

1 Aberdeen-Angus

Champion Fat Carloads by Ages

32 Herefords: 3 years and over..............

1 Aberdeen-Angus

1 Shorthorn

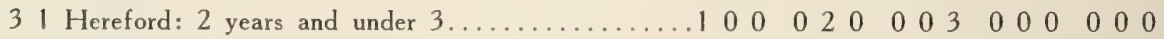

2 Aberdeen-Angus

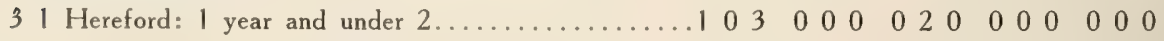

Grand Champion Fat Carload of the Show-Aberdeen-Angus.

Reserve Grand Champion Carload of the Show-Aberdeen-Angus.

\section{Short Fed Fat Carload Awards}

3 Aberdeen-Angus

1 Shorthorn

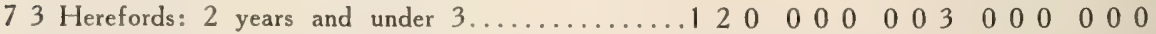

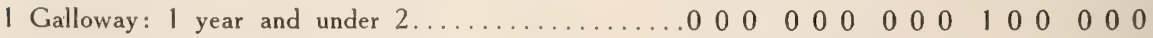

Grand Champion Short Fed Fat Carload of the Show-Aberdeen-Angus. 
Tolal and No.

of Entries

of Each Breed.

District. Class.

Aberdeen- Short- Here-

Angus horn ford

rating rating rating

123123123

2 Both Herefords: North Central: 3 years and over........... 00000000120

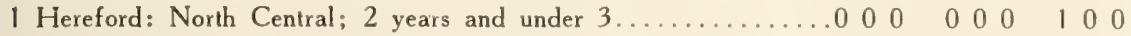

2 Both Herefords: North Central; 1 year and under $2 \ldots \ldots \ldots .0000000120$

3 Herefords

1 Galloway

51 Shorthorn: South Central; 2 years and under $3 \ldots \ldots \ldots \ldots \ldots 0000003120$

4 Shorthorns

84 Herefords: South Central; 1 year and under $2 \ldots \ldots \ldots \ldots . .00000000123$

2 Both Herefords: Southwest; 3 years and over............... $00000000 \quad 120$

1 Shorthorn

32 Herefords: Southwest; 2 years and under $3 \ldots \ldots \ldots \ldots \ldots \ldots 00010 \ldots 00023$

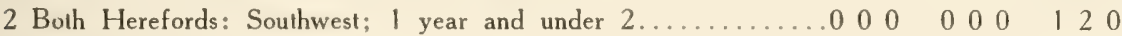

1 Shorthorn

54 Aberdeen-Angus: Eastern; 3 years and over............. 1200003000

2 Shorthorns

1 Hereford

118 Aberdeen-Angus: Eastern; 2 years and under $3 \ldots \ldots \ldots \ldots \ldots 1200003000$

4 Shorthorns

1 Hereford

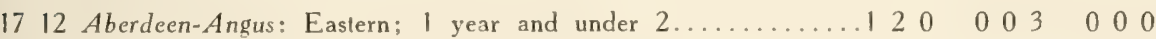

\section{Champion Fat Carloads by Ages}

2 Herefords

31 Aberdeen-Angus; 3 years and over.................. 00000000023

1 Shorthorn

1 Hereford

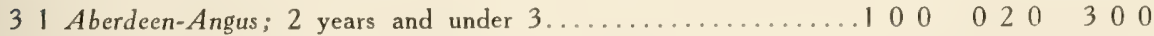

2 Herefords

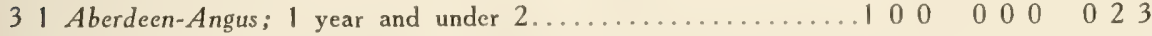

Grand Champion Fat Carload of Show-Aberdeen-Angus.

Reserve Grand Champion Fat Carload of the Show-Aberdeen-Angus. 
Total and No.

of Entries

of Each Breed.

District. Class.

Aberdeen- Shorl- Here-

Angus horn ford

rating rating rating

$123123 \quad 123$

1 Hereford

21 Aberdeen-Angus: North Central; 1 year and under $2 \ldots \ldots \ldots .02000000100$

1 Hereford: South Central; 3 years and over............... 00000000100

2 Aberdeen-Angus

2 Herefords

62 Shorthorns: South Central; 2 years and under $3 \ldots \ldots \ldots \ldots 10000030020$

4 Aberdeen-Angus

4 Herefords

113 Shorthorns: South Central; 1 year and under $2 \ldots \ldots \ldots \ldots \ldots 12000000003$

4 Herefords

51 Shorthorn: Southwest; 2 years and under $3 \ldots \ldots \ldots \ldots \ldots \ldots 000010 \ldots 0023$

1 Hereford

32 Aberdeen-Angus: Eastern; 3 years and over.............. 02300000100

2 Shorthorns

75 Aberdeen-Angus: Eastern; 2 years and under $3 \ldots \ldots \ldots \ldots \ldots 1230 \ldots 000000$

17 Aberdeen-Angus

4 Shorthorns

1 Galloway

275 Herefords: Eastern; 1 year and under $2 \ldots \ldots \ldots \ldots \ldots \ldots \ldots 12000030 . \ldots 00$

\section{Champion Fat Carloads by Ages}

2 Herefords: 3 years and over...................... 000000000120

1 Shorthorn

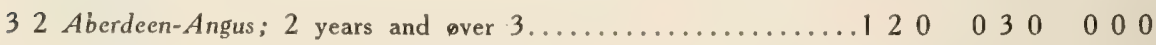

3 Aberdeen-Angus; 1 year and under $2 \ldots \ldots \ldots \ldots \ldots \ldots \ldots 230 \ldots \ldots$

Grand Champion Fat Carload of the Show-Aberdeen-Angus.

Reserve Grand Champion Fat Carload of the Show-Aberdecn-Angus. 
1913

Jotal and Number

Each Breed. District. Class. of Enirics of

Aberdeen- Short- Here- Gallo-

Angus horn fo:d way Mixed rating. rating. ra'ing. raling. rating.

$123123 \quad 123 \quad 123 \quad 123$

2 Herefords: North Central; 3 years and over. 00000000012000000000

2 Herefords

42 Shcrthorns: North Central; 2 years and under $3.0000003 \quad 12000000000$

1 Hereford

21 Galloway: South Central; 3 years and over...0 00040000100010200000

4 Herefords

73 Mixed: South Central: 2 years and under $3 \ldots 000000000230000100$

3 Aberdeen-Angus

1 Shorthorn

62 Herefords: South Central; 1 year and under 2.10030020000000000000

1 Hereford: Souttwest; 3 years and over........ 0000000001000000000

1 Shorthorn

43 Herefords: Southwest: 2 years and under $3 \ldots 0000000001230000000$ 3 Herefords: Southwest; 1 year and under $2 \ldots 000000001230000000$

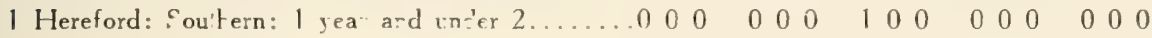

6 Aberdeen Angus

5 Herefords

I Shorthorn

1 Galloway

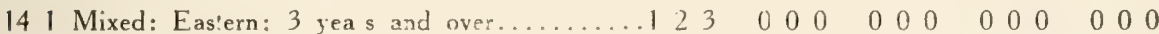

9 Aberdeen-Angus

3 Shorthorns

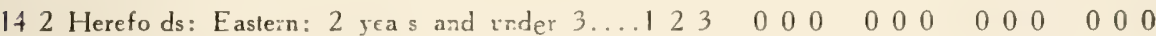

9 Aberdeen-Angus

4 Storttorn

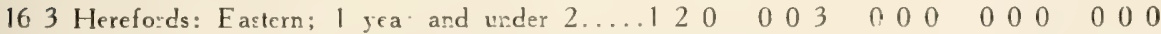

3 Herefords

\begin{tabular}{|c|c|}
\hline $\begin{array}{l}\text { berdeen- } \\
\text { Angus } \\
\text { rating }\end{array}$ & $\begin{array}{c}\text { Short- } \\
\text { horn } \\
\text { rating }\end{array}$ \\
\hline 123 & 123 \\
\hline
\end{tabular}

41 Aberdeen-Angus; 3 years and over.................. 0 . 0000000023

2 Hierefords

I Mixed

41 Aberdeen-Angus; 2 years and over.................

2 Herefords

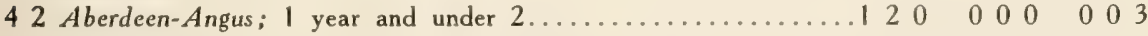

Grand Champion Fat Carload of the Show-Aberdeen-Angus.

Reserve Grand Champion Fat Carload of the Show-Aberdeen-Angus. 


\section{Summary of Fat Carload Lot Competition}

Grand Champion Fat Carload lot Awards

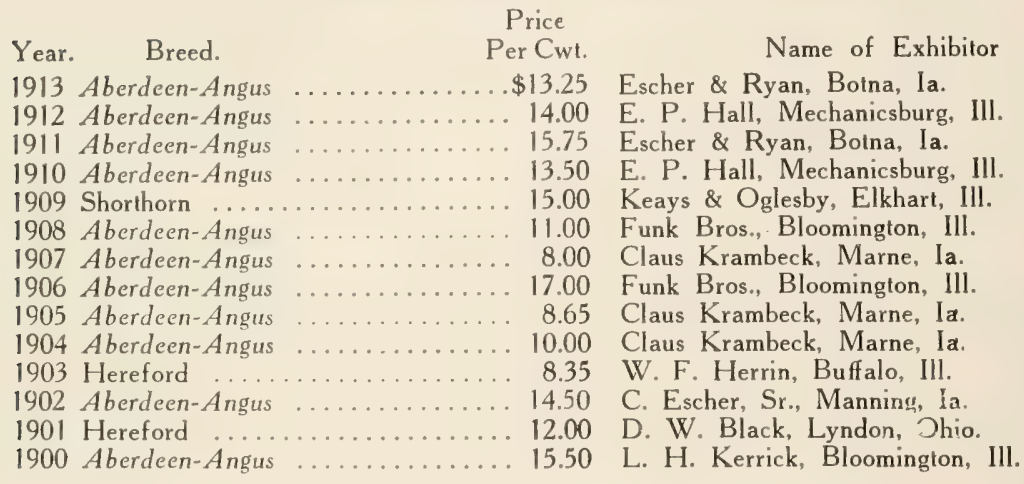

\section{Chicago International Live Stock Exposition}

Comparative Average Prices of Fat Carload Lots at Auction by Breeds

$\begin{array}{lllll}1913 & 1912 & 1911 & 1910 & 1909\end{array}$

No. Av. No. Av. No. Av. No. Av. No. Av.

NAME OF BREED. loads price loads price loads price loads price loads price

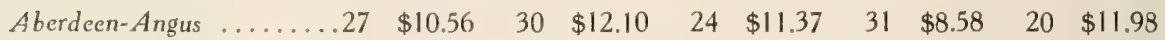

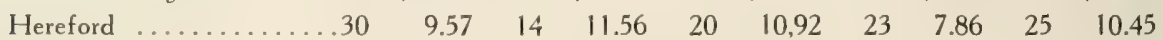

$\begin{array}{lllllllllllll}\text { Shorthorn } \ldots \ldots \ldots \ldots \ldots .13 & 9.98 & 12 & 11.63 & 13 & 10.68 & 23 & 8.10 & 13 & 11.24\end{array}$

Galloway $\ldots \ldots \ldots \ldots \ldots .3 \quad 9.28 \quad 1 \quad 11,10 \quad 1 \quad 10.75 \quad 1 \quad 7.80$

$1908 \quad 1907 \quad 1906 \quad 1905 \quad 1904$

No. Av. No. Av. No. Av. No. Av. No. Av.

NAME OF BREED, loads price loads price loads price loads price loads price

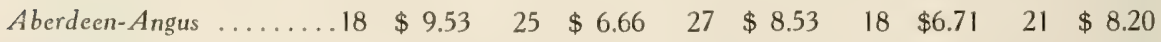

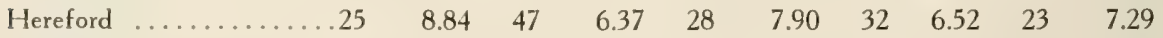

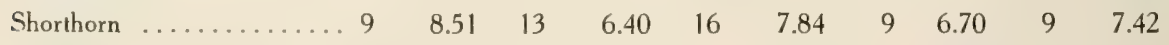

Galloway $\ldots \ldots \ldots \ldots \ldots 1 \quad 8.00 \quad 5 \quad 6.51 \quad 1 \quad 7.75 \quad 1 \quad 6.80$

Mixed, Texans and cows..

1903

1902

1901

1900

$1900-1913$

No. Av. No. Av. No. Av. No. Av. No. Av.

NAME OF BREED. loads price loads price loads price loads price loads price

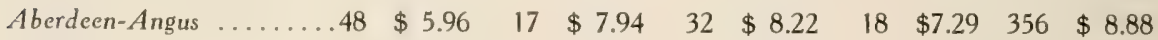

$\begin{array}{lllllllllll}\text { Hereford } \ldots \ldots \ldots \ldots \ldots .24 & 5.99 & 11 & 7.48 & 22 & 8.23 & 17 & 6.29 & 341 & 8.07\end{array}$

$\begin{array}{lllllllllll}\text { Shorthorn } \ldots \ldots \ldots \ldots \ldots .23 & 5.61 & 11 & 7.45 & 10 & 7.74 & 13 & 6.25 & 187 & 8.22\end{array}$

Cialloway ........... $2 \quad 6.05 \quad 2 \quad 6.45 \quad 18 \quad 7.68$

$\begin{array}{llllllllll}\text { Mixed, Texans and cows. } 22 & 5.64 & 2 & 7.22 & 12 & 7.60 & 1 & 6.00 & 51 & 6.32\end{array}$ 


\section{Summary of Fat Carload Lot Competition}

The foregoing account of sales of carload lois at auction shows that during a period of fourteen years the Aberdeen-Angus have made an average of $81 \mathrm{c}=$ nts per cwt. more than the Herefords and 66 cents more than the Shorthorns. These figures obtained from such a practica! source show the degree in which the Aber. deen-Angus leads its rivals in establishing average top prices.

A careful review of awards for fourteen years shows that the 'eading breeds were well represented.

The feature, or point, however, that should interest and appeal not only to Aberdeen-Angus breeders but also breeders of all breeds and cattle feeders in general, is how much actual competition each breed had during the period of fourteen years.

An analysis of the awards sets forth the following facts:

Out of the total number of four hundred and twenty-three awards Aberdeen-Angus won forty-three firsts, forty-seven seconds and thirty-one thirds; Herefords ninety firsts, sixty-three seconds and forty-two thirds; Shorthorns, twentyeight firsts, eighteen seconds and forty-four thirds; Galloways one first, four seconds and two thirds; mixed, five firsts, two seconds and three thirds.

The interesting feature of the Fat Carload Lot competition is to learn how many prizes each breed won where its rivals were not represented. We find that Aberdeen-Angus won seven firsts, four seconds and thrce thirds where no Hereford, Shorthorns, etc., were shown. The Shorthorns won nineteen firsts, nine seconds and seven thirds where no Aberdeen-Angus were shown. Herefords won seventy firsts, fifty-two seconds and twenty-seven thirds where Aberdeen-Angus were absent. By making the proper deductions from the actual awards and only taking into consideration the classes in which Aberdeen-Angus competed with Herefords or Shorthorns or both or other breeds, we find that the Aberdeen-Angus won thirty-seven firsts, forty-two seconds and twenty-eight thirds; Herefords, twenty-one firsts, twelve seconds and fourteen thirds; Shorthorns, nine firsts, nine seconds and fifteen thirds.

It will be noted in the competition for champions by ages there were one hundred and twenty-three possible prizes and the representation won by each breed for this competition by virtue of its winnings in the open district classes is as follows: Aberdeen-Angus, represented in thirty-nine classes; Herefords, fortynine c'asses; Shorthorns, twenty-one classes. While the Herefords. secured thirteen more chances than the Aberdeen-Angus, the results show that Aberdeen-Angus won twenty-six firsts, nine seconds and nine thirds; Herefords, nineteen firsts, thirty-one seconds and twenty-five thirds; Shorthorns, five firsts, six seconds and eight thirds; mixed, one first, two seconds and one third.

While deductions from the above facts show clearly that the AberdeenAngus is superior, as a climax it is only neceisary to add that out of fourteen possible Grand Championships offered for best carload, Aberdeen-Angus have won eleven times, Herefords twice and Shorthorn once. 


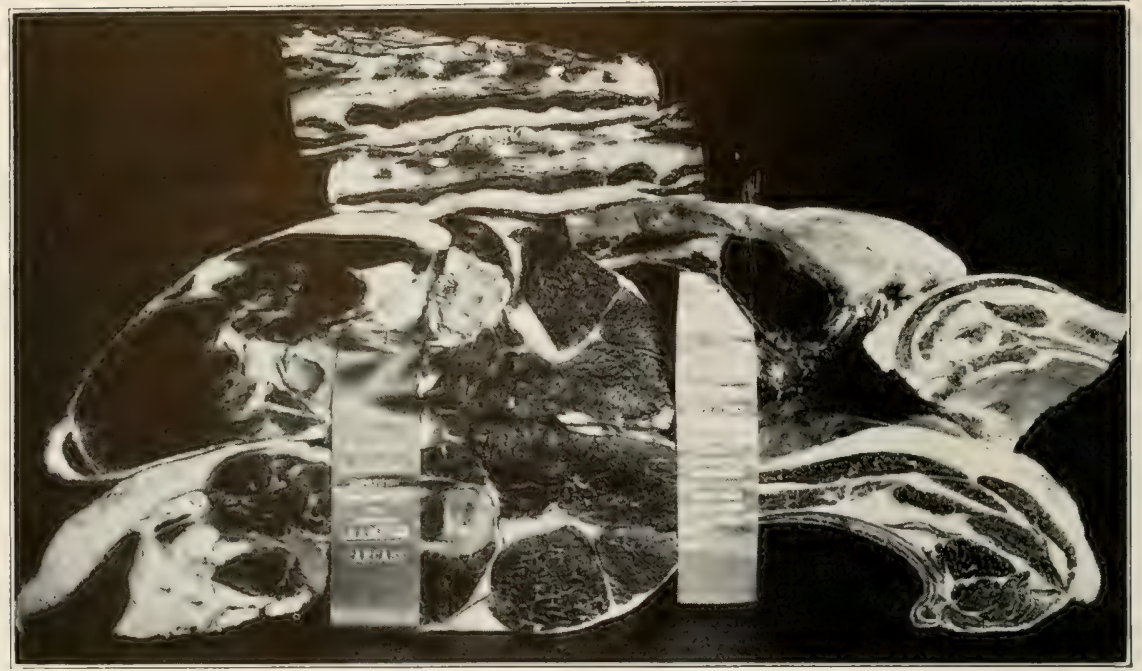

AN ABERDEEN-ANGUS PRODUCT OF PENNSYLVANIA.

Cuts of 1910 Grand Champion Carcass over all Breeds at International Live Stock Exposition. Bred, Fed and Exhibited by C. L. Taggart, Washington, Pennsylvania.

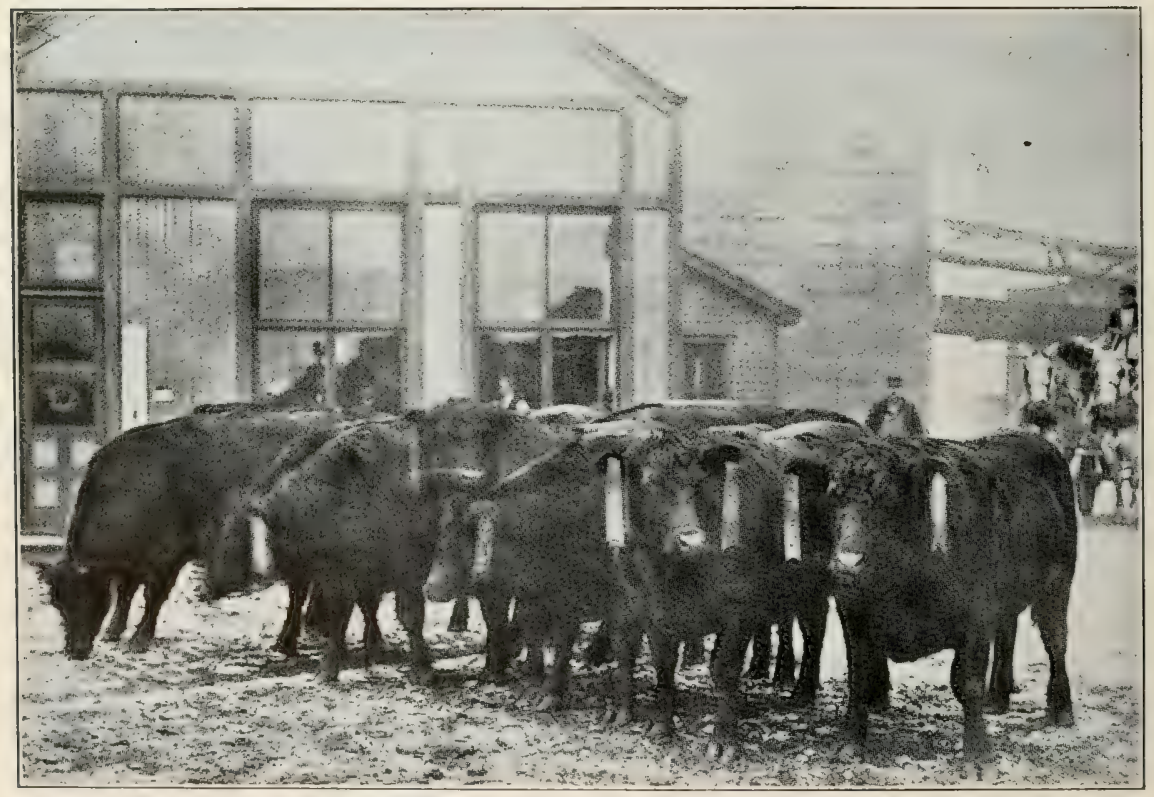

\section{AN ABERDEEN-ANGUS PRODUCT OF IOWA.}

Grand Champion Carload of Fat Steers over all Breeds at Pittsburgh Fat Stock show. Bred, Fed and Exhibited by Charles Escher, Sr., and Sold at World's Record Price, 211/2c Per Pound. 


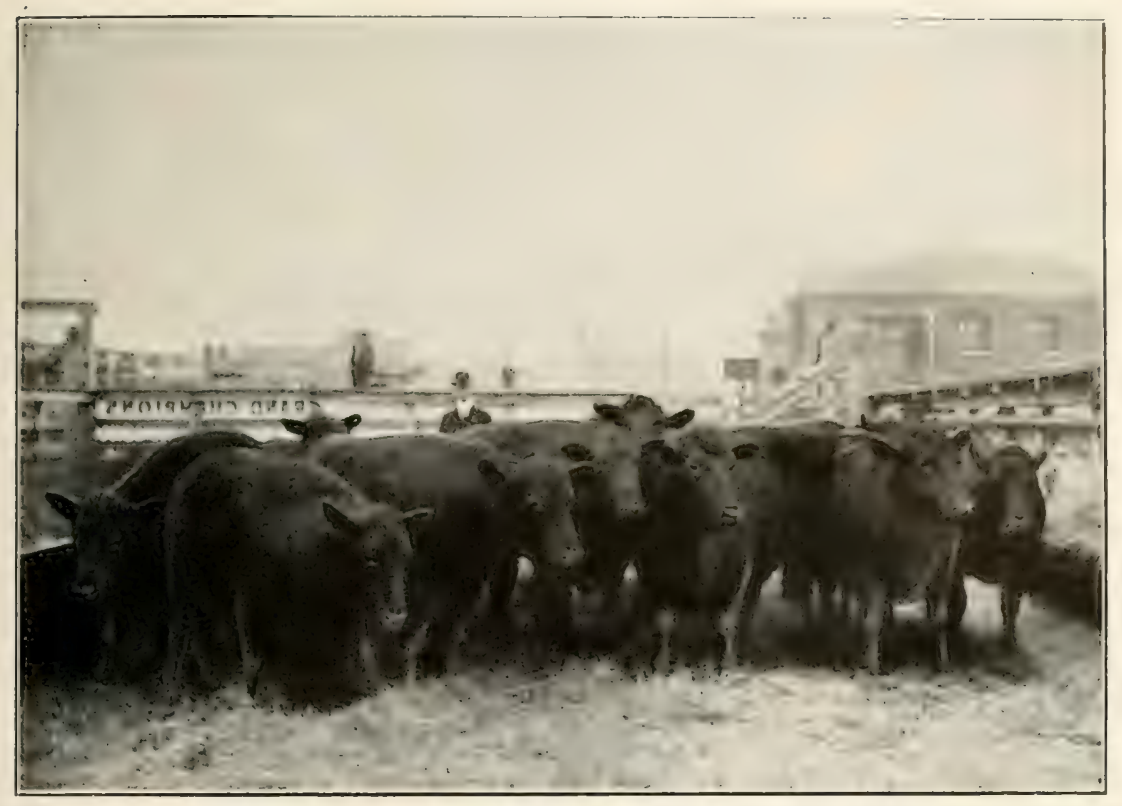

AN ABERDEEN-ANGLS PRODUCT OF TEXAS AND OKLAHOMA.

1910 Grand Champion Carload of Fat Steers over all Breeds at National Feeders and Breeders' Show, Fort Worth, Tcxas, Fed and Exhibited by H. B. Johnson

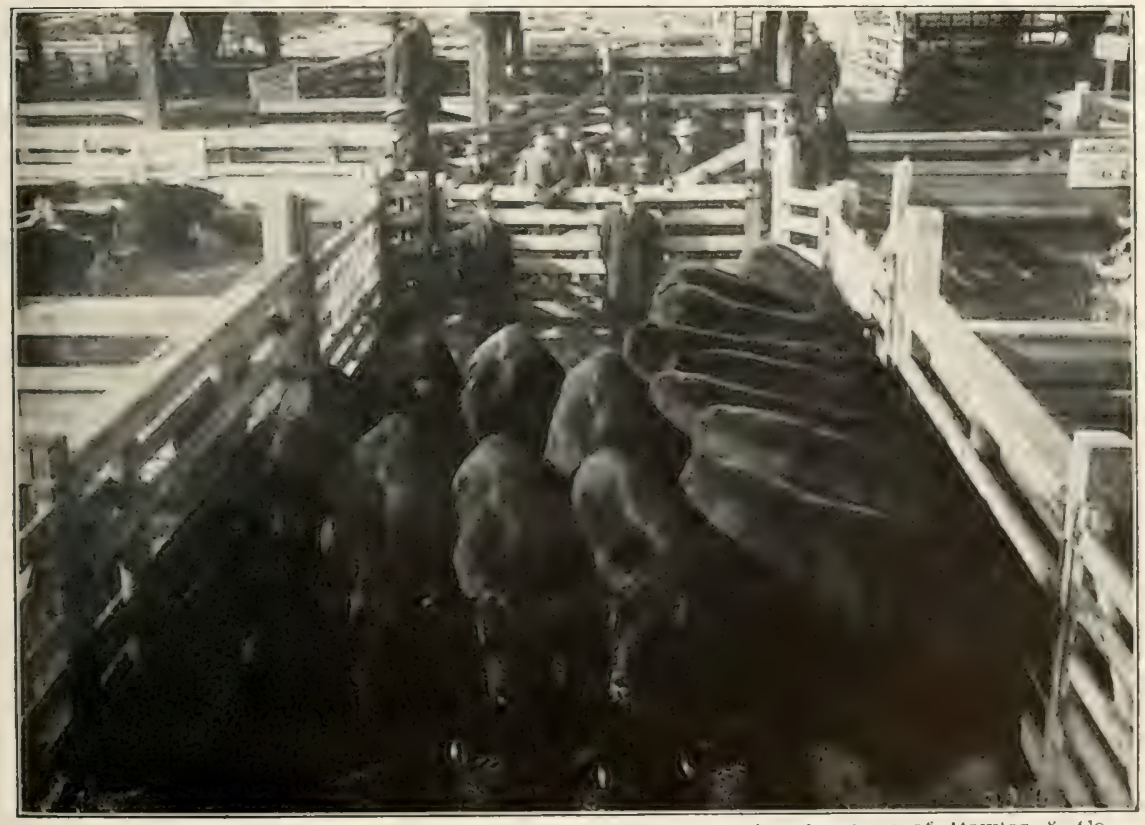

AN ABERDEEN-ANGUS PRODUCT OF MISSOURI.

Champion Carload of Yearling Fat Steers at 1908 International Live Stock Exposition. Fed and Exhibited by IV. C. White. 


\title{
Chicago International Live Stock Exposition Carcass Contest Awards 1900-1913
}

\author{
Competition Open to Steers, Spayed or Martin Heifers
}

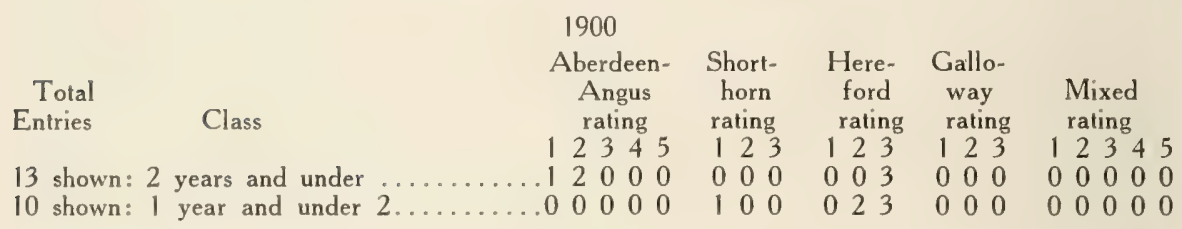

1901.

Total

Entries

Class

Aberdeen-

Angus

rating.

rating.

Short-
horn
rating.

Here-

Gallo-

33 shown: 2 years and under $3 \ldots \ldots \ldots \ldots 12300$

$12345 \quad 12345$

12345

13 shown: 1 year and under 2 .

00

$\begin{array}{lllllllllll}0 & 0 & 0 & 0 & 0 & 0 & 0 & 0 & 0 & 0\end{array}$

1902

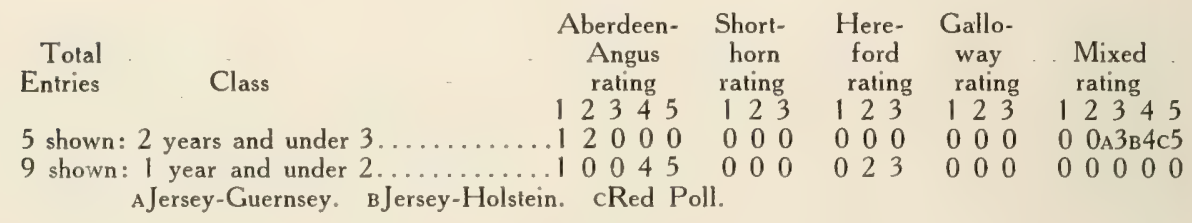

1903

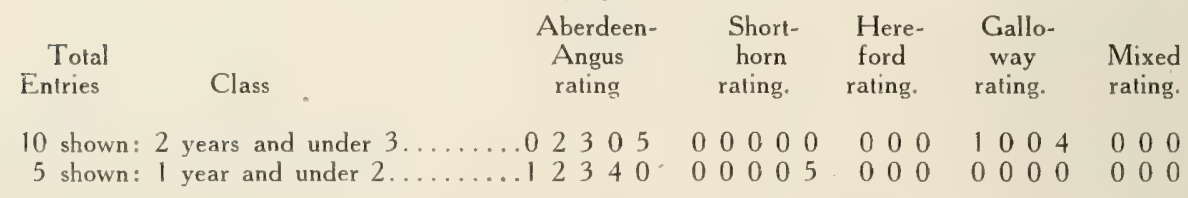

1904

Total

Entries.

Class

8 shown: 2 years and under $3 \ldots \ldots 123$

11 shown: 1 year and under $2 \ldots \ldots \ldots .003$

*Hereford-Holstein. ARed Poll. BShorthorn-Galloway.

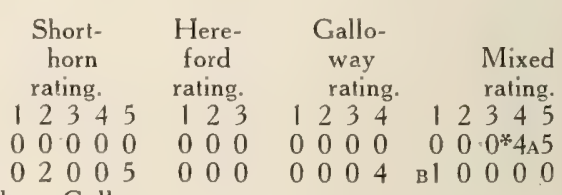

1905

Total

Entries

Class

\section{Aberdeen-}

Angus

rating.

1234

7 shown: 2 years and under $3 \ldots \ldots \ldots 10304$

6 shown: 1 year and under $2 \ldots \ldots .1000$

*Breed unknown. AShorthorn-Galloway.

\begin{tabular}{|c|}
\hline 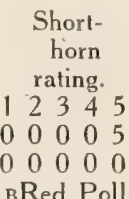 \\
\hline
\end{tabular}

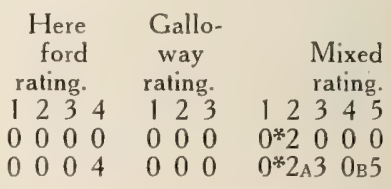


Total

1906

Entries

AberdeenAngus

Short-

Here- Gallo-

horn ford way Mixed rating

13 shown: 2 years and under 3 1 23345

14 shown: 1 year and under $2 \ldots \ldots \ldots .0000000$ AMixed. *Galloway-Shorthorn.

rating rating rating rating

$\begin{array}{lllllll}123 & 123 & 123 & 23\end{array}$

$\begin{array}{llllllllllllll}0 & 0 & 0 & 0 & 0 & 0 & 0 & 0 & 0 & 0 & 0 & 0 & 0 & 0\end{array}$

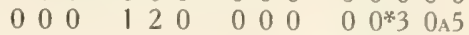

1907

Total

Entries.

Aberdeen-

Angus

Short-

horn

rating.

i5 shown: 2 years and under $3 \ldots \ldots \ldots \ldots 120 . \ldots 4$

123

$\begin{array}{lll}0 & 0 & 0\end{array}$

16 shown: 1 year and under $2 \ldots \ldots \ldots \ldots$ I 2040

000

*Polled Hereford. aRed Poll.

Here- Gallo-

ford way Mixed

rating. rating. rating.

$\begin{array}{lllllllll}1 & 2 & 3 & 4 & 5 & 1 & 2 & 3 & 2\end{array}$

$\begin{array}{lllllllllll}0 & 0 & 0 & 0 & 0 & 0 & 0 & 0 & 0 & 0 A 3\end{array}$

$\begin{array}{lllllllllll}0 & 0 & 0 & 0 & 5 & 0 & 0 & 0 & 0 & 0 * 3\end{array}$

1908

Total

Aberdeen- Short- Here- Gallo-

Entries. Class

Angus horn ford way Mixed

rating rating rating rating rating

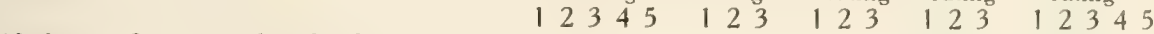

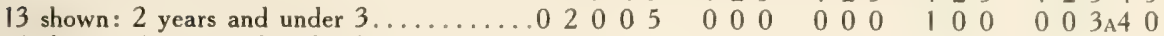

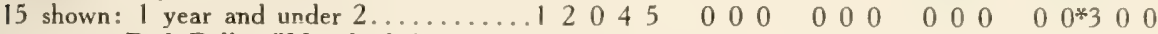

aRed Poll. *Hereford-Angus.

1909

Total
Entries.

7 shown: 2 years and under 3

Aberdeen-

Angus

rating

12345

Gallo-

Red

10300

way

rating

Poll

12345

rating

14 shown: 1 year and under 2

$\begin{array}{llllll}0 & 2 & 0 & 4 & 0\end{array}$

12345

14 shown: 1 year and under 2 .

\section{Total}

Entries. Class.

Aberdeen-
Angus
rating

1910

Short-

horn

6 shown: 2 years and under 3.100000

2345

$\begin{array}{lllll}0 & 0 & 3 & 0 & 5\end{array}$

$\begin{array}{lllll}0 & 0 & 0 & 0 & 0\end{array}$

$\begin{array}{lllll}0 & 0 & 0 & 0 & 5\end{array}$

$\begin{array}{lllll}0 & 0 & 0 & 0 & 0\end{array}$

11 shown: 1 year and under 2.1 20005

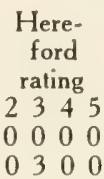

Gallo-

way

rating

12345

020000

$\begin{array}{lllll}0 & 0 & 0 & 0 & 0\end{array}$ aRed Poll. *Galloway-Shortlorn.

$\begin{array}{cc}\text { Cotal } & \begin{array}{c}\text { Aberdcen- } \\ \text { Angus } \\ \text { Entries. }\end{array} \\ \text { rating }\end{array}$

1911

Short- Here- Gallo-

horn ford way

rating rating rating

Mixed

$12345 \quad 12345 \quad 12345$

$\begin{array}{lllllllllllllll}0 & 0 & 0 & 4 & 0 & 0 & 2 & 0 & 0 & 0 & 0 & 0 & 0 & 0 & 0\end{array}$

$\begin{array}{llllllllllllll}0 & 0 & 0 & 0 & 0 & 0 & 0 & 0 & 0 & 0 & 2 & 0 & 0 & 0\end{array}$

Mixed rating

12345

$\begin{array}{llllll}0 & 0 & 0 & 4 \mathrm{~A} 0\end{array}$

$\begin{array}{llll}0 & 0 & 0 & 4 * 0\end{array}$

5 shown: 2 years and under 3.10000

10 shown: 1 year and under 2.103445 ARed Poll.

1912

Total

Entries.

Class.

Aberdeen-

Anerdeen
Angus

rating

I 2345

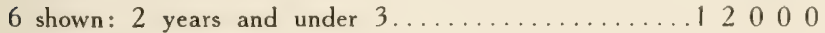

11 shown: 1 year and under $2 \ldots \ldots \ldots \ldots \ldots \ldots \ldots \ldots \ldots \ldots$

Shorthorn rating

12345

$\begin{array}{lllll}0 & 0 & 0 & 0 & 5\end{array}$

000000
Red Poll

rating

12345

$\begin{array}{llllll}0 & 0 & 3 & 4 & 0\end{array}$

00000

1913

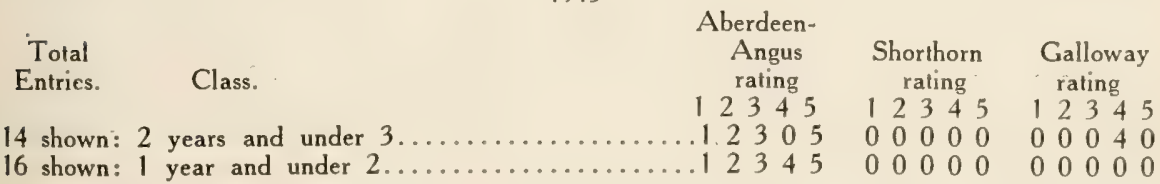




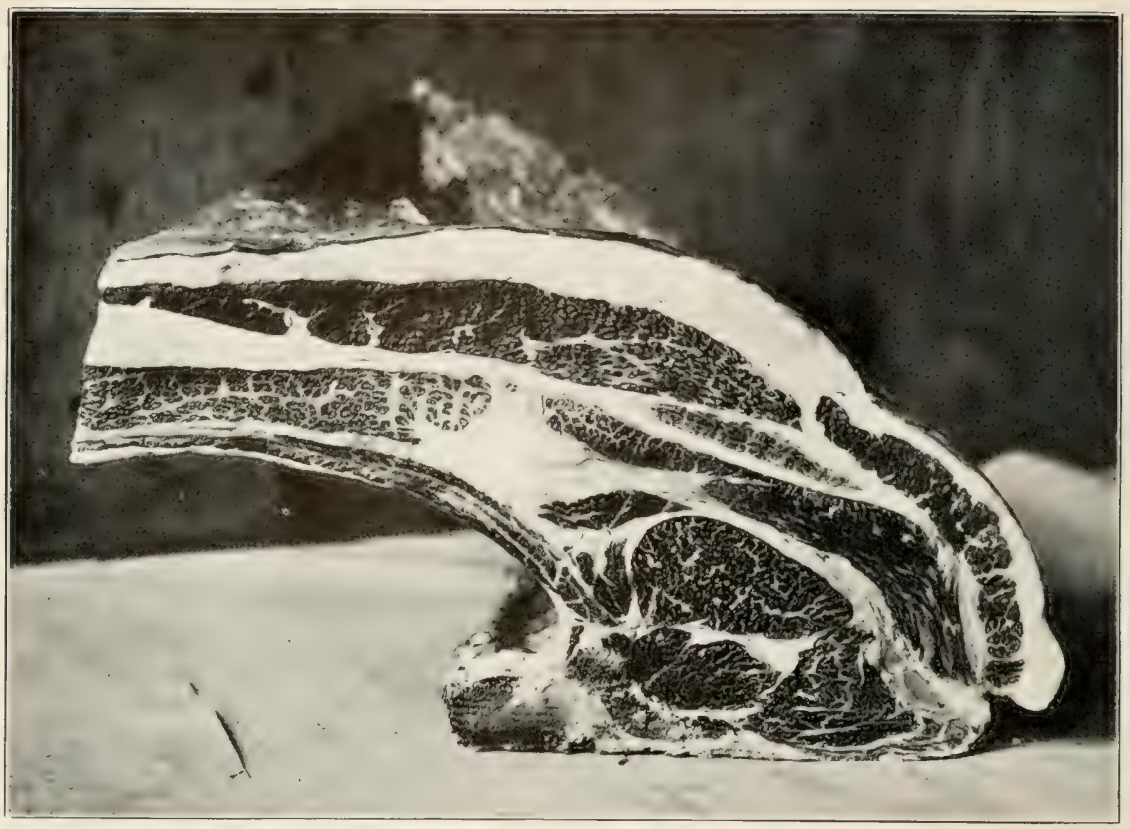

CHUCK OF ABERDEEN-ANGUS STEER EXILIO

Best Grand Champion Careass of any Breed that has been Exhibited at Internatiunal Live Stock Exposition.

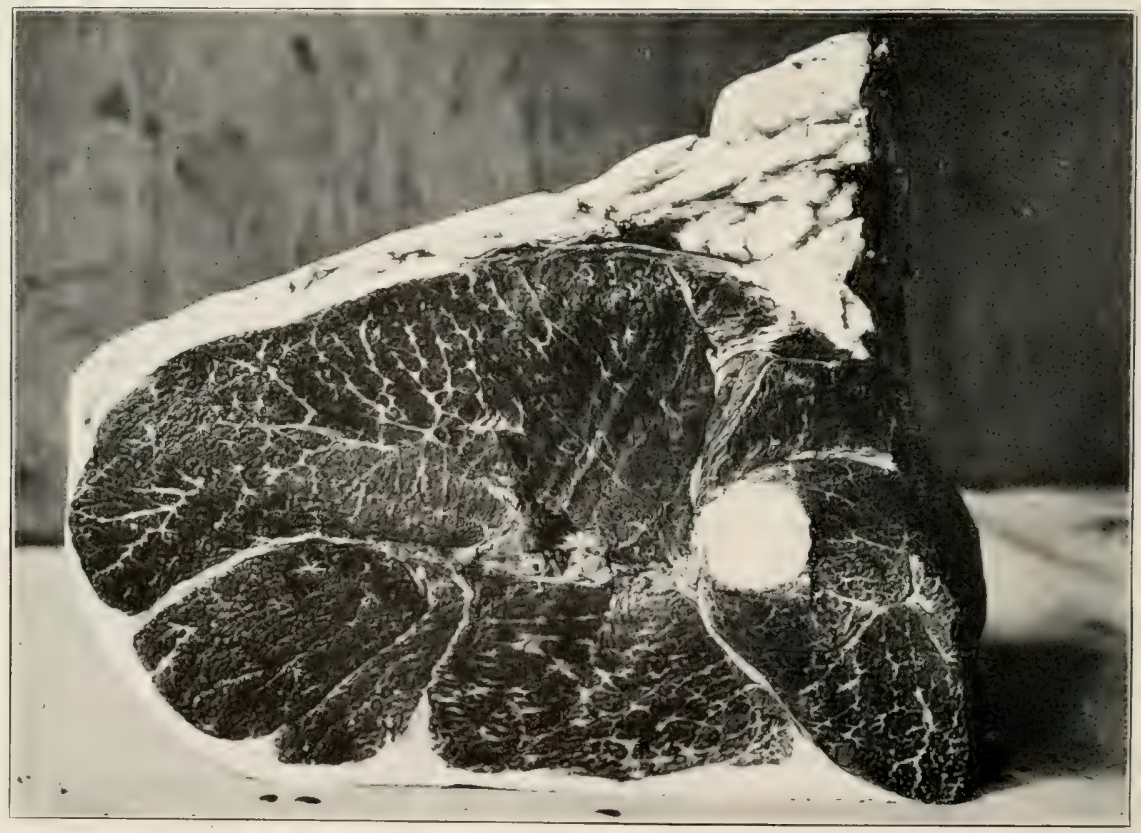

ROUND OF ABERDEEN-ANGUS STEER EXILIO.

Best Grand Champion Carcass of any Breed that has been Exhibited at International Jive Stock Exposition. 


\section{Grand Champion Carcass Awards, 1900-1913}

YEAR NAME

......... B. Bunker. .

... None

1901 Elm Park Lad.........Mich. Agricultural Col.... Third

1903 College Lad......... lowa State College........Fifth . . . . . Aberdeen-Angus

1904 Funk's Choice........ Funk Bros................. sot shown.... Grade Aberdeen-Angus

1905 College Lad.......... Iowa State College....... None.......Aberdeen-.-Angus

1906 Exilio............... . J. Off............ Fifth . . . . . . Aberdeen-Angus

1907 Squire Good........... L. Taggart.................... Grade Aberdeen-Angus

1908 Ben H........... D. Bradfute \& Son.......Fifth *.....Aberdeen-Angus

1909 La Preto............. University of Nebraska.... Third........Aberdeen-Angus

1910 Crown............. C. L. Taggart......... Third.......Aberdeen-Angus

1911 Model.............. Mart L. McCoy ..........First . . . ....Aberdeen-Angus

1912 Prince of View Point 5th. University of Nebraska.... First . . . . . Aberdeen-Angus

1913 Star of the North...... University of Minnesola... First ........Aberdeen-Angus

*A special class for carcass cattle.

\section{SUMMARY OF INTERNATIONAL LIVE STOCK EXPOSITION CARCASS CONTESTS, 1900-1913}

A review of the carcass competition of the past fourteen International Live Stock Expositions reveals the fact that Aberdeen-Angus cattle and their grades have won seventy-six prizes out of a possible one hundred and thirty-two. This is 58 per cent. of all money prizes offered. Aberdeen-Angus have also won thirteen out of the fourteen possible Grand Championships for dressed carcasses. Analysis of the awards covering the period of fourteen years further shows the degree in which Aberdeen-Angus and their grades excel all other breeds on the block. The seventy-six Aberdeen-Angus winners were composed of thirty-seven two-year-olds and thirty-nine yearlings. Thirty-seven were pure-breds, thirty-seven were grades and two were cross-bred Hereford-Aberdeen-Angus.

Further elaboration to establish the phenomena'. merits of the Aberdeen-Angus is not needed because a glance at the results of the carcass contests clearly demonstrates the superiority of the Aberdeen-Angus over all breeds. 


\title{
Aberdeen-Angus Cattle on the Range
}

\author{
By GEORGE FINDLAY, of the X. I. T. Ranches.
}

Aberdeen-Angus cattle are not without a record in the United States under grazing conditions as they have existed and exist now beyond the Missouri River. The breed has been tried out in more than one section and in every instance the performance was satisfactory in every respect to those whose money was involved. My own experience with these cattle has, however, been on what are popularly known as the X. I. T. Ranches in Texas, owned by the Capitol Syndicate.

Up to and including 1892 there were purchased for the X. I. T. runges, not far from 5,000 bulls, of which Aberdeen-Angus comprised not quite 14 per cent, Herefords about 50 per cent, and Shorthorns not quite 30 per cent. The small proportion of Aberdeen-Angus was due to the fact that until a few years previous it was a breed comparatively unknown in this country. Its numbers were inconsiderable and bull were hard to get, se!ling at much higher figures than those of any other breed. The owners and managers of the property were favorably disposed toward the breed and have continued in that attitude.

Originally the X. I. T. Ranch comprised 3,000,000 acres of land in the Panhandle of Texas. The cattle with which it was stocked came largely from the country tributary to the Texas \& Pacific Railway and were of better average quality than those common to the country. About the early nineties most of the bulls purchased were pure-breds, and after 1892, nothing but pure-breds were bought. The range, averaging about 200 miles north and south and 25 miles cast and west, and being all fenced and cross-fenced, offered good opportunities to test the three breeds under practically similar conditions and, after a few years, the pastures in which each was kept began to show the respective breed characteristics. Every year, by careful selection of breeding bulls and careful culling of undesirable females - undesirable owing to color or qua'ity-the herds rapidly assumed, to all appearances, the quality and character of pure-breds. When this experiment was begun the Shorthorn breed was well known by reputation in the Southwest. They had been tried previously in the section from which the foundation she stock had been purchased and their reputation at that time was, whether deserved or undeserved, that they were good cattle, but not sufficiently hardy for the climate of the Texas Panhandle. At that time Herefords were being introduced and had no prejudice to contend with, as few of the people there knew anything about them as ranging cattle. They were readily adopted by 
ranchmen seeking something to improve their herds and were extensively introduced into the Panhandle. The Aberdeen-Angus came in after the Herefords, but at this time they were few in number in the United States and it was impossible to secure them to the number required at prices range men could afford to pay. Herefords were being pushed by a coterie of breeders exultant over con flicts from which they had emerged with Shorthorn sponsors and were claiming "the earth and the fullness thereof" for their breed. As nothing succeeds like success, the Herefords soon became the dominant breed in the Panhandle. It was diligently published by interests antagonistic to the Aberdeen-Angus, and actuated either by ignorance or jealousy of the breed, that it was not suitable for range purposes, that the bulls would bunch together and stay away from the she catt?e, consequently they did not get the percentage of calves possible with bulls of other breeds. Allegation was also made that the stock they did get did not exhibit sufficient improvement and that they could not stand the heat of summer or the rigors of winter. Thus it will be seen that the Aberdeen-Angus came into that territory at a rather unpropitious time and had to fight against ignorance, prejudice and jealousy for its foothold there.

These sentiments were not lacking on the X. I. T. Ranch, but after the adoption of the three breeds each was given a fair trial and the result there demonstrated that no breed was better adapted to range conditions than the AberdeenAngus. They proved themselves prolific, hardy, good rustlers, early maturers and good sellers, the steers of this breed being usually the first to be sold off the range and invariably commanding a premium over the others.

With these results it is logical that as the land comprising the ranch was sold off, over 2,000,000 of the original 3,000,000 acres having now been disposed of to Northern farmers, necessitated selling cattle also, the owners decided to close out the other breeds and retain the Aberdeen-Angus herd. At the present time that range carries no breeding cattle of any other breed. This policy, shaped after probably better facilities for tcsting the breeds than have ever been afforded anywhere e'se, speaks more for the merits of Aberdeen-Angus cattle as a range breed than columns of theory and argument. Had the Aberdeen-Angus not given satisfactory results, they would have been the first to go. Actual results furnished convincing evidence that there is absolutely nothing to the claim that Aberdeen-Angus are poor breeders on the range. In 1889 there were practically the same number of cows in the A'amositas X. I. T. pasture, in which Aberdeen-Angus bulls were placed, as in the Minneosa pasture where Hereford bulls were used. In 1890 the calves branded in the Alamositas pasture numbered 3.064, those in the Minneosa pasture 2,688, and there were branded in the pasture in which black bulls were kept during the years immediately following a greater number of calves than in the other pastures.

Satisfactory results were also reached with Abcrdeen-Angus cattle in Montana, where they grazed on open range and among $X$. I. T. owners and man- 
agers there exists no doubt regarding the fecundity of black bulls. Mr. A. G. Boyce, manager of the Texas ranch, reporting on the best results, said: "The more I see of the black cattle the more I like them and think they are the cattle for this country."

It may be of interest to those seeking information regarding Aberdeen-Angus cattle on the range to know that we have always considered steers of this breedboth as feeders and beeves-the quickest and best sellers, and when time and conditions permit, we have always found it to our advantage to ship AberdeenAngus beeves by themselves, as there seemed to be a wider market for them at the stock yards and they have almost invariably realized better prices than the others. Probably 75 per cent of all the fat steers reaching market nowadays are without horns. Even on the ranges many dehorn their bulls. This dehorning practice is one of the strongest tributes to the value of the polled character of the Aberdeen-Angus that can be conceived.

\title{
Aberdeen-Angus Cattle in the Feed Lot
}

\author{
By A. W. Bragg of Tuscola, Ill.
}

My preference for black cattle in the beef making operation is the result of 40 years experience. The only complaint I have to make is the difficulty in securing them.

I do not wish to be put in the position of disparaging the other beef breeds but an Aberdeen-Angus steer is always equal to a feed lot performance possible with no other.

They are not only "good doers" but they are the most stretchy cattle I can get. They may be put on feed at any age with profitable results.

Take an Aberdeen-Angus calf at weaning time and he may be converted into a choice yearling, or, if market conditions necessitate it carried along another year with good results.

The Aberdeen-Angus never geis coarse or gobby. This accounts for its popularity at the market. I prefer them to any other breed in filling my feed lots. They are ideal in type, temperament, quality and gain-making capacity. 


\section{A Notable Market Event}

Market performance by Angus catt'e is an every-day occurrence. The salesman has merely to intimate to the buyer that he has a drove of "blackskins" to command attention. Dressing sheet results and quality on the hooks affords the exp'anation.

But on the market session of July 23 at Chicago an incident occurred that deserves mention in trade annals. On that date a drove of 73 head of 957 -pound grade berdeen-Angus yearlings went over the scales at $\$ 9.95$ per cwt., which was only 5 cents below the season's limit on aged long-fed cattle and 20 cents above the best previous performance by the babies. These were not pure-bred cattle, eligible to registry and picked out of the calf crop with the object of doing a pyrotechnical market stunt, but just a herd of every-day grade Aberdeen-Angus such as any feeder has access to. They were gathered in Howard County, Mo., in the fall of 1913 at weaning time by officials of the Illinois Experiment Station for the purpose of a test of the comparative merits of corn silage and alfalfa in making economical gains. To give this experiment value the use of commercial catt'e was imperative.

The drove dressed 63.2 per cent hot and the product went to high class eastern trade with which Aberdeen-Angus beef is always popular. Some of these calves more than doubled weight in the feed lot. They were put in Nov. 22, 1913, and were fed 238 days. For experimental purposes they were fed in seven lots and the data resulting is not uninteresting:

$\begin{array}{lccccccc} & \text { Lot } & \text { Lot } & \text { Lot } & \text { Lot } & \text { Lot } & \text { Lot } & \text { Lot } \\ & 1 & 2 & 3 & 4 & 5 & 6 & 7 \\ \text { Initial weight per steer..... } & 497.5 & 490.0 & 495.5 & 489.5 & 497.5 & 499.0 & 485.0 \\ \text { Final weight per steer...... } 1005.0 & 975.5 & 1003.0 & 997.5 & 987.5 & 1015.5 & 952.2-9 \\ \text { Total gain per steers...... } 507.5 & 485.5 & 507.5 & 508.0 & 490.0 & 516.5 & 467.2-9 \\ \text { Average daily gain per steer } & 2.13 & 2.04 & 2.13 & 2.13 & 2.06 & 2.17 & 1.97 \\ \text { Pork produced per steer.... } & 32.8 & 47.7 & 46.1 & 45.4 & 42.0 & 29.3 & 41.4 \\ \text { Cost of gains per cwt..... } \$ 7.96 & \$ 8.94 & \$ 8.33 & \$ 8.66 & \$ 8.20 & \$ 9.07 & \$ 9.14 \\ \text { Necessary selling price..... } & \$ 8.40 & \$ 8.90 & \$ 8.59 & \$ 8.75 & \$ 8.53 & \$ 8.87 & \$ 8.99 \\ \text { Profit per steer (pork in- } & & & & & & & \\ \text { cluded) ............. \$12.70 } & \$ 9.27 & \$ 12.91 & \$ 12.13 & \$ 12.03 & \$ 8.86 & \$ 7.41\end{array}$

The figures afford a demonstration of the adaptability of the Aberdeen-Angus calf to put on weight at a profit even during a period of high-priced feed, not to speak of the premium to which they are sligible at the market by virtue of superior quality and salability of the beef and satisfactory dressing percentages. 


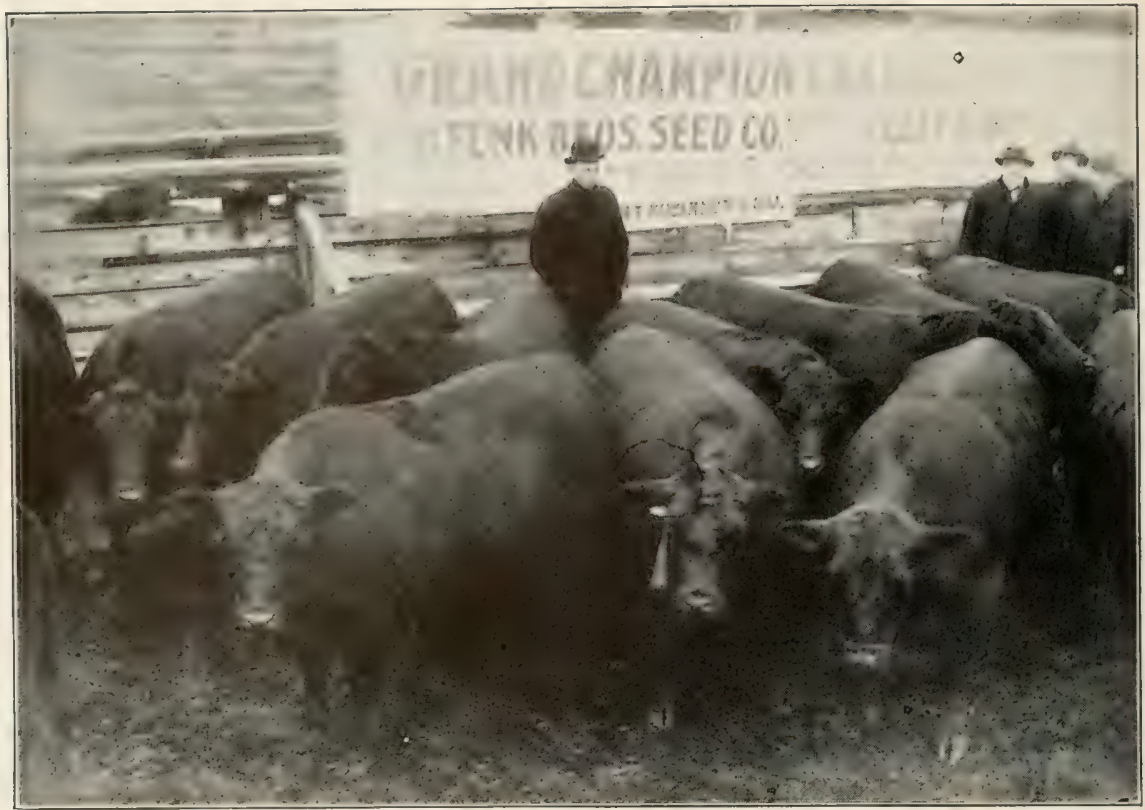

AN ABERDEEN-ANGUS PRODUCT OF ILLINOIS.

Grand Champion Carload Fat Steers over all Breeds, 1906 International Live Stock Exposition. Fed and Exhibited by Funk Bros., and Sold at 17e Per Pound by Clay, Robinson \& Co., which is the Record Price for a Carload at Chicago.

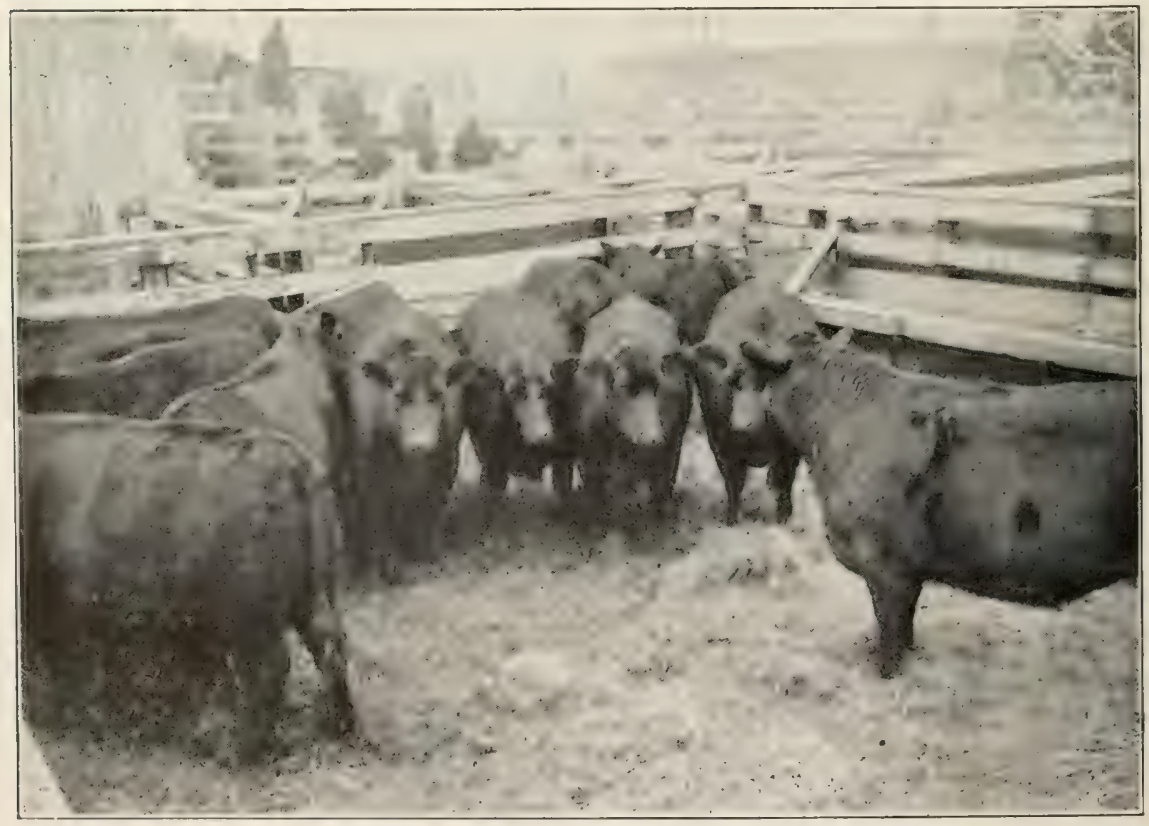

AN ABERDEEN-ANGUS PRODLCT OF IOWA.

Grand Champion Carload Fat Steers at 1906 American Royal Live Stock Show, Kansas ('ity, Mo. Fed and Exhibited by the late Claus Krambeck. 


\title{
South St. Joseph Inter-State Live Stock Show Awards
}

Held at South St. Joseph. Mo. (This Show was instituted in 1906.)

GRADES AND CROSS-BREDS.

Competilion limited to Sicers. Spayed or Martin Heifers.

\section{GRAND CHAMPION AWARDS ON SINGLE STEERS AND FAT CARLOAD LOTS.}

Grand Champion Single Steer BREED

1906 Aberdeen-Angus

1907 Shorthorn

1908 Aberdeen-Angus

1909 Shorthorn

1909 Reserve, Aberdeen-Angus

1910 Shorthorn

1910 Reserve, Aberdeen-Angus

1911 Aberdecn-Angus
Grand Champion fat Car Lot BREED

1908 Aberdeen-Angus

1909 Hereford

1909 Reserve, Aberdeen-Angus

1910 Hereford

1911 Hereford

\section{Inter-State Fair, Sioux City, lowa}

\section{GRAND CHAMPION AWARDS ON SINGLE STEER AND STEER HERD,} 1905-1912.

(Awards, if any, of 1903 and 1904 could not be obtained.)

Grand Champion Single Steer BREED

1905 Aberdeen-Angus

1906 Aberdeen-Angus

1907 Shorthorn

1908 Aberdeen-Angus

1909 Aberdeen-Angus

1910 Aberdeen-Angus

1911 Shorthorn

1912 Hereford

1909 Grand Champion Fat Carload Lot, Aberdeen-Angus.
Cirand Champion Steer Herd BREED

Aberdeen-Angus

Aberdeen-Angus

Shorthorn

Aberdeen-Angus

Aberdeen-Angus

Aberdeen-Angus

Hereford

\section{lowa State Fairs, 1895-1905}

\author{
GRAND CHAMPION BEEF HERD AWARDS. \\ BREED

\section{NAME OF EXHIBITOR}

1895 Aberdeen-Angus

1896

1897

1898

1899

1900

1901

1902

1903

1904

1905 No fair held.

W. A. McHenry, Denison, Iowa

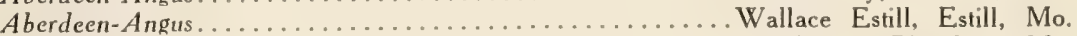
Hereford. ....................... Jas. A. Funkhouser, Plattsburg, Mo.

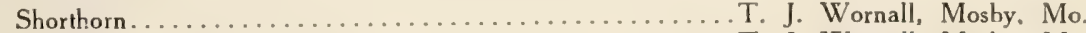

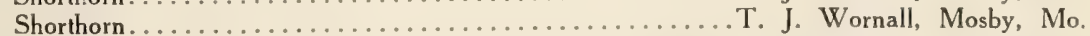

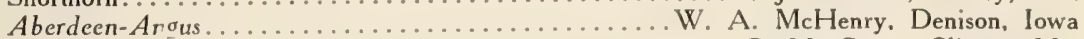
Shorthorn. .............................. Casey, Clinton, Mo. Aberdeen-Angus. ..................... ... Gardner, Blandinsville, Ill. Aberdeen-Anous.......................... J. Martin, Jefferson, Iowa

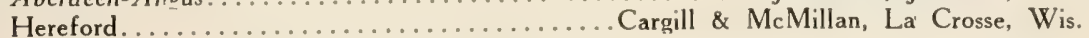




\section{American Royal Live StockShow, Kansas City,Mo.}

1906 Grand Champion Fat Carload, Aberdeen-Angus.

1910 Grand Champion Fat Carload, Hereford.

1910 Reserve Grand Champion Fat Carload, Aberdeen-Angus.

\section{Western Stock Show, Denver, Colo.}

GRAND CHAMPION AWARDS ON SINGLE STEER AND FAT CARLOAD LOTS, 1906-1914.

Grand Champion Single Steer BREED

1906 Shorthorn

1907 Aberdecn-Angus

1908 Hereford

1909 Aberdeen-Angus

1910 Shorthorn

1911 Hereford

1909 Grand Champion Steer Herd, Aberdeen-Angus.

1910 Grand Champion Carload of Feeders, Aberdeen-Angus.

1911 Grand Champion Carload of Feeders, Herefords.

1911 Reserve Grand Champion Carload of Feeders, Aberdcen-Angus.

1912 Grand Champion Carload of Feeders, Herefords.

1913 Herefords.

1914 Aberdeen-Angus.

\section{Fort Worth National Show, Fort Worth, Tex.}

\section{GRAND CHAMPION AWARDS ON SINGLE STEER AND FAT CARLOAD} LOTS, 1905-1913.

(No Aberdeen-Angus shown prior 1o 1905.)

Grand Champion Single SteEr

1905 Hereford

1906 Hereford

1907 Shorthorn

1908 Hereford

1909 Shorthorn

1910 Aberdeen-Angus

1911 Hereford

1912 Hereford

1913 Hereford

\section{Shorthorn}

Aberdeen-Anguis

Aberdecn-Angus

He elo:d

Aberdeen-Angus

Aberdeen-Angus

Aberdeen-Angus

Reserve, Aberdeen-Angus

Hereford

Rese:ve, Aberdeen-Angus

Aberdeen-Angus

\section{Brandon Winter Stock Show}

\section{Brandon, Manitoba, Canada}

Grand Champion Single Steer

1910 Aberdeen-Angus

1911 Aberdeen-Angus

1912. Aberdeen-Angus

1913 Aberdeen-Angus

1914 Aberdeen-Angus
Grand Champion Steer Herd

Aberdeen-Angus

Aberdeen-Angus

Aberdeen-Angus

Aberdeen-Angus

Aberdeen-Angus

At the greatest Canadian Fat Stock Show held in Eastern Canada, at Guelph, Ontario, an Aberdeen-Angus steer won Grand Championship over all breeds in 1908 and Grand Champion Steer in 1912 and 1913. An Aberdeen-Angus also won Grand Championship over all breeds at Toronto National Fat Stock Show in 1913. 


\section{Pure Bred Cattle Sales}

COMPARATIVE AVERAGES OF AMERICAN PUBLIC SALE PRICES FOR TEN YEARS, AS REPORTED BY “BREEDERS' GAZETTE," 1904-1913.

No. of
Sales

NAME OF BREED

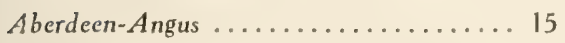

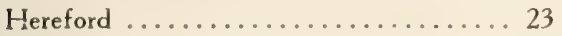

Shorthorn ................. 48

Gialloway ....................... 1

Polled Durham ................... 3

Red Poll

\section{NAME OF BREED}

Aberdeen-Angus ............... 13

Hereford ..................... 19

Shorthorn ................. 53

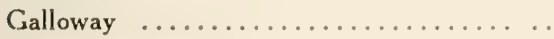

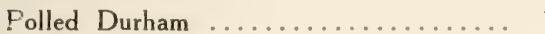

Red Poll

NAME OF BREED

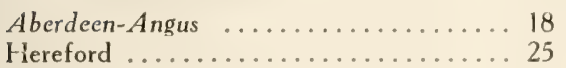

Shorthorn ..................... 78

Galloway ................. 2

Folled Durham .................. 2

Red Poll

1913

NAME OF BREED

1 berdeen-Angus ............... 18

Hereford ..................... 29

Shorthorn .................. 84

Calloway ................... 3

Polled Durham .................. 3

Red Poll .................... 3

NAME OF BREED

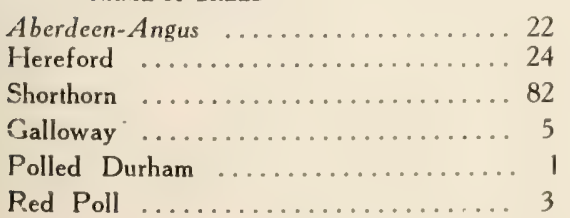

No.

Sold

797

1,311

2,175

24

106

...

1911

723

1,203

2,258

...

42

...

1909

935

1.398

3,309

69

79

35

1907

1,119

1,358

3,608

123

106

7

1905

Av. No. of No.

Sales Sold

Av.

Price

$\begin{array}{rrrr}\$ 171.95 & 12 & 627 & \$ 138.95 \\ 259.30 & 15 & 957 & 180.40 \\ 220.35 & 45 & 1,882 & 177.40 \\ 162.00 & \ldots & \ldots & \ldots \ldots \\ 131.70 & 2 & 83 & 132.85 \\ \ldots & 1 & 30 & 107.25\end{array}$

1910

\section{$\$ 143.60$}

160.50

162.50

19

995

$\$ 167.35$

$1.214 \quad 146.20$

1,999

187.50

67

83.30

140.60

74

115.00

41

185.00

1908

$\$ 189.00$
127.05
159.00
128.05
129.45
97.80

955

$\$ 165.10$

116.15

$2.689 \quad 146.50$

$136 \quad 84.50$

$244 \quad 124.50$

$3 \quad 50.00$

1906

$\$ 134.75$
123.70
160.15
139.05
130.35
83.65

1,259

$\$ 154.90$

121.15

144.90

108.85

49

143.40

$30 \quad 121.00$

1904

$\begin{array}{crrrr}1,034 & \$ 130.35 & 21 & 932 & \$ 132.80 \\ 1,179 & 115.35 & 28 & 1,481 & 117.10 \\ 3,512 & 139.75 & 65 & 2,755 & 101.25 \\ 190 & 103.85 & 3 & 133 & 143.55 \\ 34 & 231.75 & 7 & 286 & 100.00 \\ 94 & 109.80 & 1 & 48 & 70.00\end{array}$




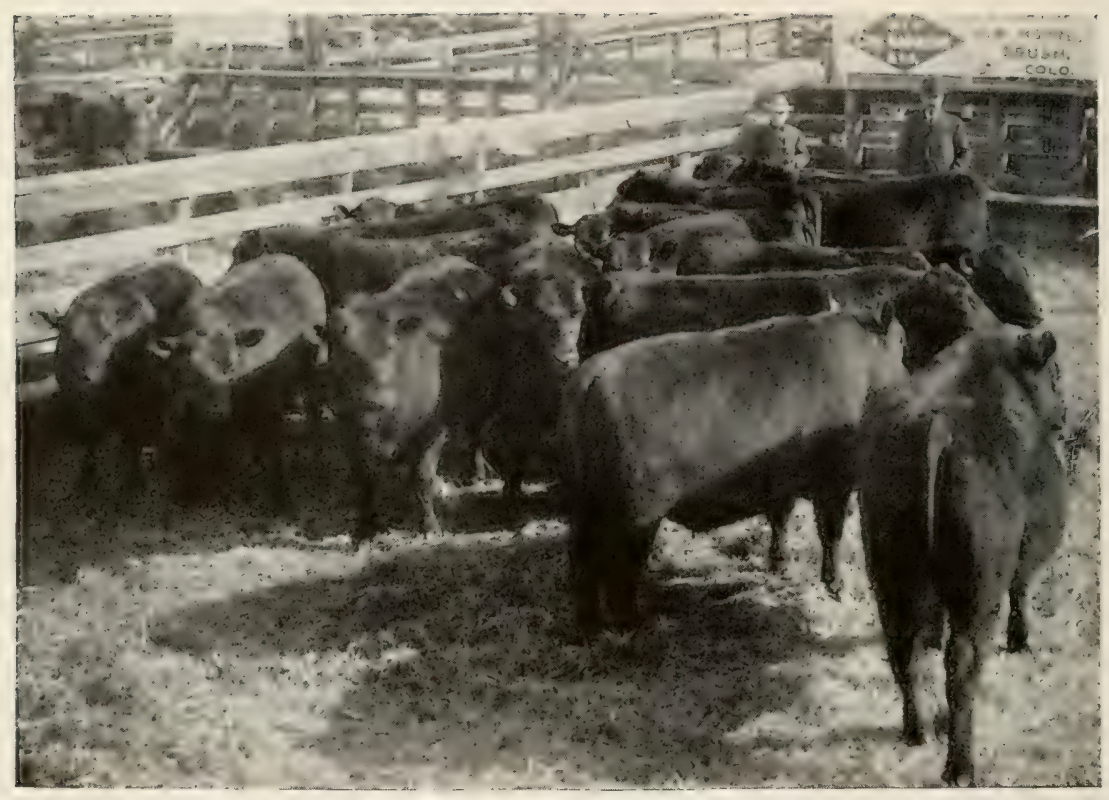

AN ABERDEEN-ANGUS PRODUCT OF COLORADO.

Grand Champion Carload Fat Steers over all Breeds at 1908 Western Stock Show, Denver, Colo. Fed and Exhibited by $H$. W. Moore.

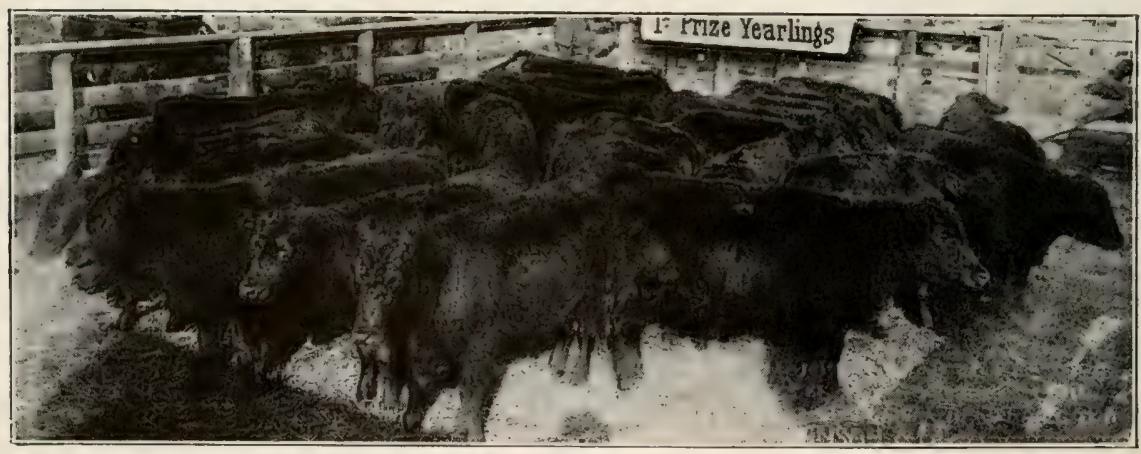

AN ABERDEEN-ANGUS PRODUCT OF COLORADO.

Grand Champion Carload of Feeders over all Breeds at 1910 Western Stock Show, Denver, Colo.. Bred and Exhibited by George F. Lucore 


\section{Why the Aberdeen-Angus Cattle are Popular with Breeders, Feeders, Packers, Butchers and Consumers}

In an exhaustive review of the beef houses of the leading packers of Chicago, covering a period of several years during which time the packers, wholesale beef market managers, and salesmen were systematically and minutely consulted, the following lerse and unanimous opinions were obtained:

The form and quality the Aberdeen-Angus breeders of Great Britain and America have succeeded in perfecting, in a large degree through the educational medium of such shows as International Live Stock Exposition, Chicago, and Smithfield Show, London, England, in their breed in a comparatively short time, have been instrumental in greatly raising the average standard of beef.

Since the systematic use of pure blood has been established in this country, a period which is scarcely worthy of consideration beyond the dates which the Aberdeen-Angus and the packing industry were substantially introduced, the quality or grade of beef animals that have come to market has shown a distinct and constant improvement.

The breeder, packer, butcher and consumer are getting closer together as time advances to the mutual benefit of everyone concernd in production and consumption of beef.

The Aberdeen-Angus bullock is a prime favorite with the packer's buyer because he possesses the desired form, being as a rule exceptionally well developed in loin, crops, back, thigh, twist and rump, the parts from which the high-priced cuts are secured.

The wholesale market popularity of the Aberdeen-Angus has been brought about by the sheer merit the carcasses of well-bred animals of the breed have invariably exhibited on the hooks. The packers' sa'esmen like the AberdeenAngus because their carcasses fill the eye of the butchers and therefore command ready sale at top prices.

The inherent early maturing characteristics of the Aberdeen-Angus bullock bring him to market very young at a time when the quality of beef is at its best to cut up economically for the butcher's trade and satisfy the most fastidious consumer. The Aberdeen-Angus invariably hangs up a well proportioned caicass on the hooks which shows a large proporticn of lean meat, with just enough fat interspersed to give proper flavor and juiciness. The Aberdeen-Angus carcass is invariably characterized by compactness, smosthness and fireness of bone, attributes which are constantly sought by the butchers. The Aberdeen-Angus kvilock matures or ripens evenly ai any age and is always free from exterior gobs of fat, which is known in beef house circles as "soap stock."

We predict an unprecedented future for the Aberdeen-Angus bullock, even greater than he has enjoyed in the past, which everyone is familiar with, because 
he matures at the right age or any age and produces a quality of beef that always finds a ready outlet at top prices. Although the quality of beef has been greatly improved by the systematic efforts of breeders, packers and butchers, it is nevertheless true there is and always will be "plenty of room at the top" for improvement.

The demand for high class beef such as the Aberdeen-Angus bullock produces can never be overdone, thus it behooves every farmer and producer of beef to raise or produce Aberdeen-Angus bullocks or animals that possess their form and quality, which are considered the most economical and remunerative for the breeder, packer, butcher and consumer.

\section{The Aberdeen-Angus Leads All Other Breeds in Point of Early Maturity}

Much has been written pro and con about the merits of the leading beef breeds on the point of early maturity. The zealous advocates of their respective breed have claimed everything and made assertions that could never be substantiated because of the lack of conclusive facts.

The promoting policy of the American Aberdeen-Angus Breeders' Association has been and is at present to obtain all the actual results of the leading Fairs, Shows and Expositions of the country, especially the results of the leading Fat Stock Shows of Great Britain and America as well as the results of the investigators in all research work pertaining to the beef breeds of cattle.

The conclusions of Jas. A. B. W'atson and Ernest Harrison bear so accurately on the point of maturity that every Aberdeen-Angus breeder should fortify himself with, and spread among his neighbors, the following significant facts:

"In comparing the rate of growth in the different breeds the Aberdeen Angus attains its full weight more rapidly than does the Shorthorn or Hereford. This is true of both sexes."

The above statement and proof of the superior early maturing merits of the Aberdeen-Angus breed were obtained after an exhaustive and thorough investigation.

The practical and scientific significance of the above conc'usion should be the means of enlightening breeders and feeders of beef cattle throughout the country, because Professors Watson and Harrison have spent their entire lives in practical and scientific study of farm animals.

They received their degrees as Bachelors of Agriculture at Edinburgh University, Great Britain, took additional work at the leading universities in Germany, and later finished at the Iowa State College, where they received degrees as Masters of Agriculture. They are now holding responsible positions in agri- 
cultural colleges in Great Britain, and because of their unusual training and familiarity with live stock in Europe as well as in America, their conclusions are unusually valuable.

The results of Professors Watson and Harrison corroborate the unanimous opinions and statements of the experienced beef buyers and salesmen of such packing firms as Armour \& Co., Swift \& Co., Morris \& Co., Cudahy \& Co., Pfaelzer \& Co., etc. The leading packers are unable to allow their buyers and salesmen to give out signed statements concerning the comparative merits of the leading beef breeds. If it were possible to secure signed statements of the experiences of beef salesmen and buyers the degree in which Aberdeen-Angus lead and excel their rivals in point of early maturity could be more forcibly demonstrated.

The popularity of Aberdeen-Angus as baby beef is chiefly due to the inherent early maturing qualities of the breed.

The attractiveness of the Aberdeen-Angus baby beef carcass on the packers' beef house hooks and the economical and profitable manner the carcass cuts for the butcher has made the Aberdeen-Angus baby beef extremely popular.

The size and quality of the Aberdeen-Angus bu!lock makes him the most popular beef animal and it seems reasonable to predict the Aberdeen-Angus will long continue to lead all other breeds in point of early maturity and in the popularity the breed has gained among packers and butchers as the most perfect beef animal that the world has yet produced. 


\section{Progressive Agriculture Extracts from "The Country Gentleman," May 2, 1914}

George M. Rommel, Chief of the Federal Division of Animal Husbandry, Washington, D. C., has been heralding the possibilities of the South as a beef producer for many years, and while the work of the Department of Agriculture, especially Bradford Knapp's Division of Farmers' Co-operative Demonstration Work, has borne much fruit the past few years, the greatest strides in the cattle industry have been made the past year. A realization of the possibilities of the South as a beef producer has been in evidence for many months in a measure greater than the most optimistic anticipated.

Chief Rommel, when recently interviewed by "The Country Gentleman," stated as follows:

"The South is the coming catt'e country. The Scuth is full of native scrub cows that are good breeders. The tick is being annihilated. The pasture grasses are rich and I have produced beef in that country at a cost of less than five cents a pound. What the South needs is better breeding stock and more of it."

Then Mr. Rommel, presenting his idea of facilitating the pure-bred cattle industry in the South to a representative of one of the beef breeds of cattle, said:

"You get your breeders' association to hold sales of pure-bred breeding stock in the South and you'll sell every good animal you offer. The South wants these animals and it is deluging us with inquiries as to where they can be had. Will your association back up the soundness, breeding and genera! quality of any breeding stock it would offer at such sales? If so, then you and I can talk business."

"Of course," replied the man. "Our association will stand behind the representation made by any and all of its members."

"That won't do," declared Mr. Rommel emphatically. "If your association and the Department of Agriculture are going to co-operate you've got to do better. You've got to guarantee every animal offered-the association as a body must make this guarantee and stand responsib'e."

"Can't do that-never have done it."

Mr. Rommel proposed this altogether new and revolutionary scheme to other breeders and got the same answer. He kept going, however, and at last an officer of the American Aberdeen-Angus Association jumped at the chance.

But the enthusiasm didn't enthuse the individual members of the association as it did the officers. Who ever heard of an association holding sales in the South, the land of scrubby cattle and the cattle tick? It would be a waste of money to attempt it! 
So the Association did this: It took the money right out of its own treasury and bought a good herd of top-notch breeders. It sent those breeders to Montgomery, Alabama, and tried the experiment.

In the mean time Mr. Rommel had explained the situation to Bradford Knapp, in charge of Farmers' Co-operation Demonstration Work in the South, and had asked him to have his demonstration agents advertise the sale to all the farmers.

The result was that when the sale was held every farmer who wanted good blood for his beef herds was there to outbid his neighbor. Every farmer rested assured that he was getting the worth of every dol'ar he spent. Here was a breeders' association guaranteeing every animal offered; here was the Department of Agriculture advertising the occasion and doing everything to make it a success.

The association offered thirty-six head at this sale in Alabama, and thirty-six head were sold at topnotch prices; and before this article appears in print the American Aberdeen-Angus Association will have held another sale in Memphis, Tennessee, conducted under identically the same co-operation arrangement with the department.

"The best part of it all is that the Aberdeen-Angus Association has set a precedent," said Mr. Rommel. "It is the first time that an association has pledged its own name in connection with such a sale, and it is the first time that an association has gone down in its official pocket to furnish money for purchasing the animals offered.

"I can see a great deal more in these sales at Montgomery and Memphis than satisfied purchasers, satisfied sellers and better stock for the South upon which to build its cattle industry. I can see the beginning of the day when breeders' associations will co-operate with the Department of Agriculture in all lines of its work. And when that day comes the South and every other section of the country will be on a bedrock foundation so far as the livestock industry is concerned.

"I look for the other breeders' associations to fall into line as soon as the success of this initial experiment has become well known. Why shouldn't the Jersey breeders, the Shorthorn breeders, the Hereford breeders, the Percheron breeders, meet the department half way? We advocate better stock and more of it; we have the machinery to advise would-be purchasers as to where the stock we advocate can be had. All we want is that associations and not individuals stand sponsor for every animal sold, and they will find that the department can practice co-operation as well as preach it."

That this move will open new fie'ds to the established breeders' associations and will prove a blessing to the men in the South who are beginning to realize the value of their lands and their scrub cows, is not debatable. It is self-evident.

A year ago Mr. Rommel sounded a warning against the wholesale shipments 
of scrub cows from the South to the West for breeding purposes. He begged the original owners of these cows, which were being sold for from fifteen to twenty dollars a head, to hold on to them.

"It isn't the initial value of the cow that you must consider," he argued. "You are depleting the breeding stock of this section of the country and you will never be able to get it back. Your scrub cows are invaluable as breeders.

"In this country of natural and abundant pasturage you can breed these cows to pure-bred bulls and produce calves that at twelve or thirteen months of age will weigh 400 pounds more than the mothers.

"In this natural grass belt you can produce feeders cheaper than in any section of the United States. You can produce them at a cost of less than five cents a pound. In our feeding experiments in Alabama we have produced them at a cost of three cents!" 


\section{Recapitulation of American Fat Stock Shows}

A comprehensive review of the foregoing pages gives a concise, accurate survey of the beef cattle industry in general and the resu'ts and progress of the leading recognized pure-bred beef breeds in particular, in America. A careful study of the results of American Shows for the fourteen years should convince every cattleman that the Aberdeen-Angus breed is justly entitled to the honor of being termed the Premier Beef Breed.

With the axioms set forth in the foregoing tables supplemented by the unbiased opinions of such able authorities on the various phases of the cattle industry as James E. Poole and George Findlay, the editor fee's it is useless to burden these pages with more elaboration, because the field has been covered thoroughly, the merits of all the rival beef breeds justly considered and the supremacy of the Aberdeen-Angus accurately and sufficiently established.

The average market top prices established in the past by Aberdeen-Angus at International and other leading Shows clearly and very forcibly demonstrate the degree in which the doddie leads all other breeds. The Aberdeen-Angus hold the record for top prices by a margin of 81 cents per cwt. more than the Herefords and 66 cents per cwt. more than the Shorthorns, covering a period of fourteen years.

This is an age of progress and it can be truly said of the Aberdeen-Angus breed that it is keeping pace with the rapid development of agriculture. The sphere of Aberdeen-Angus cattle operations has been greatly enlarged the past few years. In addition to the unequaled laurels the breed has won at International Live Stock Exposition, Chicago, and its growth in popularity in the leading cornbelt states, the breed has conclusively demonstrated its ability to adapt itself to every condition that has been found in the various cattle districts of America.

The substantial manner in which the breed has popularized and fortified itself in the most fertile cornbelt states is borne out by the records of the AberdeenAngus Association and the daily receipts of the leading markets. The dissemination of the breed and its rapid growth on the ranges of the North, West and Southwest have been forcibly illustrated to the public by the achievements of the breed at Winter Fair, Brandon, Manitoba, Canada; Western Stock Show, Denver, Colorado; and National Breeders' and Feeders' Show, Fort Worth, Texas. Since the Aberdeen-Angus breed has bcen exhibited in the West and range country, it has been greatly in the minority; nevertheless, it has won the Single Fat Steer, Fat Carload Lot, Feeder Carload Lot and Carcass, etc., championship honors a greater number of times than any other breed. This is a feat worthy of careful consideration by range men, because these Shows have been conducted along practica! lines, thus their results are entirely practical. 
This is not only an age of progress in this country, but of expansion that has scarcely ever been approached during the history of any other country. While there has been great activity in nearly every kind of agriculture there has, naturally, been lack of enthusiasm in the pure-bred cattle industry until recently. The pronounced shortage of beef cattle reached its climax during the past year and at present farmers and breeders in every part of the country have fe't the stimulus of necessity and are now clamoring for pure-bred cattle with a vengeance that has made the pure-bred trade the best that has ever existed in America.

The universal superior merits of the Aberdeen-Angus breed give it a coveted position of prestige, thus it behooves every Aberdeen-Angus advocate at this particular time to lend his enthusiastic efforts in order that the doddie may accomplish greater achievements.

The cornbelt states have been liberal importers from Great Britain since the introduction of the breed to this country. During recent years the cornbelt has been an exporter to Canada and during the past year more Aberdeen-Angus cattle have been shipped to southern states from the cornbelt than during the entire history of the breed in America.

The co-operation of the United States Government has educated the southern farmer to a point where he is now fully aware of the vastness of the southern states as a beef catt'e breeding field. The price beef is now selling for and the assurance by conditions that prices will continue more steady and as favorable and in all probability become more favorable under improved methods of feeding make it logical to predict beef production in the south is only in its infancy. Many able authorities have already stated the south will eventual'y be the great beef producing section of this country and will need all the surplus pure-bred cattle the combelt can produce.

Every indication points toward greater activity and success in beef production, not only on the high priced land of the cornbe't, but also in the southern states and ranges of the west.

Beef production has been a success for centuries in Great Britain on all kinds of land, thus there is every reason to believe it can be made successful in a much larger measure and scale in this country.

The population of this country is constantly on the increase and the demand for beef becomes greater every year. The constant change from careless, slipshod methods of farming to a systematic, intensive form presents a very favorable condition for the Aberdeen-Angus breed because it not on'y thrives under rather adverse conditions, but responds most favorably to those intensive and ideal. Every condition is ripe for an unprecedented revival in the beef cattle business, and the foregoing pages set forth the overwhelming evidence the Aberdeen-Angus breed has in its favor and the manner and degree in which it leads all other breeds in the production of high-class beef, thus it behooves every Aberdeen-Angus breeder or advocate to exploit the superior merits of the famous market toppers (Aberdeen-Angus) to every cattleman and farmer in the country. 


\title{
Results of British Fat Stock Shows
}

\author{
Held at \\ London, England \\ Birmingham, England \\ Edinburgh, Scotland \\ Aberdeen, Scolland \\ Dublin, Ireland
}




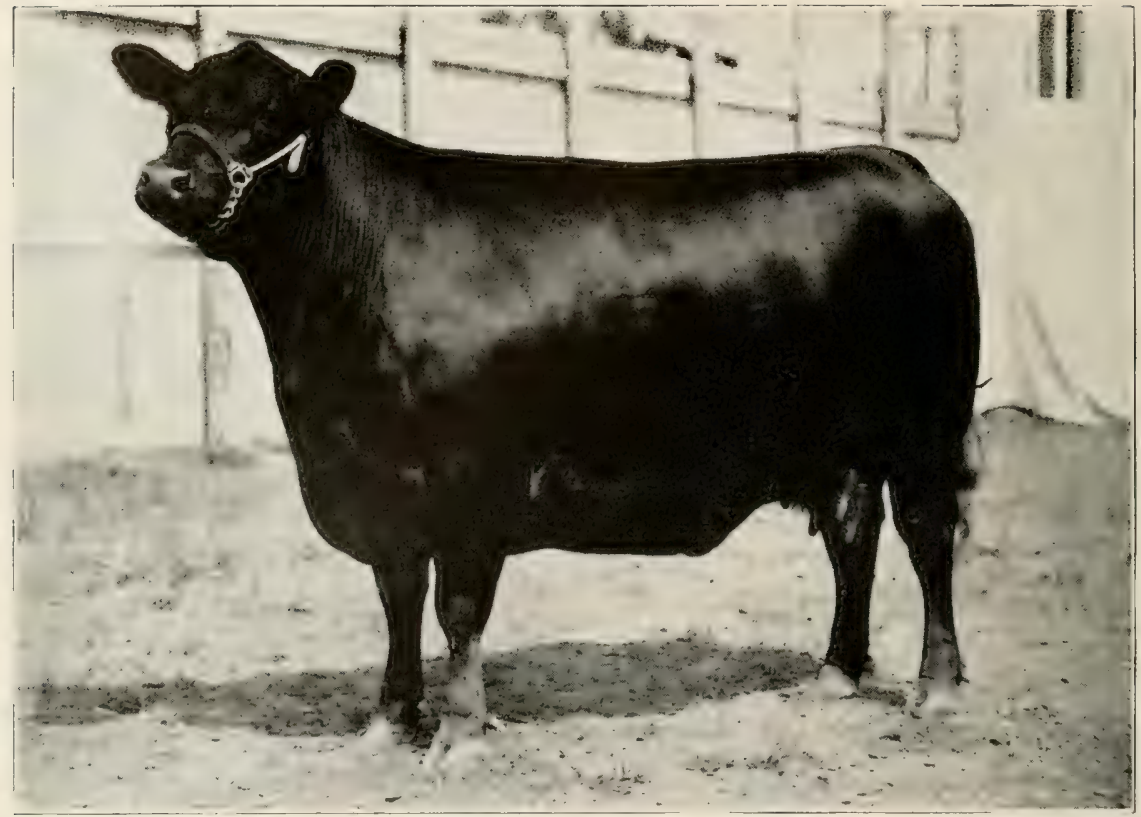

VIOLET 3d OF CONGASH.

Breeding Matron, Grand Champion Cow of Great Britain, 1909. Imported in 1910 and owned by Jas. D. NIeGregor. Grand Champion Cow of Canada and Champion Cow at International Live Stock Exposition, Chicago.

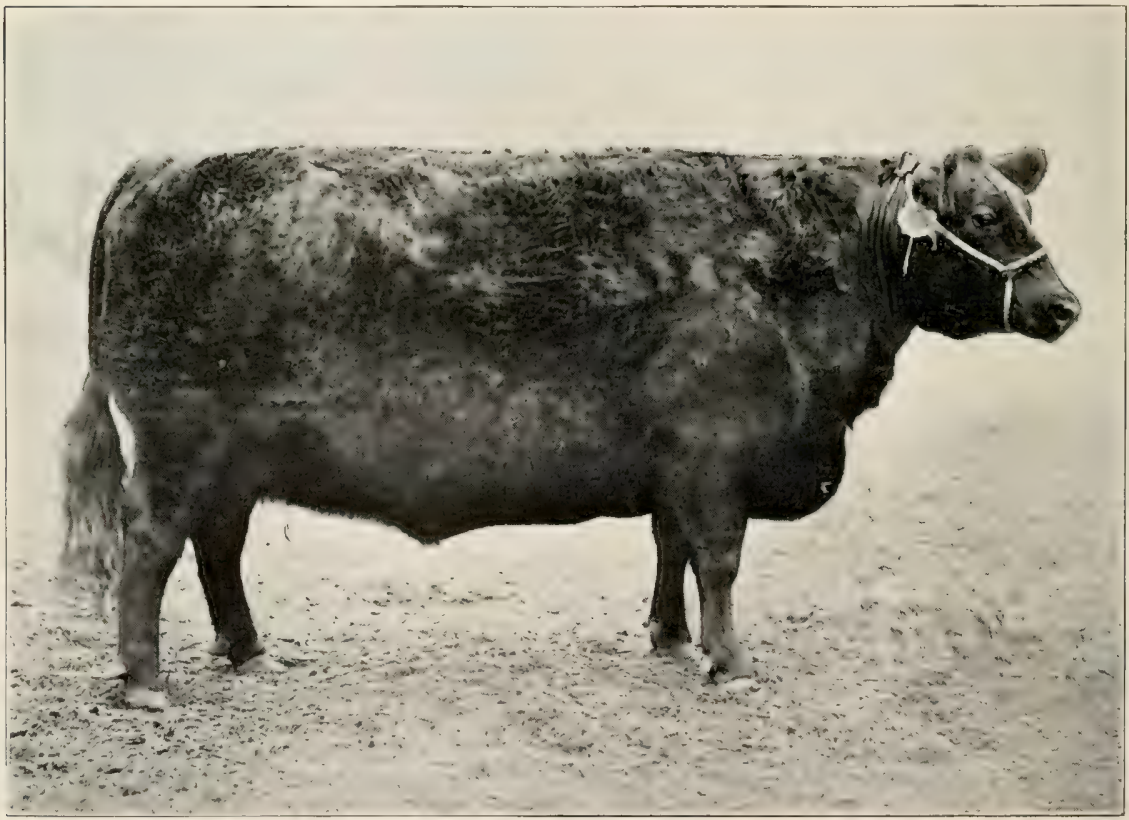

BEAUTY OF WELBECK.

1913 Grand Champion Fit Animal over all breeds at Smithfield Fat Stock Show, London, Fingland and Birmingham Fat Stock Show, Birmingham, England. Bred and exhibited by the Duke of Portland. 


\section{Introduction}

A section of this pamphlet has been devoted to the principal Fat Stock Shows of the British Isles for the purpose of presenting the results of the past decade in simple, concise and accurate form, and incidentally to show the material growth and advancement the Aberdeen-Angus breed has made in its native land.

Every American cattleman that has handled beef cattle fully realizes how much the Britons have contributed to Americans and other peoples by their prac tical and scientific work in the production of animals that have made the cattle industry of this country the greatest in the world, and one of the chief sources of America's agricultural wealth.

A study of the history of the breeds in the British Isles reveals the fact that the Shorthorn interests were stimulated at an early date and swept over, even the habitat of the Aberdeen-Angus. The Watsons, McCombie, Sir George McPherson Grant and the stanch pioneer promoters of the Aberdeen-Angus breed, gave it such an impetus about the middle and latter part of the past century, that the tide of progress of the Aberdeen-Angus breed has swept far beyond the borders of the British Isles. We must confine these brief remarks, however, to Scotland, England and Ireland. A half or even a quarter of a century ago. AberdeenAngus interests were chiefly confined to Northeast Scotland. Since the merits of the breed have become universally known it has forged its way into the South of England, leaving, as it were, a path of permanent black beasts the entire length and breadth of the United Kingdom. There has also been a steady migration of Aberdeen-Angus to Ireland. This keen demand has been especially noticeable during the past decade and has great'y curtailed the operations of Americans.

The Shorthorns have been the strongest rivals the Aberdeen-Angus have had to contend with in every part of the British Isles, but after a perusal of the following tables it must be admitted the Aberdeen-Angus have outstripped the Shorthorns.

The Hereford is a conspicuous rival of the Aberden-Angus in America, but does not furnish much competition or win many prizes when pitted against the Aberdeen-Angus and its crosses in the British Isles.

In connection with British shows we also give a review of 1913 as it was published by the Banffshire Journal which, we believe, studied in connection with tables, forms a recapitulation of points that should suffice to enlighten every cattleman of the progress the beef breeds have made in Great Britain and Ireland. 


\section{Aberdeen-Angus and Their Crosses}

Review of 1913 British Fat Stock Shows.

By J. R. Barclay, Secretary.

Breeders of Aberdeen-Angus cattle have every reason to feel proud of the renewed proof which the bred has afforded during the past fat stock show season of its superior properties in regard to beef production. Scarcely a show of any importance has passed which has not seen Aberdeen-Angus animals or crosses of that breed occupying the places of honor, and to a greater degree than ever before perhaps has the cross between Aberdeen-Angus and Shorthorn lines of breeding dominated the cross-bred classes at the various shows. Both in England, in Scotland, in Ireland and at the greatest of America's fat stock shows have the superior merits of this great beef producing breed been proclaimed, for at all the leading shows of these countries pure-bred Aberdeen-Angus anima's or crosses of that breed carried off every honor for which they could compete.

The season began as usual with the fat stock show at Norwich, and here the breed and its crosses made a debut which was in keeping with the marvelous record which was to be set up during the following three weeks. At this show the question of relative breed merits is given full scope to, as the principal section is for animals of any pure breed with the exception of Red Polls. In the young steer ciass no fewer than the first four in the list were pure-bred Aberdeen-Angus. Similarly in the section open for cross-breds, steers of Aberdeen-Angus breeding and type were first and third, while in the two-year-old class the leader was a first cross, being by a Shorthorn bull and out of an Aberdeen-Angus cow. In the class for heifers of any pure or cross breed other than Red Polls a first cross of Aberdeen-Angus type was first, a pure-bred Aberdeen-Angus second and a cross from the Aberdeen-Angus third. Even more notable were the wins of the breed and its crosses in the champion awards. The best beast bred and fed in Suffolk was a black steer. The best steer in the show was a cross-bred, as was also the reserve best steer, while the best heifer in the show was a cross-breed and the reserve a pure Aberdeen-Angus. The last two also carried off the championship and the reserve championship of the show. Thus at the initial show of the series cattle of Aberdeen-Angus breeding or crosses of the breed carried off every possible award.

Next in order came the show at Horsham, and naturally the Sussex breed won on their own ground. The reserve for the championship, however, was an Aberdeen-Angus heifer, which was also the best beast in the show other than Sussex, the reserve for this honor also going to a cross-bred heifer of Aberdeen Angus type.

Much interest as usual centered round the Birmingham show, and although Scotch cattle do not now appear there in such numbers as in former years, prior 
to the institution of the Edinburgh fat stock show, the north country breed was exceedingly well represented. In the classes for cross-bred cattle Aberdeen-Angus blood predominated, leading both in the two-year-old and yearling classes for steers and the two-year-old and yearling classes for heifers, the cup for the best crossbred and the reserve going to first crosses of Aberdeen-Angus breeding. The championship of the show was won by a pure-bred Aberdeen-Angus heifer and a black-polled cross-bred was reserve, while a steer by an Aberdeen-Angus sire won the cup for the best yearling in the show.

At the Edinburgh show there were again revealed the superior qualities of the breed and its crosses in regard to the properties of beef production, for in the four classes for cross-bred cattle, containing in all thirty-nine entries, there were only three cases in which Aberdeen-Angus breeding was not represented, while in every case the leading awards went to animals largely bred to Aberdeen-Angus blood. The champion of the section was a yearling by an Aberdeen-Angus sire, while the reserve was a two-year-old by a Shorthorn sire and out of an AberdeenAngus dam, another proof of the fact that whether introduced through the sire or through the dam, the prepotency of Aberdeen-Angus blood in beef production never fails to tell. The yearling Aberdeen-Angus Shorthorn cross steer, already indicated, won the championship of the show and a pure-bred Aberdeen-Angus steer was reserve. The championship for the best heifer was also won by a pure-bred Aberdeen-Angus, the reserve being a black-polled cross-bred.

An interesting show is always held at Redhil', and even here where the breed could not be expected to be so largely represented as at some of the other centres, it managed to hold its own, the championship being carried off by an AberdeenAngus Sussex cross, while it was noted that in the course of the fat stock shows held in the adjoining county of Kent the Aberdeen-Angus had more than ever dominated the shows, some wonderfully fine Aberdeen-Angus Sussex crosses being shown.

There was a large display of well-finished cattle at the Forres show, and here again a black polled bullock won the championship, and entries of similar breeding carried off the other leading awards.

Smithfield is of course the most important show of the kind held in Great Britain and there always exists amongst the followers of the different bresds a keen and healthy rivalry to excel at this great gathering. Of recent years no breed has produced more champion winners at Smithfield than has the Aberdeen-Angus, indeed over a period of the last dozen years or so it has provided more champions than all the other breeds combined. This year's show added fresh laurels to the Smithfield record of the breed. A pure-bred Aberdeen-Angus heifer was champion of the show and a black po'led heifer by a Shorthorn sire and out of an AberdeenAngus dam was reserve champion. Then the best steer of the show had in him a good deal of Aberdeen-Angus blood, while the reserve to him was a purc-bred Aberdeen-Angus. A blue-grey by an Aberdeen-Angus bull won the championship 
for the best yearling, and a pure-bred Aberdeen-Angus was reserve. Then as regards the carcase competition the championship went for the carcase of an Aberdeen-Angus heifer, which was pronounced by butchers to be as near perfection as is ever likely to be attained. The depth of roasting meat was extraordinary and the proportion of the cheaper qualities remarkably small. The dressed carcase weight was 65.4 per cent. of dead to live weight. The reserve for the championship went to the carcase of a cross-bred bullock combining Shorthorn and AberdeenAngus blood. It is interesting to note that in the various classes for cross-bred cattle there were in all seventy entries. Of these no fewer than fifty combined Shorthorn and Aberdeen-Angus blood, while Aberdeen-Angus blood was also shown in combination with Devons, Red Lincolns, Sussex, Red Polls, \&c. Of the twenty-four money prizes, however, every one was won by animals either by Aberdeen-Angus bulls or out of Aberdeen-Angus cows, Devons and Lincoln Reds being also successfully shown in combination with Aberdeen-Angus.

That the breeding of high-class butcher's cattle from Aberdeen-Angus foundations is also being largely and successfully followed in Ireland was again shown by the successes of Aberdeen-Angus crosses at the winter show of the Royal Dublin Society. The supreme championship of the show went to an Aberdeen-Angus steer and a byre companion bred on the same lines was reserve for that honour.

At Aberdeen, the centre of perhaps the largest cattle feeding district in Scotland, the breed again gave an exceedingly good account of itself. It won the reserve championship of the show; the cup given for the best car load of eight cattle, as also reserve for that honour; the special prize for the best butcher's animal, and the reserve; and the special prize for the best heifer in the show.

Other shows may be briefly noted. At the Suffolk show the championship went to a black polled cross heifer, while at Watford the champion was an Aberdeen-Angus, and at Exeter the leading award of the show was won by an Aberdeen-Angus heifer.

Of the Aberdeen-Angus breed at the International show at Chicago it was remarked that the Aberdeen-Angus stcry was a marvellous recital of the superlative in beef production. The display of the breed outstripped any of the previous records, startling though some of them are, and all of which augurs we'l for the solution of the absorbing problem of the future of America's beef supply; the Aberdeen-Angus breed is ready to furnish the greatest common divisor. The ripe Aberdeen-Angus steer, further remarks the greatest of America's live stock journals, has no superior as a finished product. It has a vastly important role in furnishing beef for the eager markets of the world and in finishing the raw product of cornbelt farms. In a grade Aberdeen-Angus steer-Glencarnock Victor 2nd--there was found the grand champion of the show. In the carload competition the threeyear-olds, two-year-olds, and yearlings were a!l of Aberdeen-Angus breeding, and so as a matter of course was the champion carload. Then in the carcase competition the breed scored a notable victory, securing four out of the five prizes for carcases 
of two-year-olds, and the whole five prizes for yearlings. The champion steer was an Aberdeen-Angus, which gave a dressed carcase return of 66.97 per cent.

Other shows might be dealt with in further evidence of the season's successes of the Aberdeen-Angus and its crosses, but the above may suffice, for wonderful indeed is the record which it establishes. That record may be briefly epitomized as follows:-

Norwich-Best Animal bred and fed in Suffolk; Best and Reserve Best Steer; Best and Reserve Best Yearling; Champion and Reserve Champion of Show. Horsham-Reserve Champion of the Show.

Birmingham-Best Cross-bred and Reserve; Best Yearling. Champion and Reserve Champion of Show.

Scottish Nationa!-Champion Cross-bred and Reserve; Champion and Reserve Heifer; Champion and Reserve of Show.

Redhill--Champion of Show.

Forres-Champion of Show.

Smithfield-Best Steer and Reserve Best Steer; Best Yearling and Reserve Best Yearling; Champion of Show and Reserve Champion; Champion Carcase and Reserve Champion.

Dublin-Champion and Reserve Champion of Show.

Aberdeen-Champion of Show; Best Carload; Best Butcher's Animal; Best Heifer. Suffolk-Champion of Show.

Watford-Champion of Show.

Exeter-Champion of Show.

Chicago-Champion Two-year-old; Champion One-year-old; Grand Champion of the Show; Champion and Reserve Champion Carload; Champion and Reserve Champion Carcase. 


\section{Smithfield Fat Stock Show Cross-Bred Champion and Reserve Champion Awards, 1900-1913}

\section{STEER OR HEIFER.}

1900 Champion--Shorthorn-Polled-Shorthorn

1 Reserve Champion-Aberdeen-Angus-Shorthorn-Aberdeen-Angus

1901 Champion-Polled-Shorthorn-Polled

I Reserve Champion-Shorthorn--Shorthorn-Aberdeen-Angus

1902 Champion-Shorthorn-Shorthorn-Aberdeen-Angus

1902 \{ Reserve Champion-Aberdeen-Angus-Shorthorn-Aberdeen-Angus

1903 Champion-Aberdeen-Angus-Shorthorn

1903 \{ Reserve Champion-Aberdeen-Angus-Shorthorn

$1904\{$ Champion-Shorthorn-Aberdeen-Angus

1904 Reserve Champion-Shorthorn-Aberdeen-Angus-Shorthorn

1905 I Champion-Shorthorn-Aberdeen-Angus

1905 I Reserve Champion - Shorthorn-Aberdeen-Angus-Shorthorn

$1906\left\{\begin{array}{l}\text { Champion-Shorthorn-Aberdeen-Angus } \\ \text { Reserve Champion-Aberdeen-Angus-Aberdeen-Angus-Dexler }\end{array}\right.$

$1907\left\{\begin{array}{l}\text { Champion-Shorthorn-Aberdeen-Angus } \\ \text { Reserve Champion-Aberdeen-Angus-Shorthorn }\end{array}\right.$

1908 Champion-Shorthorn-Aberdeen-Angus

1909 Champion-Aberdeen-Angus-Shorthorn-Aberdeen-Angus

1909 Reserve Champion-Aberdeen-Angus-Cross

$1910\{$ Champion-Aberdeen-Angus-Aberdeen-Angus-Shorthorn

910 Reserve Champion-Aberdeen-Angus-Shorthorn-Aberdeen-Angus

1911 Champion-Aberdeen-Angus-Shorthorn-Aberdeen-Angus

1911 \{ Reserve Champion-Aberdeen-Angus-Shorthorn-Blue Cross

1912 Champion-Aberdeen-Angus-Shorthorn

$1913\left\{\begin{array}{l}\text { Champion-Shorthorn-Aberdeen-Angus } \\ \text { Reserve Champion-Shorthorn-Aberdeen-Angus }\end{array}\right.$

\section{Smithfield Grand Champion Awards}

Grand Champion Steer or Heifer BREED

1900 Hereford

1901 Aberdeen-Angus

1902 Aberdeen-Angus

1903 Cross-bred Shorthorn-Aberdeen-Angus

1904 Shorthorn

1905 Aberảeen-Angus

1906 Shorthorn

1907 Shorthorn

1908 Aberdeen-Angus

1309 Aberdecn-Angus

1910 Aberdeen-Angus

1911 Shorthorn

1912 Shorthorn

1913 Aberdeen-Angus
Reserve Grand Champion Steer or Heifer BREED

Cross-bred Shorthorn-Polled-Shorthorn

Cross-bred Polled-Shorthorn-Polled

Cross-bred Shorthorn-Shorthorn-A berdeenAngus

Aberdeen-Angus

Aberdeen-Angus

Shorthorn

Aberdeen-Angus

Aberdeen-Angus

Cross-bred Aberdeen-Angus-Shorthorn

Cross-bred Aberdeen-Angus-Shorthorn-Aberdeen-Angus

Shorthorn

Aberdeen-Angus-Shorthorn-Aberdeen-Angus

Shorthorn

Shorthorn-Aberdeen-Angus 


\section{Summary of Smithfield Fat Stock Show Awards, 1900-1913}

The Smithfield Fat Stock Show in the sphere of Fat Stock Shows can be justly called the Supreme Court, and its decisions without question represent a true index of the best that has been produced and exhibited on the British Isles. Among the stockmen of the British Isles the Smithfield Show of London holds the same position of prestige as the International Exposition of Chicago holds among the stockmen of the United States and Canada. Further comparison would not be in order at this point; however, suffice it to say the Smithfield Show has been in existence for over one hundred years and without question has the most complete detailed classification worked out for finished fat stock, considering the field it has to perform its operations, and presents the results in the most concise and methodical manner of any Show.

A review of the past fourteen Shows reveals the bare, terse facts and shows that 267 prizes were awarded, constituting 539 different combinations of blood of the different breeds, which were won as follows: Aberdeen-Angus, 232; Shorthorns 240; Galloways, 21 ; Devon, 18; Herefords, 3; Highland, 1; Dexter, 2; Ayrshire, 1; Polled, 16; Sussex, 3; Blue-Grey, 2.

In competition for Grand Championship honors, out of fourteen possible places, pure-bred Angus won seven times and a cross-bred Shorthorn-AberdeenAngus once, pure-bred Shorthorn five times, pure-bred Hereford once. Purebred Aberdeen-Angus also won four Reserve Grand Championships and five of the other Reserve winners possessed Aberdeen-Angus or polled b'ood, while a pure-bred pure-bred Shorthorn won three times and seven of the other Reserve winners possessed Shorthorn blood. These results show that the Aberdeen-Angus and Shorthorns have furnished the bulk of the prize winners with Shorthorns a trifle in the lead in open classes. To put the results in common phraseology, other breeds have simply not been in it as far as Smithfield results are concerned. It is in competition for the highest honors, Grand Championships, that the AberdeenAngus breed is distinguished and shows superiority over the Shorthorn. The decisions of the Grand Championship honors show that when the pure-bred Shorthorns and their crosses, together with other breeds, were pitted against the AberdeenAngus and their crosses, the Aberdeen-Angus have overwhelmingly triumphed in gaining supremacy over the Shorthorns, as well as alt other breeds, in the production of high-class beef. 


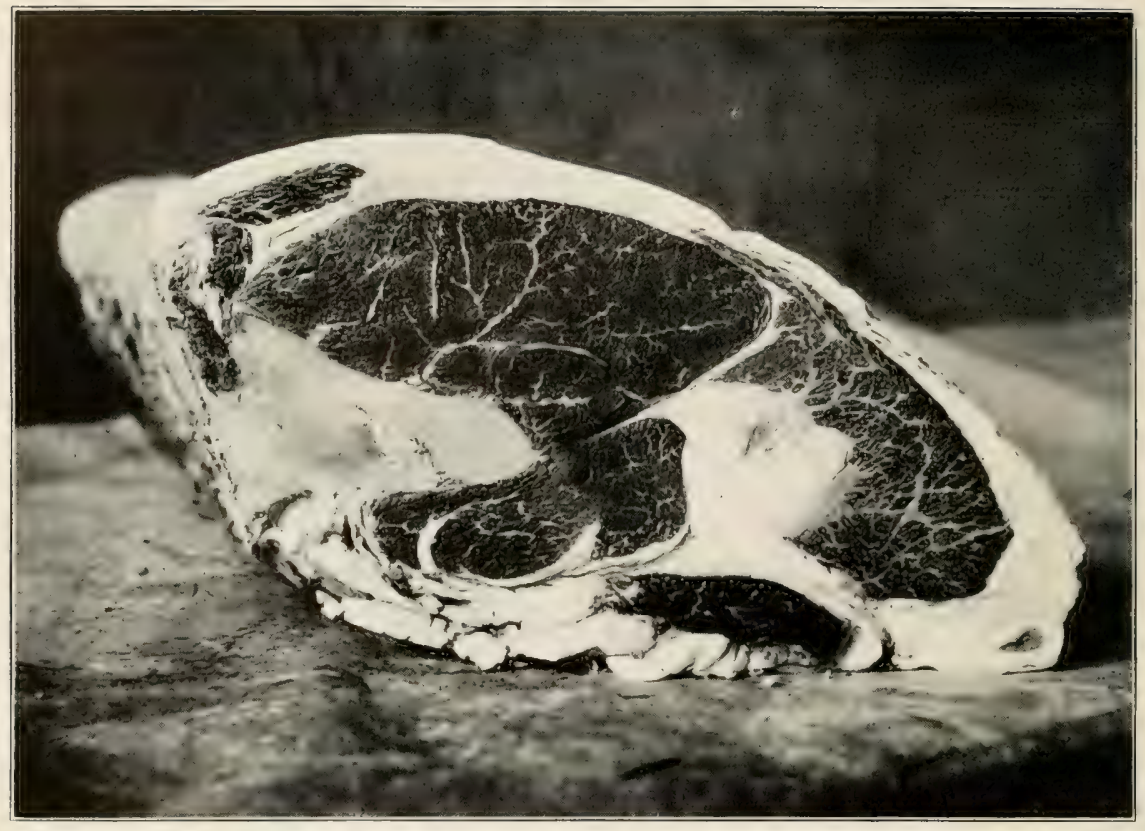

SHORT LOIN OF A GRAND CHAMHION CARCASS'.

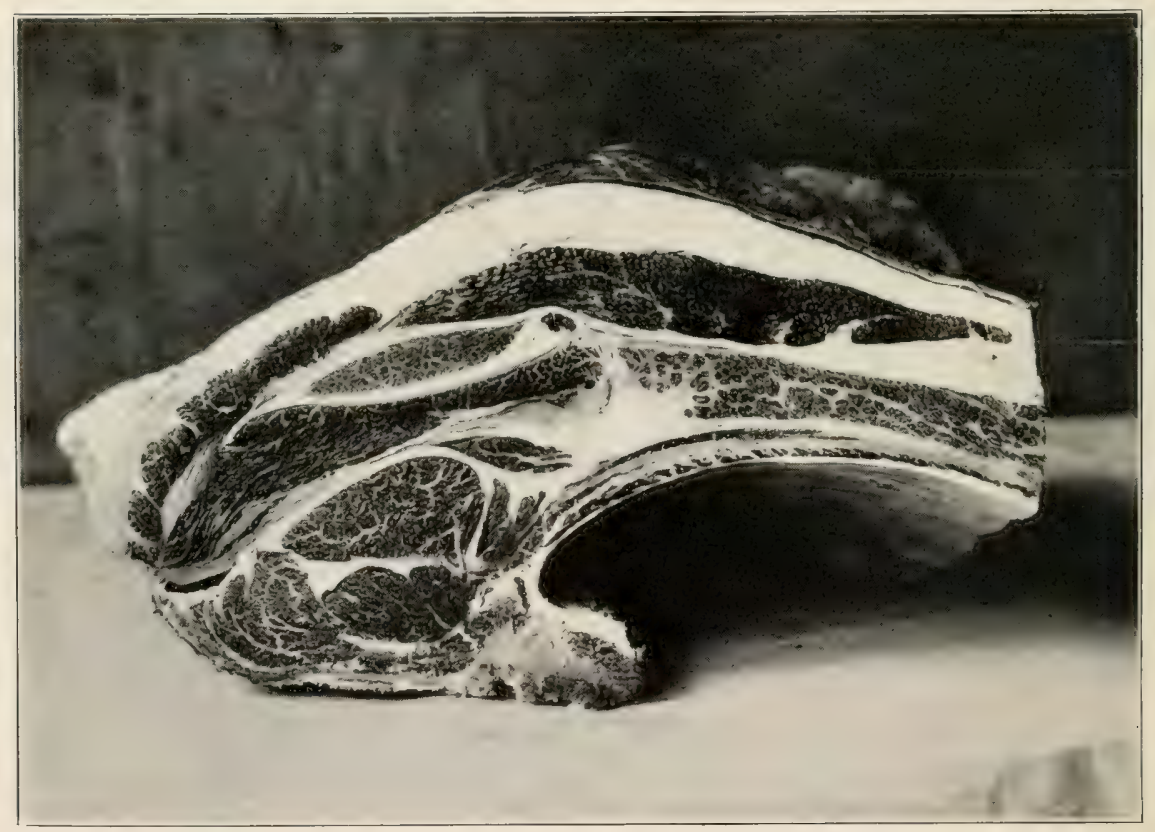

RIP ROAST OF A GRAND CHAMPION CARCASS. 




\section{Smithfield Fat Stock Show Carcass Contest Awards, 1900 - 1913}

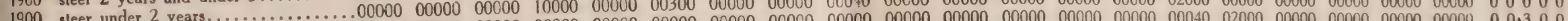
1900 heifer

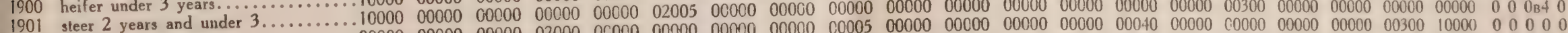

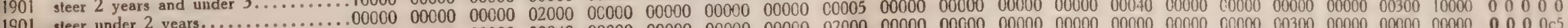

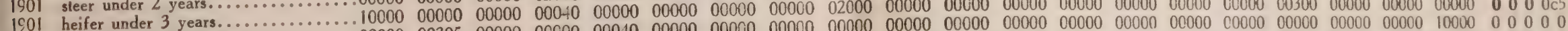

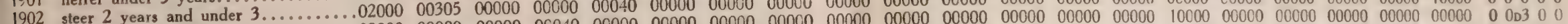

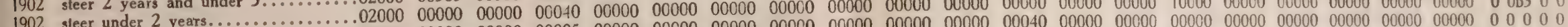

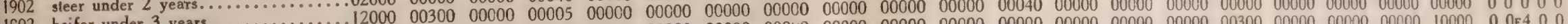
1,02 heifer under 3 (a)

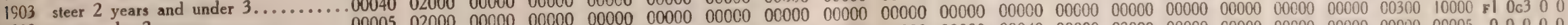
1903 steer under 2 years.................00005 02000

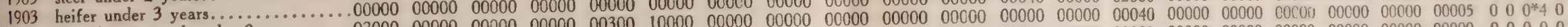

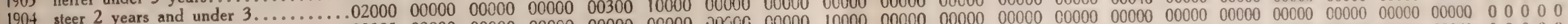
1904 ster 2 .

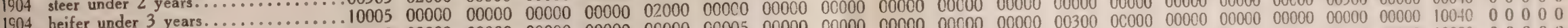

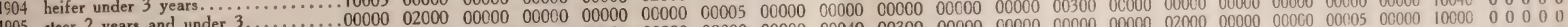

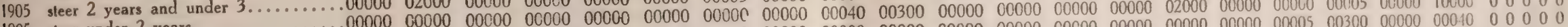
1905 steer under 2 years..................00000

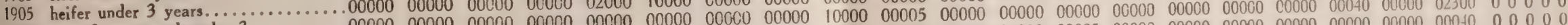

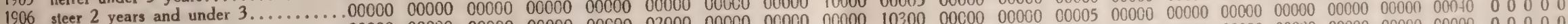
1006 steer under 2 years..................000000 $00000 \quad 00000 \quad 00000 \quad 00600 \quad 02000000000000000000001030000600 \quad 00000000000004000000000000000000000000000000000 \quad 00000$ 1906 steer under 2 years..................00000 $02000 \quad 10000 \quad 0006000300 \quad 00000 \quad 0000000000000000000000005000000000000040000000000000000000000000000002005 \quad 000000$

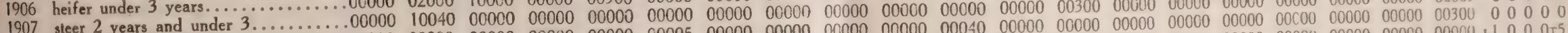

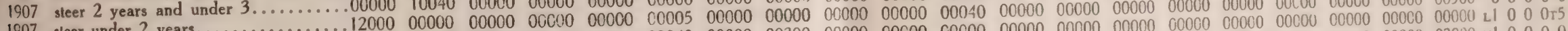
1907 sleer under 2 years..................2000 $0000000000000000000004000000003000000000000 \quad 00000 \quad 0000000000 \quad 0000000000000000000000000000000020100$ v1 0000

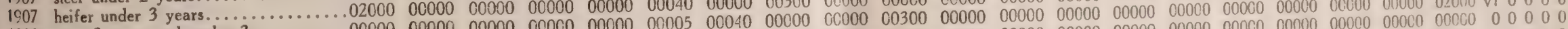

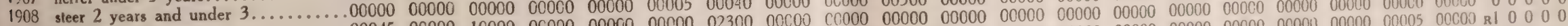

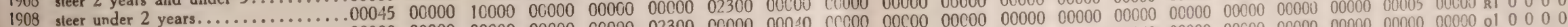

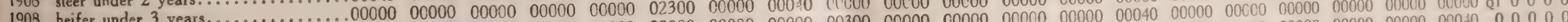

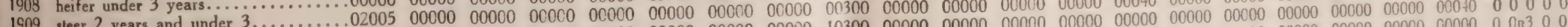
1509 steer 2 sears and

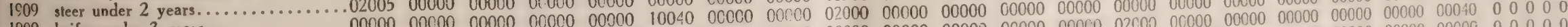

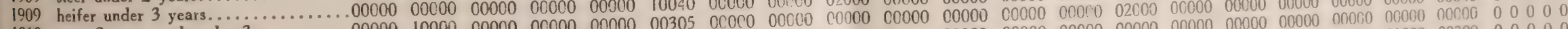

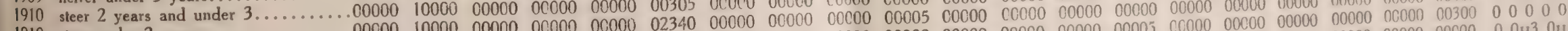

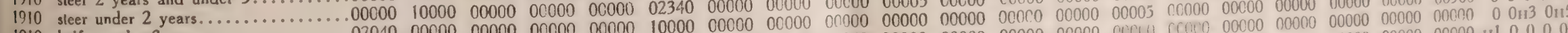
1910 heifer under 3 years. 1911 .

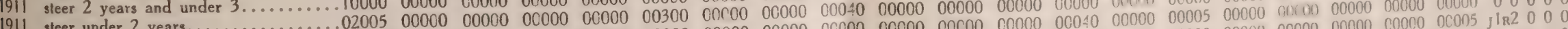

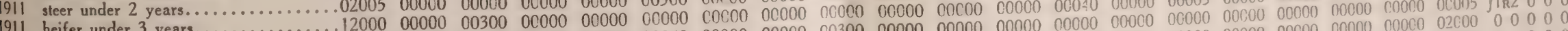

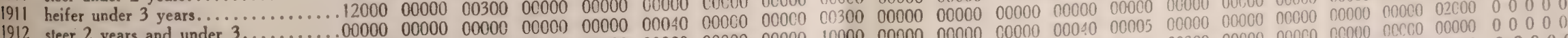

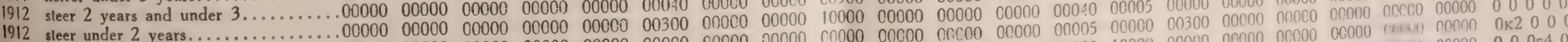

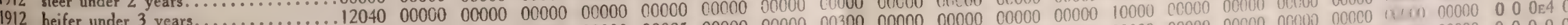

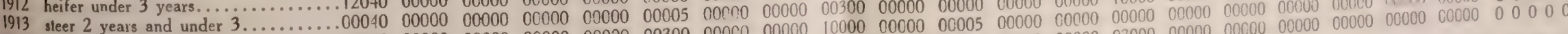

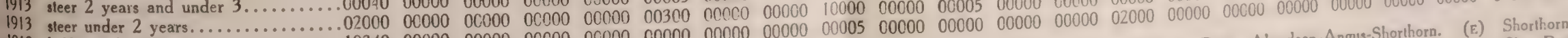

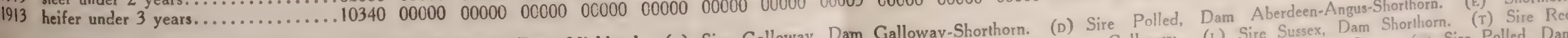

(A) Sire Aberdeen-Angus, Dam Sussex. (B) Sire Galloway, Dam Highland. (C) Sire Galloway, Dam Galloway-Shorthorn. (D) Sire Polled, (L) Sire Sussex, Dam Shorliorm Sire Polled, Darn (r) Sire Black Polled, Dam Aberdeen-Angus-Shorthorn. (c) Sire Aberdeen-Angus, Dam Shorthorn-Orkney, Highland. (o) Hire unknown, Polled, Dam Hereford. (v) Sire Galloway, Da

Highland. (x) Sire Shorthorn, Dam Highland. 



\section{Summary of Smithfield Carcass Contest}

\section{GRAND CHAMPION AWARDS}

1900 Cross-bred Aberdeen-AngusHereford.

1901 Pure-bred Aberdeen-Angus.

1902 Pure-bred Aberdeen-Angus.

1903 Pure-bred Welsh.

1904 Cross-bred Aberdeen-AngusShorthorn.

1905 Cross-bred Aberdeen-AngusShorthorn.

1906 Cross-bred Shorthorn-Aberdeen-Angus.

1907 Pure-bred Aberdeen-Angus.

1908 Cross-bred Aberdeen-AngusDexter.

1909 Cross-bred Shorthorn-Aberdeen-Angus.

1910 Cross-bred Aberdeen-AngusAberdeen-Angus Shorthorn.

1911 Pure-bred Aberdeen-Angus.

1912 Cross-bred Shorthorn-Aberdeen-Angus.

1913 Pure-bred Aberdecn-Angus.
RESERVE GRAND CHAMPION AWARDS.

1900 Cross-bred Shorthorn-Gal!oway.

1901 Pure-bred Aberdeen-Angus.

1902 Pure-bred Aberdeen-Angus.

1903 Cross-bred Black Polled Aberdeen-Angus-Shorthorn.

1904 Pure-bred Aberdeen-Angus.

1905 Pure-bred Welsh.

1906 Cross-bred Shorthorn-Aberdeen-Angus-Shorthorn.

1907 Cross-bred Sussex-Shorthorn.

1908 Cross-bred Galloway-AyrshireGalloway.

1909 Pure-bred Highland.

1910 Cross-bred Aberdeen-AngusShorthorn.

1911 Pure-bred Aberdeen-Angus.

1912 Pure-bred Aberdeen-Angus.

1913 Cross-bred Shorthorn-Aberdeen-Angus. 


\section{Summary of Smithfield Fat Stock Show Carcass Contest Awards 1900-1913}

The table of results of the Carcass Contests of the past fourteen Smithfield Shows presents facts that are worthy of consideration by every cattleman, packer and butcher.

During the fourteen years thirteen different breeds took part in the contests, and 204 prizes were awarded which consisted of 312 combinations. The number of combinations were represented by the various breeds as follows: AberdeenAngus, 127; Shorthorn, 88; Hereford, 6; Galloway, 23; Welsh, 23; Kerry, 9; Dexter, 12; Sussex, 6; Red Polled, 5; Devon, 3; Highland, 6; Pol'ed, 3; Ayrshire, 1.

In competition for Championships for fourteen years, a pure-bred AberdeenAngus won six times and seven of the other Champions possessed Aberdeen-Angus blood. One Championship was won by a pure-bred Welsh, the only Championship out of a possible fourteen that was won by an animal that did not possess Aberdeen-Angus blood. Five of the Champion winners possessed Shorthorn blood, one possessed Dexter blood, and another Hereford blood, and in each of the six cases there was a cross of Aberdeen-Angus blood.

Out of fourteen Reserve Championship prizes, a pure-bred Aberdeen-Angus won five times and four of the other prize winners possessed Aberdeen-Angus blood. Six of the crosses that were Reserves possessed Shorthorn blood, two of them Galloway blood, and the Ayrshire and Sussex were each represented once in form of a cross; a pure-bred Welsh won once and a pure-bred Highland once.

Considering the four leading beef breeds that are chiefly recognized in America and Great Britain from a percentage standpoint, the table shows 40.7 per cent for Aberdeen-Angus, 28.8 per cent for Shorthorns, 7.3 per cent for Galloways, 1 per cent for Herefords.

From the above deductions and a further realization of the fact that Smithfield and London are to the British Isles and Europe what Packingtown and Chicago are to the United States and America in regard to advancement in beef production and the formation of standards of quality for beef, there is but one conclusion that can be arrived at by all those of sound judgment and that is that the bullocks of other beef breeds are not in it with the Aberdeen-Angus bullocks when they reach the butcher's block, the final test of all animals bred and raised for meat. 


\title{
Birmingham Fat Stock Show Awards $1900-1913$
}

\author{
Birmingham, England
}

\section{Cross-Bred Champion Awards, Steer or Heifer}

BREED

1900 Shorthorn-Aberdeen-Angus-Shorthorn

1901 Aberdeen-Angus-Shorthorn

1902 Shorthorn-Shorthorn-Aberdeen-Angus

1903 Shorthorn-Galloway

1904 Shorthorn-Aberdeen-Angus

1905 Shorthorn-Aberdeen-Angus

1906 Aberdecn-Angus-Aberdeen-AngusDexter

1907 Shorthorn-Aberdeen-Angus

\section{Grand Champion Awards}

\section{Steer or Heifer}

\section{BREED}

1900 Pure-bred Shorthorn

1901 Pure-bred Aberdeen-Angus

1902 Pure-bred Aberdeen-Angus

1903 Pure-bred Aberdeen-Angus

1904 Pure-bred Devon

1905 Pure-bred Hereford

1906 Pure-bred Shorthorn

1907 Pure-bred Hereford

1908 Pure-bred Aberdeen-Angus

1909 Pure-bred Aberdeen-Angus

1910 Pure-bred Shorthorn

1911 Pure-bred Shorthorn

1912 Pure-bred Shorthorn

1913 Pure-bred Aberdeen-Angus
BREED

1909 Aberdeen-Angus-Aberdeen-Angus Shorthorn

1909 Aberdeen-Angus-Shorthorn-AberdeenAngus

1910 Shorthorn-Aberdeen-Angus

1911 Aberdeen-Angus-Shorthorn-AberdeenAngus

1912 Aberdeen-Angus-Shorthorn

1913 Shorthorn-Aberdeen-Angus

\section{Reserve Grand Champion Awards Steer or Heifer}

BREED

Pure-bred Aberdeen-Angus

Pure-bred Hereford

Cross-bred Aberdeen-Angus-Dexter

Cross-bred Shorthorn-Galloway

Cross-bred Shorthorn-Aberdeen-Angus

Pure-bred Shorthorn

Cross-bred Aberdeen-Angus-Dexter

Cross-bred Shorthorn-Aberdeen-Angus

Cross-bred Aberdeen-Angus-Shorthorn

Cross-bred Aberdeen-Angus-ShorthornAberdeen-Angus

Pure-bred $A$ berdeen-Angus

Cross-bred $A$ berdeen-Angus-ShorthornAberdeen-Angus

Cross-bred Aberdeen-Angus-Shorthorn

Cross-bred Shorthorn-Aberdeen-Angus 


\section{Summary of Birmingham Fat Stock Show Awards, 1900-1913}

The location of the Birmingham Show and the strongholds of the AberdeenAngus, Shorthorn and Hereford cattle should be borne in mind when making an analysis of the awards. Birmingham is located in the heart of England, at the very threshold of the Hereford breed and in a district where Shorthorns have been very popular for a long period of time, while the Aberdeen-Angus stronghold is located in the extreme northeast part of Scotland, thus the distance is comparatively great, and no doubt tends to reduce the number of Aberdeen-Angus entries. The chief reason, however, why the Scotch Aberdeen-Angus breeders are unable to attend the Birmingham show in greater numbers is because the Scottish National Fat Stock Show at Edinburgh, Scotland, is held the same week.

In spite of these features that have militated against the Aberdeen-Angus the past fourteen years, analysis show that out of one hundred and sixty awards constituting three hundred and fourteen combinations of blood of different breeds, the Aberdeen-Angus won one hundred and thirty-six, Shorthorns one hundred and forty-six, Galloways twenty-one, Dexters four, Devon two, Blue-grey two, Ayrshire one, Grey Polled one, Herefords only one.

Out of fourteen possible Grand Championshps (competition open to PureBreds and Cross-Breds) pure-bred Aberdeen-Angus have won six times, pure-bred Herefords twice, pure-bred Shorthorns, five times and a Devon once. Out of fourteen possible Reserve Grand Championships pure-bred Aberdeen-Angus won twice and nine other winners possessed Aberdeen-Angus blood combined with either Shorthorn or Dexter, while the remaining three Reserves were respectively won by a pure-bred Hereford, Shorthorn and cross-bred Shorthorn-Gailoway. It also will be noticed that in addition to one of the Reserve winners being a Shorthorn, eight of the other winners possessed Shorthorn blood.

In number of points for cross-breds the Shorthorn blood leads by ten. This, however, only represents the cross-bred division of the show and can not be considered as the supreme verdict of a show that offers a classification in form of Grand Championship for the competition of pure-breds and cross-breds to determine the best products. It is in the final sift for Grand Championship and Reserve Grand Championship honors that the Aberdeen-Angus completely vindicate their claim of superiority over their rivals. 


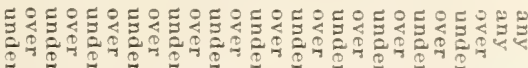

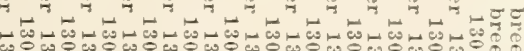
o

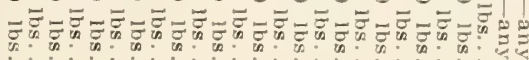

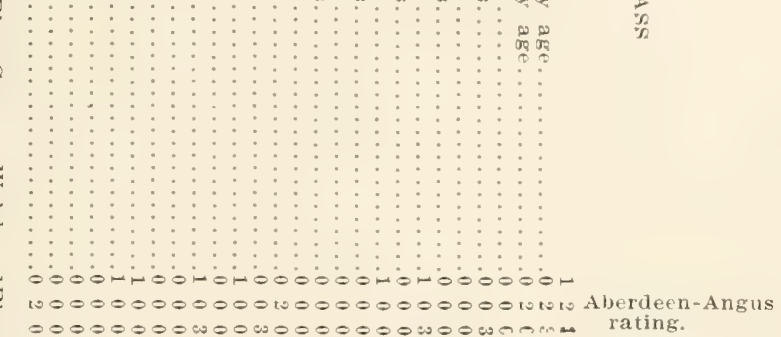

$$
\frac{2}{2}
$$

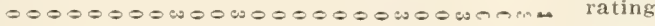

00000100000101010101000001
000000000000000000000000001

- 00000000000000000 Dam Dexter rating

opolo000000000000000000000

000000000000000000000000001 0000000000000000000010000001 000000000000001000000000000w

Aberdeen-Angus

00m00000000000000000000000w kire Aberdeen-Angus 0100000000000000101000000000010 Dam Shorthorn opupopo000000000wwopo00000u rating.

0000000000010m000000000000-1'ross-bred

0000100000100100000000000000010 Aberdeen-Angus $00000000000000000000000000 \mathrm{w}$ rating.

00000000000000000000000000- sire Galloway

00000000000000000000010000010 Dam Shorthorn 00000000000000000000000000w rating.

000000000000000000000000001

0000000000000000000000000010 Hereford rating.

$00000000000000000000 \mathrm{no00004}$

$00000000000000000000000000 \mathrm{H}$ Sire Hereford

0000000000000010000000000001 Dam Shorthern 000000000000000000000000000 rating.

000000000000000000000000001

000100000000000000010000000010 shorthorn rating. 0400000000000000001000000004

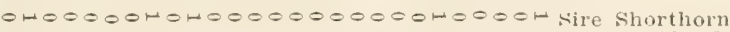
octo00100000100000000000010t0010 Dam Aberdienw0000000000000000000000000w Angus rating.

000000000000000000000000H0w Wire Shorthorn 000000000000000000000000001s Dam Galloway 000000000000000000000400000 rating.

W00Z゙0000000000 o0000010

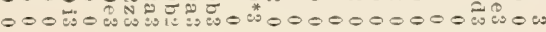




\title{
Scottish National Fat Stock Show Awards
}

\section{Edinburgh, Scotland}

\author{
Cross-Bred Animals, Steers and Heifers
}

Grand Champion and Champion Awards, 1897-1913

\begin{tabular}{|c|c|}
\hline \multicolumn{2}{|c|}{ Grand Champion Steer } \\
\hline & OR HEIFER \\
\hline & $\begin{array}{l}\text { BREED } \\
\text { BReifer }\end{array}$ \\
\hline 1898 & Shorthorn-Aberdeen- \\
\hline & Angus heifer \\
\hline 1899 & Aberdeen-Angus- \\
\hline & Shorthorn steer \\
\hline & Shorthorn heifer \\
\hline 1900 & Aberdeen-Angus steer \\
\hline 1901 & $\begin{array}{l}\text { Aberdeen-Angus- } \\
\text { Shorthorn steer }\end{array}$ \\
\hline 1902 & Shorthorn steer \\
\hline 1903 & $\begin{array}{l}\text { Aberdeen-Angus- } \\
\text { Shorthorn steer }\end{array}$ \\
\hline 1907 & $\begin{array}{c}\text { Aberdeen-Angus- } \\
\text { Cross heifer }\end{array}$ \\
\hline 1905 & Aberdeen-Angus \\
\hline & heifer \\
\hline 1906 & $\begin{array}{l}\text { Aberdeen-Angus } \\
\text { heifer }\end{array}$ \\
\hline 1907 & Aberdeen-Angus \\
\hline & Shorthorn heifer \\
\hline 1908 & Shorthorn-Aberdeen- \\
\hline & Angus heifer \\
\hline 1909 & $\begin{array}{l}\text { Aberdeen-Angus } \\
\text { heifer }\end{array}$ \\
\hline 1910 & Aberdeen-Angus \\
\hline 1911 & Aberdeen-Angus \\
\hline 1912 & Aberdeen-Angus- \\
\hline & $\begin{array}{l}\text { Aberdeen-Angus- } \\
\text { Shorthorn }\end{array}$ \\
\hline 1913 & Aberdeen-Angus- \\
\hline & \\
\hline
\end{tabular}

\section{Champion Steer} BREED

1897 Shorthorn

1898 Shorthorn

1899 Aberdeen-AngusShorthorn steer

1900 Aberdeen-AngusShorthorn steer

1901 Aberdeen-AngusShorthorn steer

1902 Shorthorn

1903 Aberdeen-Angus Shorthorn steer

1904 Highland steer

1905 Shorthorn-AberdeenAngus steer

1906 Aberdeen-AngusShorthorn steer

1907 Aberdeen-AngusShorthorn steer

1908 Aberdeen-Angus

1909 Shorthorn

1910 Aberdeen-Angus

1911 Aberdeen-Angus

1912 Gallawoy

1913 Aberdeen-AngusShorthorn
Champion Heifer or Cow BREED

1897 Aberdeen-Angus

1898 Shorthorn-AberdeenAngus heifer

1899 Aberdeen-AngusShorthorn heifer

1900 Aberdeen-Angus heifer

1901 Shorthorn heifer

1902 Aberdeen-Angus

1903 Shorthorn-AberdeenAngus heifer

1904 Aberdeen-AngusCross heifer

1905 Aberdeen-Angus heifer

1906 Aberdeen-Angus heifer

1907 Aberdeen-AngusShorthorn heifer

1908 Shorthorn-AberdeenAngus heifer

1909 Aberdeen-Angus heifer

1910 Aberdeen-Angus

1911 Aberdeen-Angus

1912 Aberdeen-AngusAberdeen-AngusShorthorn

1913 Aberdeen-Angus 


\title{
Summary of Edinburgh Fat Stock Show
}

The National Fat Stock Show held at Edinburgh is the Supreme Court, as it were, of the Fat Stock Shows held in North Britain. The foregoing shows that during a period of fourteen years one hundred and sixty-five prizes were awarded, which consisted of two hundred and ninety-seven combinations by breeds. Out of these combinations Aberdeen-Angus won one hundred and fifty-four, Shorthorns one hundred and thirty-five, Galloways five, Herefords three. From these facts it can be readily seen that in the open classes Aberdeen-Angus and Shorthorns won about equal shares of the honors, with the Aberdeen-Angus a trifle in the lead.

The list of Grand Championship honors shows that only the Aberdeen-Angus, and Shorthorns by virtue of their winnings in open classes gained entry for Championship competition. It will be further noted, however, that it is in competition for Grand Championship honors that the Aberdeen-Angus breed distinguishes itself and presents to the public in the final test an illustration of the degree in which it excels even the Scotch Shorthorns.

\section{Aberdeen Fat Stock Show Aberdeen, Scotland}

Grand Champion Awards, 1905-1909

$\begin{array}{ll} & \text { Grand Champion } \\ 1905 & \text { Aberdeen-Angus } \\ 1906 & \text { Aberdeen-Angus } \\ 1907 & \text { Aberdeen-Angus } \\ 1908 \text { Aberdeen-Angus } \\ 1909 \text { Aberdeen-Angus-Cross }\end{array}$

\author{
Reserve Grand Champion \\ Not reported \\ Aberdeen-Angus \\ Aberdeen-Angus \\ Aberdeen-Angus \\ Aberdeen-Angus
}

\section{Royal Dublin Society Show}

\author{
Dublin, Ireland
}

Grand Champion Awards, 1896-1909

1896 Cross-bred-black

1897 Cross-bred black-whiteface, sire, Aberdeen-Angus

1898 Shorthorn-Aberdeen-Angus

1899 Shorthorn-Aberdeen-Angus-blue-grey

1 c00 Polled Bullock

1901 Aberdeen-Angus

1902 Cross-bred, sire, Aberdeen-Angus

\section{BREED}

1903 Aberdeen-Angus

1904 Cross-bred blue-grey

1905 Aberdeen-Angus

1906 Aberdeen-Angus

1907 Cross-bred, Aberdeen-Angus and Shorthorn

1908 Cross-bred, dam, a cross-bred Aberdeen-Angus by a Shorthorn

1909 Cross-bred Shorthorn-Aberdeen-Angus

Detailed information of the Aberdeen Show, Scotland, and the Dublin Show, Ireland, could not be obtained. However, a sufficient number of the Grand Champion awards are given above to show that the Grand Champions of these two Shows have been Aberdeen-Angus or animals that possessed Aberdeen-Angus blood. 

LIBRARY OF CONGRESS

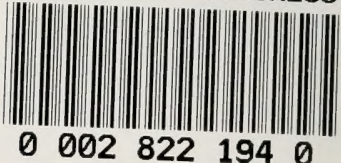

PRESS OF LIVE STOCK WJRLD

CHICAGO 